\title{
Signalling in ciliates: long- and short-range signals and molecular determinants for cellular dynamics
}

\author{
Helmut Plattner* \\ Department of Biology, University of Konstanz, PO Box M625, 78457 Konstanz, Germany
}

\begin{abstract}
In ciliates, unicellular representatives of the bikont branch of evolution, inter- and intracellular signalling pathways have been analysed mainly in Paramecium tetraurelia, Paramecium multimicronucleatum and Tetrahymena thermophila and in part also in Euplotes raikovi. Electrophysiology of ciliary activity in Paramecium spp. is a most successful example. Established signalling mechanisms include plasmalemmal ion channels, recently established intracellular $\mathrm{Ca}^{2+}$-release channels, as well as signalling by cyclic nucleotides and $\mathrm{Ca}^{2+} . \mathrm{Ca}^{2+}$-binding proteins (calmodulin, centrin) and $\mathrm{Ca}^{2+}$-activated enzymes (kinases, phosphatases) are involved. Many organelles are endowed with specific molecules cooperating in signalling for intracellular transport and targeted delivery. Among them are recently specified soluble N-ethylmaleimide-sensitive factor attachment protein receptors (SNAREs), monomeric GTPases, $\mathrm{H}^{+}$-ATPase/pump, actin, etc. Little specification is available for some key signal transducers including mechanosensitive $\mathrm{Ca}^{2+}$-channels, exocyst complexes and $\mathrm{Ca}^{2+}$-sensor proteins for vesicle-vesicle/membrane interactions. The existence of heterotrimeric G-proteins and of G-protein-coupled receptors is still under considerable debate. Serine/threonine kinases dominate by far over tyrosine kinases (some predicted by phosphoproteomic analyses). Besides short-range signalling, long-range signalling also exists, e.g. as firmly installed microtubular transport rails within epigenetically determined patterns, thus facilitating targeted vesicle delivery. By envisaging widely different phenomena of signalling and subcellular dynamics, it will be shown (i) that important pathways of signalling and cellular dynamics are established already in ciliates, (ii) that some mechanisms diverge from higher eukaryotes and (iii) that considerable uncertainties still exist about some essential aspects of signalling.
\end{abstract}

Key words: ciliates, Euplotes, Paramecium, protozoa, signalling, Tetrahymena, traffic, transport.

\section{CONTENTS}

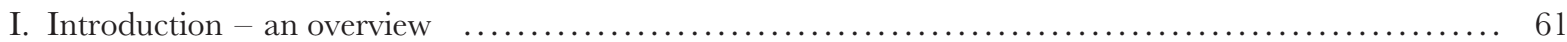

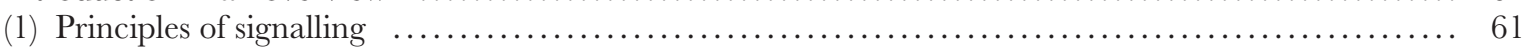

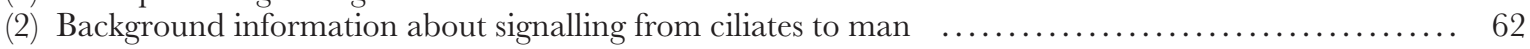

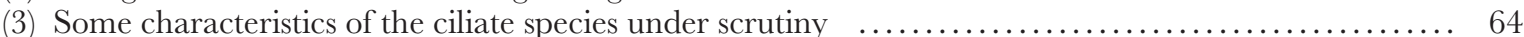

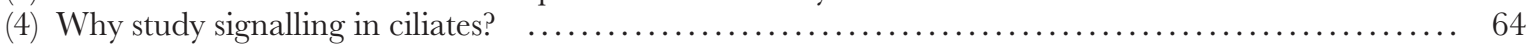

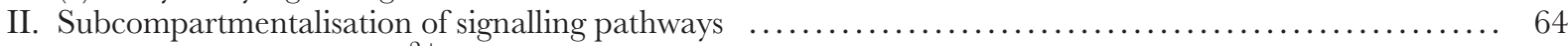

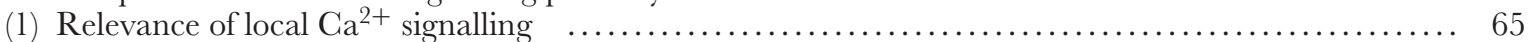

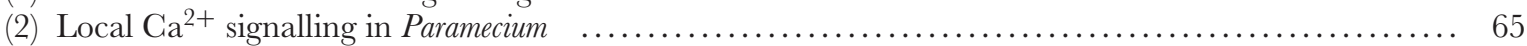

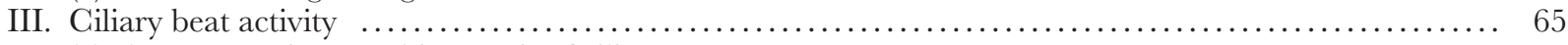

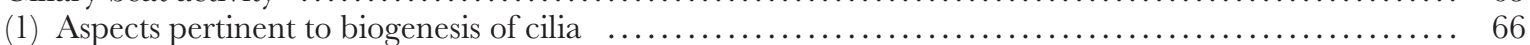

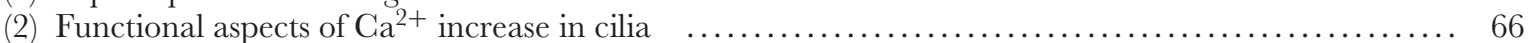

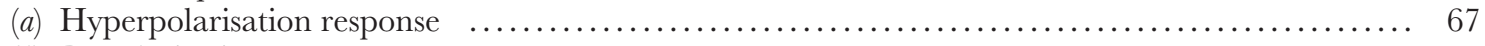

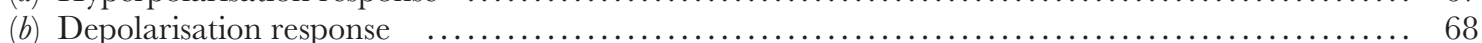

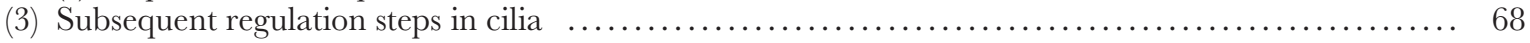

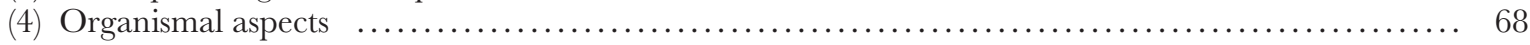

*Address for correspondence (Tel: (0)7531 88 2228; Fax: (0)7531 88 2168; E-mail: helmut.plattner@uni-konstanz.de). 
IV. Organellar trafficking signals - general rules and implications for vesicle transport in ciliates $\quad \ldots \ldots \ldots \ldots . . \ldots 9$

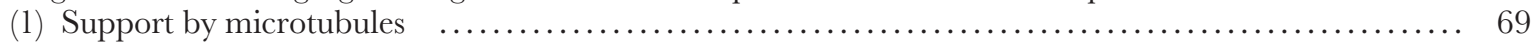

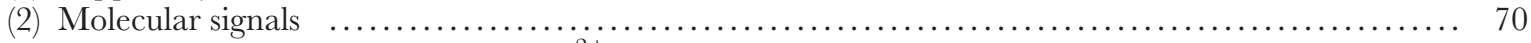

(3) Support by F-actin, SNAREs and $\mathrm{Ca}^{2+}$-dependent proteins $\ldots \ldots \ldots \ldots \ldots \ldots \ldots \ldots \ldots \ldots \ldots \ldots \ldots \ldots \ldots \ldots \ldots \ldots$

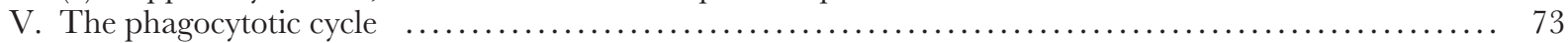

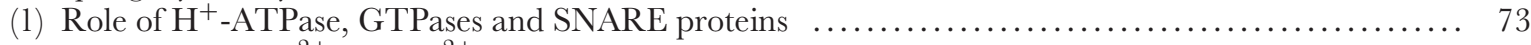

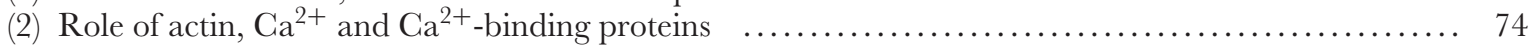

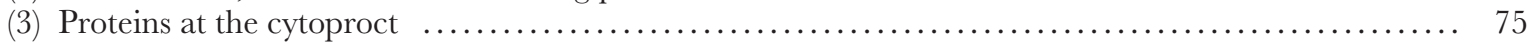

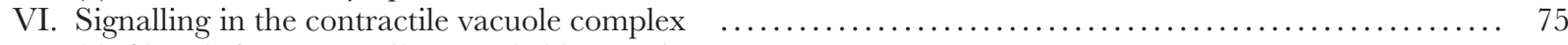

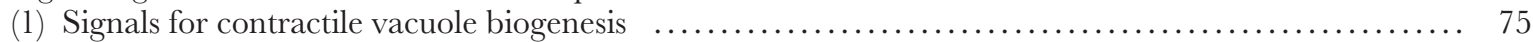

(2) Signalling in the mature contractile vacuole complex $\ldots \ldots \ldots \ldots \ldots \ldots \ldots \ldots \ldots \ldots \ldots \ldots \ldots \ldots \ldots$

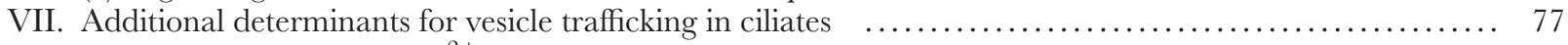

VIII. Why this multiplicity of $\mathrm{Ca}^{2+}$-release channels $(\mathrm{CRCs})$ in ciliates? $\ldots \ldots \ldots \ldots \ldots \ldots \ldots \ldots \ldots \ldots \ldots \ldots$

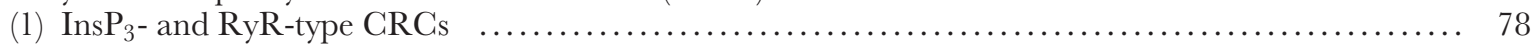

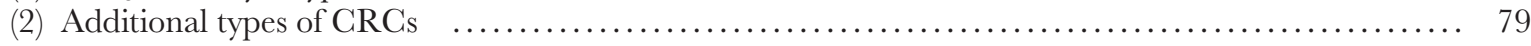

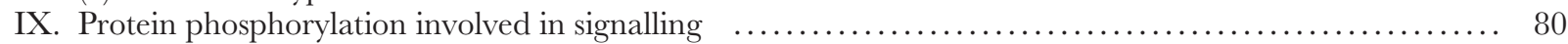

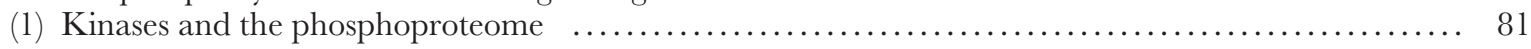

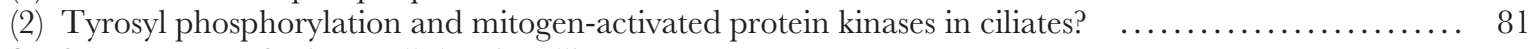

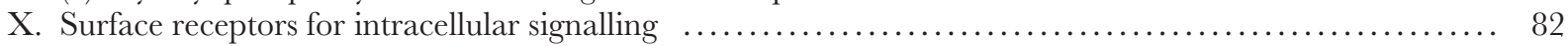

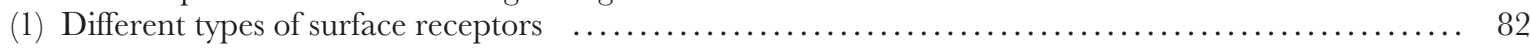

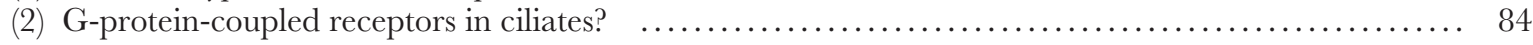

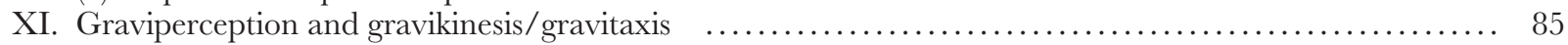

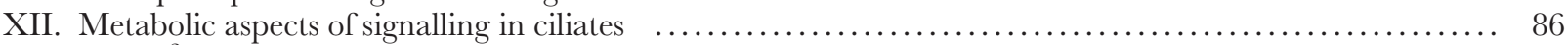

(1) $\mathrm{Ca}^{2+}$-flux, ATP decay and ATP recovery during synchronous trichocyst exocytosis $\ldots \ldots \ldots \ldots . .66$

(2) ATP decay - unlikely due to second messenger formation, SNARE rearrangement or

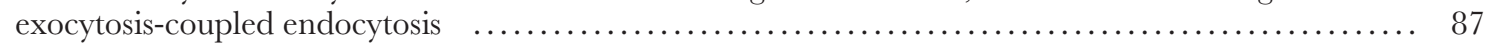

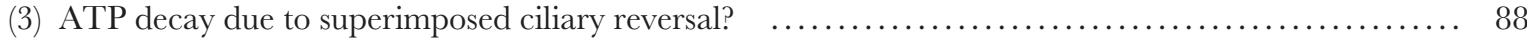

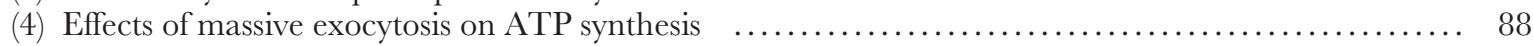

XIII. Feedback from cytoplasmic functional states, from mitochondria and from endosymbionts to the

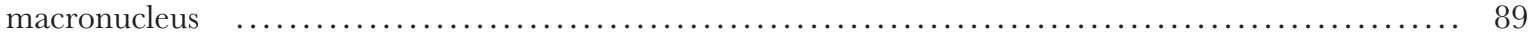

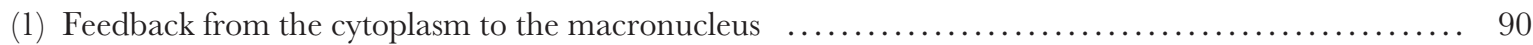

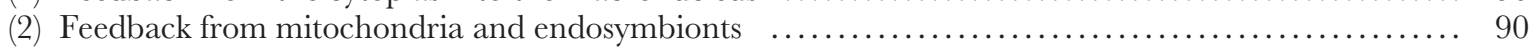

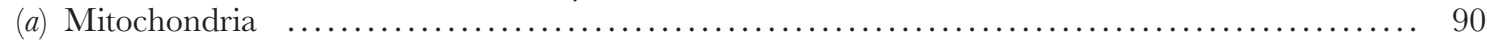

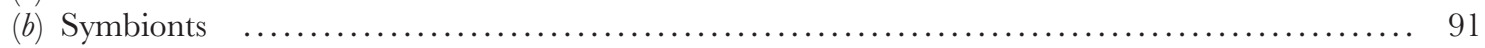

XIV. Downregulation of the most common activation mechanisms $\ldots \ldots \ldots \ldots \ldots \ldots \ldots \ldots \ldots \ldots \ldots \ldots \ldots . \ldots \ldots$

XV. Epigenetic signalling, encystment and 'programmed nuclear death' in ciliates $\ldots \ldots \ldots \ldots \ldots \ldots \ldots . \ldots . \ldots 2$

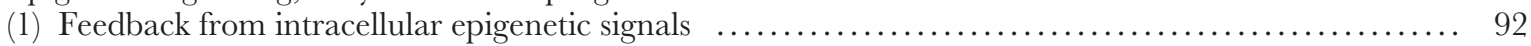

(2) Feedback from extracellular epigenetic signals, encystment and 'programmed nuclear death' $\quad \ldots . \ldots . . .93$

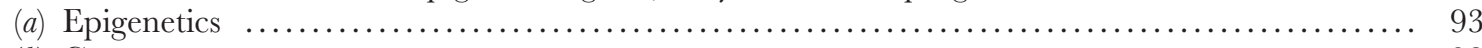

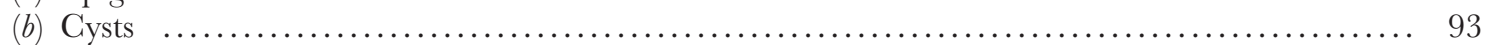

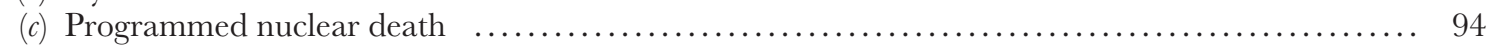

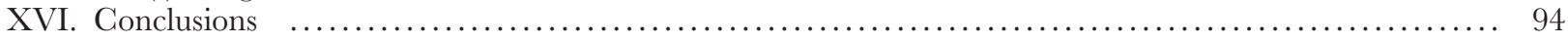

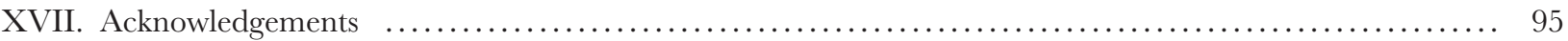

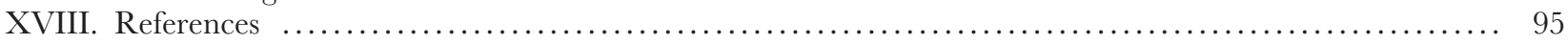

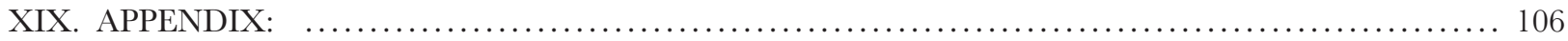

\section{INTRODUGTION - AN OVERVIEW}

\section{(1) Principles of signalling}

As cells became 'eukaryotic' by sequestration of an increased genome in a nucleus, a dialogue between the nucleus and the cytoplasm had to be organised. Within an increasing cell body, signalling became important also within the cytoplasm. Signalling implies transfer of information of widely different sorts (Good, Zalatan \& Lim, 2011). This can involve many aspects, from soluble messengers to molecular interactions of structure-bound proteins which may mediate signals via step-by-step processes. In its broadest sense, therefore, signalling includes not only primary signals and second messengers, but also molecular components on membrane surfaces which are required as short-range signals for recognition and specific organelle interaction, as well as long-range signals, such as pre-established microtubular rails for directional organelle transport (Fig. 1). Below, I outline this multiple meaning of 'signalling' and also the multiplicity of signalling mechanisms and their interactions.

Signalling involves many diverse pathways (Gomperts, Kramer \& Tatham, 2009) for which professional terminology 


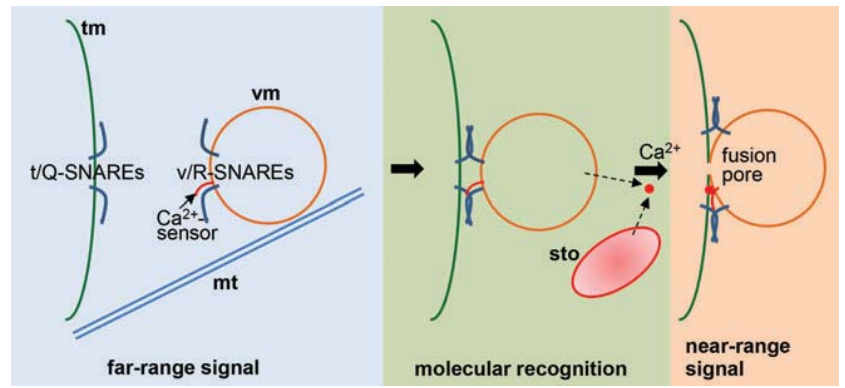

Fig. 1. Long-range and short-range signalling, and molecular cues, as exemplified by vesicle trafficking. Frequently long-range signals and short-range signals are at work consecutively, also including in defined molecular recognition. These three steps are highlighted in blue, green and red, respectively. The example given concerns the many forms of intracellular vesicle trafficking, not only in ciliates, but in man and plants. Left panel: as a long-range signal microtubular guidance helps to target organelles over a range of many micrometres to specific sites. In ciliates, this holds true, e.g. of secretory vesicle docking and of vesicle recycling processes, including guidance of vesicles to a newly forming food vacuole at the cytosome. Middle panel: at the respective target sites, within a sub-micrometre range, molecular recognition and docking of vesicles is enabled by SNARE proteins. Right panel: in a final step, molecular cues provided by SNARE proteins and associated proteins, combined with a local $\mathrm{Ca}^{2+}$ signal, act as a short-range signal within the range of tens of nanometres. Abbreviations: mt, microtubule; sto, $\mathrm{Ca}^{2+}$ store; tm, target membrane; vm, vesicle membrane. See Appendix (Section XIX) for further definitions.

and standard abbreviations are used, as summarized in a glossary in Appendix (see Section XIX). Molecular key players are $\mathrm{Ca}^{2+}$, cyclic nucleotides, protein kinases and phosphatases and a variety of target proteins.

Examples comparing ciliates with 'higher' eukaryotes are found throughout this review. A typical example of long-range signalling is the guidance of secretory vesicles by microtubular rails (see Section IV.1). The same holds for some specific recycling vesicles (see Section V.1). Cyclic nucleotides can be long- and short-range signals depending on the distance between the site of their formation and the target structure. Even $\mathrm{Ca}^{2+}$ - normally the prototype of a short-range signal (see Section II.1) - can exceptionally act as a long-range signal in spill-over phenomena (see Section II.1). Signal transfer from an endosymbiont-bearing vacuole to the macronucleus is a typical example of long-range signalling (see Section XIII.2), as is the transfer of maternal genomic information to a newly forming macronucleus in ciliates (Section XV.1). The sequence of long-range signalling and molecular recognition, followed by short-range signalling, e.g. by $\mathrm{Ca}^{2+}$, is exemplified in Fig. 1. For instance, after targetted vesicle transport along microtubules, docking occurs by molecular recognition at a target membrane via specific docking proteins, including SNAREs (see Section IV.3). $\mathrm{Ca}^{2+}$ is the final short-range signal, essentially causing membrane fusion by a conformational change of a $\mathrm{Ca}^{2+}$-sensing protein (see Section IV.3). Thus, the transfer of a signal to a remote target can be achieved. Membrane-membrane interactions during vesicle trafficking, including exocytosis, endocytosis, recycling and phagocytosis, are of this category (see Sections IV and V). Similar mechanisms can be performed in widely different situations throughout the cell, although each with specific key players (protein paralogs).

Altogether, work with metazoan cells has established that widely different signalling components and mechanisms may contribute to intracellular signalling and organellar dynamics. Signalling may involve exogenous stimuli (receptor activation, electric activation) and formation of 'metabolic activators' derived from metabolic compounds, such as cAMP (from ATP), cGMP from GTP, cyclic adenosine diphosphoribose (cADPR) and nicotinic acid adenine dinucleotidephosphate (NAADP), formed from nicotinamide adenine dinucleotide (NAD) and nicotinamide adenine dinucleotidephosphate (NADP), respectively. It may also involve $\mathrm{Ca}^{2+}$-influx and/or release, protein phosphorylation and dephosphorylation. Such messages will finally regulate a rearrangement of molecules and the transport of organelles which finally can result in specific molecular interactions.

The temporal range of 'signalling' goes from very rapid (sub-millisecond range) to more or less slow processes (minutes, hours). Directional organelle movements and recognition by cognate proteins for docking at another organelle, eventually followed by fusion, is an example of signalling whose steps range from a rather slow (intracellular transport), medium (docking) to extremely fast speed (membrane fusion). These steps all involve molecular cues and some steps require second messengers, all of which apply to eukaryotic cells, from protozoa on.

\section{(2) Background information about signalling from ciliates to man}

All this applies to eukaryotic cells, from protozoa to mammals, although information available from protozoa is much more rare than for metazoa. Among protozoa, information comes mainly from Dictyostelium spp. (Amoebozoa), whereas data for ciliates (Alveolata) became available only relatively recently. These two taxa are unicellular representatives of the two main branches of 'higher' organisms, unikonts and bikonts [also called Amorphea and Diaphoretickes and $\mathrm{SA}[\mathrm{R}] \mathrm{P}$ (He etal., 2014)]. Unikonts are related to metazoa and bikonts to plants (Baldauf etal., 2000; Adl etal., 2005, 2012; Cavalier-Smith, 2010). Considering also the close relationship of ciliates with pathogenic Apicomplexa, such as Plasmodium (malaria-causing agent) and Toxoplasma, it now is a challenge to review for the first time comprehensively signalling mechanisms in their broadest sense in ciliates.

Analysis of signal transduction in ciliates started with pioneer work using electrophysiology (Naitoh \& Eckert, 1969; Eckert \& Brehm, 1979; Machemer, 1988a,b; Saimi \& Kung, 2002). Voltage changes by de- or hyperpolarisation 
can generate distinct signals and, thus, modulate swimming behaviour which involves $\mathrm{Ca}^{2+}$ signals (Machemer, 1988b) and generation of cyclic nucleotides (Schultz \& Klumpp, 1993; Yang et al., 1997). Thus, electrophysiology with ciliates has brought about paradigms for research up to mammalian cells. By contrast, analysis of signalling inside these cells, e.g. by mobilizing intracellular $\mathrm{Ca}^{2+}$, has lagged behind for a long time.

In all eukaryotes $\mathrm{Ca}^{2+}$ can participate in mutiple ways in cellular signalling according to the following principles. Metabolic compounds can serve as second messengers. For instance, in metazoan cells, inositol 1,4,5-trisphosphate $\left(\mathrm{InsP}_{3}\right)$ generated upon stimulation from phosphatidylinositol 4,5-bisphospate $\left(\mathrm{PIns}_{2}\right)$ is an activator of a subgroup of intracellular $\mathrm{Ca}^{2+}$-release channels, CRCs (Berridge, Bootman \& Roderick, 2003), specifically of $\mathrm{InsP}_{3}$ receptors $\left(\mathrm{IP}_{3} \mathrm{Rs}\right)$. CRCs of different types can release $\mathrm{Ca}^{2+}$ ions from different organelles. Extra- and intracellular $\mathrm{Ca}^{2+}$ sources can cooperate in some cells by coordinated activity of $\mathrm{Ca}^{2+}$ influx and CRCs, both acting in concert. Thereby $\mathrm{Ca}^{2+}$ influx can either precede or follow store depletion (Barritt, 1999; Clapham, 2007). As will be discussed, only the latter mechanism is established in ciliates (see Section VIII). $\mathrm{Ca}^{2+}$ can modulate many functions as a second messenger in a cell (Berridge, Bootman \& Lipp, 1998; Berridge etal., 2003). Additional signalling processes that use $\mathrm{Ca}^{2+}$ as a direct or indirect signal encompass formation of other second messengers and/or protein phosphorylation or dephosphorylation upon stimulation, also in ciliates.

There are many more similarities between ciliates and metazoans (see Appendix). As in some mammalian cells, $\mathrm{Ca}^{2+}$ influx in ciliates occurs in concert with mobilisation from cortical pools (alveolar sacs, compartments closely attached to the cell membrane) when exocytosis is stimulated (Plattner \& Klauke, 2001). Other organelles release $\mathrm{Ca}^{2+}$ in response to $\mathrm{InsP}_{3}$ which is formed by phospholipase $\mathrm{C}$ activity also in ciliates. In metazoans, NAADP and cADPR are additional activators of different $\mathrm{Ca}^{2+}$ stores (Lee, 2012) and there is preliminary evidence that this may also hold true for ciliates (Plattner et al., 2012). In response to hyperand depolarisation, cyclic $3^{\prime}, 5^{\prime}$-adenosine monophosphate (cAMP) and cyclic 3',5'-guanosine monophosphate (cGMP), respectively, are generated in ciliates (Schultz \& Klumpp, 1993; Yang etal., 1997). In the latter case, $\mathrm{Ca}^{2+}$ is a primary 'second messenger' provided by influx and causing cGMP formation. Formation of the metabolic second messengers depends on specific cyclases and, also in ciliates, stimulation can result in transient phosphorylation of certain proteins by specific protein kinases; also protein phosphatases may depend on $\mathrm{Ca}^{2+}$ as a second messenger. Many of the signal-producing proteins and target proteins, as well as kinases and particularly phosphatases have to be positioned at proper sites to guarantee a fast and specific response (Inagaki et al., 1994). Some are assembled, together with anchoring proteins, as signalling complexes, eventually together with their targets (Good etal., 2011;
Esseltine \& Scott, 2013). Thus, a cue for proper positioning may be a prerequisite for proper local signalling from ciliates to mammals - an aspect of paramount importance particularly also for local $\mathrm{Ca}^{2+}$ signals arising from specific $\mathrm{Ca}^{2+}$ channels. Proper positioning is also important for calcium-binding proteins (CaBPs). Among them are high-affinity/low-capacity types, such as calmodulin (CaM) or synaptotagmin. Low-affinity/high-capacity forms (calreticulin, calsequestrin) are mainly contained in calcium stores. High-affinity/low-capacity CaBPs contains either EF-hand loops or C2 domains and perform extensive conformational change after $\mathrm{Ca}^{2+}$ binding, thus transmitting information, as described below. Centrin is cytosolic and possesses domains for both functions, high- and low-affinity $\mathrm{Ca}^{2+}$ binding and, thus, can also serve as a sink for $\mathrm{Ca}^{2+}$. For details in Paramecium tetraurelia, see Plattner (2014).

One of the well-established short-range signalling mechanisms, from ciliates to man and plants, is the specific recognition of vesicles/membranes of different types by a kind of key-lock mechanism using organelle-specific proteins. This task is essentially executed by specific GTPases (monomeric G-proteins) in conjunction with SNARE proteins (Jahn \& Scheller, 2006; Jahn \& Fasshauer, 2012). This acronym is derived from 'soluble N-ethylmaleimide sensitive factor [NSF] attachment protein receptors'. Eventually this leads to membrane fusion in dependency of local intracellular $\mathrm{Ca}^{2+}$ concentration, $\left[\mathrm{Ca}^{2+}\right]_{\mathrm{i}}$. As summarised previously (Plattner, 2010b) many, although not all of the molecular key players known from mammalian cells, particularly from neuronal systems (Südhof, 2013), are known also from ciliates. Yet the $\mathrm{Ca}^{2+}$ sensor involved in membrane fusion remains enigmatic in ciliates. At the cell membrane, processes such as exo- and endocytosis require a stimulation-induced local increase in $\left[\mathrm{Ca}^{2+}\right]_{\mathrm{i}}$ also in ciliates (Plattner \& Klauke, 2001). Beyond stimulated signalling, constitutive signalling also occurs, as is the case during intracellular vesicle trafficking. This also requires local availability of $\mathrm{Ca}^{2+}$ in the nearby cytosol. Local spontaneous $\mathrm{Ca}^{2+}$ signals can be seen with fluorochromes.

Cell functions discussed herein specifically for ciliates range from ciliary beating, different organelle/membrane interactions including exo- and endocytosis and phagocytosis, graviperception, apoptosis-related phenomena as well as interaction between cells by paracrine signalling. They all are accompanied by intracellular signalling processes. In its broadest sense signalling, particularly in ciliates, also has to take into consideration epigenetic phenomena (Nowacki \& Landweber, 2009; Chalker, Meyer \& Mochizuki, 2013; Simon \& Plattner, 2014). As will be discussed, there are also controversies regarding important details of signalling in ciliates. An example is the unsettled issue of phosphorylation of tyrosine residues in proteins. There is also some uncertainty about the existence of heterotrimeric GTP-binding proteins in ciliates. 


\section{(3) Some characteristics of the ciliate species under scrutiny}

Ciliates are free-living relatives of parasitic Apicomplexa, such as Plasmodium and Toxoplasma. Ciliates and Apicomplexa both belong to the superphylum Alveolata since their cortex is paved with subplasmalemmal alveoli - flat sacs serving as $\mathrm{Ca}^{2+}$ stores. Ciliate species mainly used for signalling studies are Paramecium tetraurelia and Tetrahymena thermophila, unless indicated otherwise. $P$. tetraurelia is several times bigger $(\sim 40 \times 100 \mu \mathrm{m})$ than $\mathcal{T}$. thermophila $(\sim 25 \times 50 \mu \mathrm{m})$ and was, therefore, frequently preferred for techniques such as electrophysiology, microinjection (function repair studies, antibody and $\mathrm{Ca}^{2+}$ fluorochrome studies), subcellular fractionation etc. Both species show nuclear dimorphism as they possess a diploid germline micronucleus from which a transcriptionally active 'somatic' macronucleus is derived (Orias, Cervantes \& Hamilton, 2011; Simon \& Plattner, 2014). The micronucleus is transcriptionally inactive and serves for information storage and transfer to a new macronucleus. The macronucleus is polyploid in P. tetraurelia (Aury et al., 2006; Duret et al., 2008) where, moreover, most genes are represented by a number of paralogs originating from three rounds of whole-genome duplications (Aury etal., 2006). Such genes are also called ohnologs after geneticist Ohno (1970). When ohnologs originate from the last round of duplication, they may differ only slightly in their nucleotide sequence and, therefore, most likely serve for gene amplification. This also applies to a variey of genes/proteins involved in signalling (Plattner, 2010a,b). Due to their similarities, it is difficult (although mostly not required) to differentiate between some of the most recent ohnologs by methods such as gene silencing or antibody localisation. Example are SNARE ohnologs, as discussed in Section IV.3. Since T. thermophila does not have some of these complications (Eisen et al., 2006) it can be a favourable alternative for some problems in cell signalling.

\section{(4) Why study signalling in ciliates?}

Signalling in the eukaryotic cell is also fascinating from an evolutionary point of view. In parallel to the evolution of increasingly complex and diversified cells, many mechanisms are present already in protozoans. Nevertheless, knowledge about signalling at this level of evolution is relatively scant. The patchiness of information and remarkable recent progress enabled by molecular biology provided the motivation to summarise current knowledge about signalling in ciliates herein. Among them are species of Paramecium and Tetrahymena which have served as model systems for many aspects over decades (Simon \& Plattner, 2014). As we shall see, some protein molecules and mechanisms involved in signalling are maintained from ciliates up to human brain function where they contribute to 'wire and fire' [synaptogenesis and long-term potentiation (learning)]. As will be discussed, this includes CaM, voltage-gated $\mathrm{Ca}^{2+}$ channels and their inhibition by forming a $\mathrm{Ca}^{2+} / \mathrm{CaM}$ complex; it also includes dimeric $\mathrm{Ca}^{2+}$ - and CaM-activated phosphatase 2B (calcineurin). In this we will see specific differences to plants.

There are several reasons to study signalling in ciliates. (i) Concerning one of the most important components of signalling, $\mathrm{Ca}^{2+}$, early classical electrophysiology with ciliates (see Section III) provided basic insights (Machemer, $1988 a, b)$ some of which could be successfully extended to mammalian cells. Conversely, recent progress with multicellular organisms stimulated further work with ciliates. (ii) Ciliates offer the unique possibility to induce synchronous exocytosis and rapid total deciliation, each entailing massive de novo organelle formation, based on signalling phenomena (see Section XIII.1). This allows the study of signalling during organelle biogenesis. (iii) In ciliates, a highly regular, largely epigenetically based cell structure allows for precisely localised signalling, thus facilitating structure-function correlation (Simon \& Plattner, 2014; Plattner, 2015a). The eggcase-shaped surface of a Paramecium tetraurelia cell displays $\sim 3000$ unit fields (kinetids) with about as many cilia and docking sites for dense core-secretory organelles (trichocysts) in an alternating arrangement. In the smaller genus, Tetrahymena, mucocysts substitute for trichocysts, but the cells, although smaller, have the same regular design. (iv) Every ciliate cell contains two different types of nuclei, a germline micronucleus and a transcriptionally active macronucleus, also derived from a micronucleus (Orias et al., 2011; Sperling, 2011). During macronucleus formation, genome reshaping takes place under epigenetic control and the underlying signalling mechanism can be studied (Singh et al., 2014). This is based on epigenetic information transfer via scanning RNA (scnRNA) transcripts from the parental macronucleus to the new forming macronucleus (Nowacki, Shetty \& Landweber, 2011; Swart et al., 2014). The study of epigenetic inheritance is at the forefront of research in cell biology, and not only in ciliates. $(v)$ Ciliates are at the crossroads of two lineages, unikonts and bikonts. Ciliates, originating about $0.8-1$ billion years ago (Parfrey et al., 2011; Dorrell et al., 2013), are assigned to bikonts whose further diversification led to plants (Plattner \& Verkhratsky, 2015; Plattner, 2015b). (vi) Finally, ciliates are close relatives of some of the most pernicious apicomplexan parasites, Plasmodium and Toxoplasma, which are considerably younger than ciliates (Dorrell etal., 2013). Since little is known, e.g. about $\mathrm{Ca}^{2+}$ signalling, in the parasitic forms one may expect that comparison with free-living forms may provide seminal insights (Plattner et al., 2012).

\section{SUBCOMPARTMENTALISATION OF SIGNALLING PATHWAYS}

Local limitation of signalling restricts signals to specific targets. Amongst soluble signals, this is particularly important for $\mathrm{Ca}^{2+}$ (Neher, 1998a,b). Stimulus-evoked $\mathrm{Ca}^{2+}$ signalling can occur selectively in cilia or along non-ciliary (somatic) regions of the cell membrane, whereas constitutive local intracellular $\mathrm{Ca}^{2+}$ signalling requires organelle-specific 
$\mathrm{Ca}^{2+}$-release channels. This local regulation allows for efficient, energetically low-cost information transfer and strictly localised signalling avoids inadvertent activation of additional processes. Therefore, many proteins involved in signalling are localised to specific sites, including in ciliates.

\section{(1) Relevance of local $\mathrm{Ca}^{2+}$ signalling}

The best example of the importance of compartmentalised signalling concerns $\mathrm{Ca}^{2+} \cdot \mathrm{Ca}^{2+}$ signals are in the form of dissolved (free) $\mathrm{Ca}^{2+}$ which is rapidly removed by dilution (diffusion), binding to CaBPs, sequestration into stores and/or extrusion from the cell (Berridge et al., 2003). Importantly, the efficacy of $\mathrm{Ca}^{2+}$ signals depends on the very local value of $\left[\mathrm{Ca}^{2+}\right]_{i}$ in a superlinear function (Neher, 1998a). Therefore, $\mathrm{Ca}^{2+}$-influx and $\mathrm{Ca}^{2+}$-release channels require precise localisation with regard to a specific target (Neher, 1998b). In ciliates, depolarisation-sensitive $\mathrm{Ca}^{2+}$-influx channels are found exclusively in the ciliary membrane (Machemer \& Ogura, 1979). As is known from CRCs of the superfamily including $\mathrm{IP}_{3} \mathrm{Rs}$ and ryanodine receptors (RyRs) in P. tetraurelia, a multiplicity of different $P t$ CRCs occurs in widely different organelles (Ladenburger $\&$ Plattner, 2011). Some occur only in one organelle, others in several, and one organelle may possess one or several types of CRCs of either type $\left[\mathrm{IP}_{3} \mathrm{Rs}_{\mathrm{s}}\right.$ and $\mathrm{RyRs}$ (or RyR-like proteins, RyR-LPs)]. They may mediate $\mathrm{Ca}^{2+}$ signalling in different - largely unspecified - ways and, thus, locally govern vesicle trafficking.

\section{(2) Local $\mathrm{Ca}^{2+}$ signalling in Paramecium}

The relevance of locally restricted signalling may be exemplified by one of the best-analysed phenomena in ciliates signalling for trichocyst exocytosis. Upon stimulation, $\mathrm{Ca}^{2+}$ release from alveolar sacs initiates additional $\mathrm{Ca}^{2+}$ influx via unidentified channels in the cell membrane. This sequence is termed store-operated $\mathrm{Ca}^{2+}$ entry (SOCE) also in ciliates (Plattner \& Klauke, 2001). The influx component can be seen in ${ }^{45} \mathrm{Ca}^{2+}$ flux measurements (Kerboeuf \& Cohen, 1990). Together, the internal release and influx of $\mathrm{Ca}^{2+}$, cause a sufficient increase in $\left[\mathrm{Ca}^{2+}\right]_{\mathrm{i}}$ in the narrow subplasmalemmal space to stimulate trichocyst exocytosis. This also accelerates subsequent steps leading to exocytosis-coupled endocytosis (Plattner, Braun \& Hentschel, 1997), as in different mammalian clear and dense core-secretory vesicle systems such as pituitary (Thomas et al., 1994; Wu et al., 2014) and chromaffin cells (Artalejo et al., 1995).

A $\left[\mathrm{Ca}^{2+}\right]_{\mathrm{i}}$ increase was observed in Paramecium tetraurelia using $\mathrm{Ca}^{2+}$ fluorochromes also when the extracellular $\mathrm{Ca}^{2+}$ concentration, $\left[\mathrm{Ca}^{2+}\right]_{\mathrm{o}}$, was abruptly increased, but this did not induce trichocyst exocytosis (Erxleben etal., 1997). This supports the concept of the requirement for strictly subcompartmentalized, local $\mathrm{Ca}^{2+}$ signalling; further spillover beyond the cell cortex did not lead to exocytosis (although it can activate transcriptional activity as discussed in Section XIII).
Normally $\mathrm{Ca}^{2+}$ signals in cilia do not spill over into the soma (Husser etal., 2004). Only precisely localised $\mathrm{Ca}^{2+}$ signals produce specific responses. This concept of spatially highly restricted signalling has been assessed in great depth for neuronal transmission (Neher, 1998b). All subcompartmentalisation of intracellular $\mathrm{Ca}^{2+}$ channels in ciliates serves a similar purpose. As suggested by the distribution of CRCs, in ciliates, this principle may also apply to other trafficking organelles. One example is the phagocytotic pathway (Ladenburger \& Plattner, 2011), with its complicated input, retrieval and recycling of vesicles (Fok \& Allen, 1993). In metazoans, not only $\mathrm{Ca}^{2+}$, but also 'metabolic' second messengers and proteins involved in signalling usually follow the principle of subcompartmentalisation (Barford, 2010). This also applies to ciliates, as outlined in Section VIII.

\section{GILIARY BEAT ACTIVITY}

Some of the regulation mechanisms for ciliary activity - although not all - are similar in ciliates and mammalian cells. In mammals, cAMP, cGMP and $\mathrm{Ca}^{2+}$ have a modulatory effect, predominantly by activating specific kinases (Salathe, 2007). Activation of cAMP-activated protein kinase A (PKA) accelerates ciliary beating just as in ciliates. By contrast, the role of cGMP and cGMP-activated protein kinase $(\mathrm{PKG})$ is less well defined in mammals than in ciliates. In both systems, $\mathrm{Ca}^{2+}$ has several regulatory functions. Remarkably, in ciliates, $\mathrm{Ca}^{2+}$ influx into cilia takes place via voltage-dependent channels that do not occur in animal cilia (although such channels occur in some neurons of the differentiating and differentiated central nervous system of man). In all cells, vesicles delivering proteins to cilia do not flow to the ciliary plasma membrane, but rather fuse in the nearby 'somatic' plasma membrane from where proteins dissipate into the ciliary membrane. However, the precise way in which membrane proteins and soluble proteins move into the cilia is not yet known, especially for ciliates. Several molecular cues also regulate the biogenesis of the ciliary axoneme, as discussed in Sections IV.2 and IV.3.

In ciliates, 'mature' cilia ready for function are governed by multiple signals. Normal ciliary beat activity depends on hydromechanical coupling of the cilia, as shown in ctenophores (Tamm, 2014) as well as in ciliates (Sleigh, 2014). In ciliates, modification of beat activity can be achieved by electrophysiological signals, i.e. hyperand depolarisation, respectively (Machemer, 1988a,b). The accompanying signals (ion fluxes) arise at different sites on the cell surface and are followed by formation of the cyclic nucleotides, cAMP and cGMP, respectively (Schultz et al., 1984; Schultz \& Klumpp, 1993; Yang et al., 1997). These activate the respective protein kinases for phosphorylation of specific ciliary proteins. A $\mathrm{Ca}^{2+} / \mathrm{CaM}$ complex participates in the phosphorylation of different dynein proteins and thus affects ciliary beating, as originally shown with Tetrahymena pyriformis (Blum et al., 1980). 


\section{(1) Aspects pertinent to biogenesis of cilia}

Such signalling commences with signals for proper deposition of molecular components, also in cilia. In metazoans, most membrane proteins destined for cilia are thought to be selected at different levels from the trans-Golgi network onwards (Sung \& Leroux, 2013). They may be delivered (i) first to the periciliary membrane, (ii) to more lateral parts of the cell membrane for subsequent diffusion into the ciliary membrane, or (iii) to the ciliary pocket (Bloodgood, 2012) by recycling endosomes or other vesicles. In ciliates, the latter are clathrin-coated parasomal sacs. Concomitantly auxiliary proteins contributing to the biogenesis of cilia include coatamer proteins (COPs), adaptor proteins (APs), SNAREs, GTPases including Rab-types, dynein- and kinesin-type motor proteins, intraflagellar transport (IFT) protein complexes, Beadle-Bardet Syndrome (BBS) protein complexes (BBSome) (Valentine et al., 2012), etc. SNAREs [syntaxin (Syx3), synaptosomal associated protein of $25 \mathrm{kDa}$ size (SNAP-25)] and Rab8 are considered mandatory for the formation of a primary cilium in metazoans (Kim \& Dynlacht, 2013).

Only selective aspects of targeting are available for ciliates. In ciliates, biogenesis of cilia depends on specific centrin isoforms (Stemm-Wolf etal., 2005; Jerka-Dziadosz etal., 2013). Recently it has been shown that the establishment of ciliary planar polarity (explained in Section III.2) in meningeal ependymal cells also depends on centrin (Ying etal., 2014). BBSome subunits govern channel delivery in Paramecium spp. For instance, downregulation of subunits 7, 8 or 9 reduces the delivery of $\mathrm{Ca}^{2+}$-dependent $\mathrm{K}^{+}$ channels (serving to reverse the membrane potential after depolarisation) (Valentine etal., 2012), while this does not affect voltage-dependent $\mathrm{Ca}^{2+}$ channels (whose transport pathway thus remains open). For electrophysiological details, see Section III.2. Cilia of Paramecium spp. contain the GTPase-modulating protein Sec7, i.e. transport protein 7, a guanine nucleotide exchange factor relevant for secretion; this is a ciliary homolog of ADP [adenosine diphosphate]-ribosylation factor nucleotide site opener (ARNO), a guanyl nucleotide exchange factor (GEF) found in the cytoplasm (Nair, Guerra \& Satir, 1999). In Paramecium tetraurelia, $P t$ Syx 1 is distributed over the entire cell membrane (Kissmehl etal., 2007) and thus may serve as a receptor also for docking of vesicles destined for ciliary biogenesis. Surprisingly the v-/R-SNARE (see Appendix for terminology conventions) PtSyb10 forms a ring in the periciliary cell membrane and its silencing slows down ciliary activity (cell rotation) (Schilde et al., 2010). It should be noted that generally the designation of PtSyx subtypes is comparable with those in mammals; because of wide deviations, including an additional longin domain (see Section IV.2), this is not the case with PtSyb subtypes indicated by Arabic numbers. The presence of this v-SNARE in the target membrane may be explained by an excess of vesicle delivery. Normally parasomal sacs are assumed to serve not only for constitutive endocytosis but also for constitutive exocytosis including cell membrane biogenesis
(Flötenmeyer, Momayezi \& Plattner, 1999; Capdeville, 2000). In summary, delivery mechanisms and underlying molecular cues and signals may vary for the different ciliary components, although knowledge is restricted, and not only in ciliates.

Dileptus margaritifer possesses sensory cilia on its proboscis (cell body extension used to search for prey) and locomotor cilia on the cell body. There is an enigmatic transformation of cilia when the proboscis is dissected from the cell body; this causes transformation of microtubule singlets to doublets with dynein arms (Golinska, 1982). For cilia, this is considered the first, and still the only known transformation process (Jerka-Dziadosz \& Frankel, 2014), but the signals involved remain enigmatic.

\section{(2) Functional aspects of $\mathrm{Ca}^{2+}$ increase in cilia}

Identical orientation and spatial arrangement of cilia is a prerequisite to their functional coupling in a metachronic beat pattern and this orientation depends on 'planar cell polarity'. As demonstrated in epithelia, this type of polarity requires a newly discovered coiled-coil protein, basal body orientation factor 1 (bbofl), associated with basal bodies (Chien etal., 2013). Whether a homolog exists in ciliates remains to be analysed. In $\mathcal{T}$. thermophila, disorientation protein mutant A (DisAp) protein exerts such an effect on the organisation of ciliary arrays (Galati etal., 2014) by its regulatory effect on elongation of striated (kinodesmal) fibres emanating from ciliary basal bodies in an anterior direction. Also in ciliates, functional coupling of cilia does not require any further signals; it occurs by mere hydromechanical (colligative, adhesive) forces when cilia are appropriately spaced, i.e. in the regular, epigenetically predetermined pattern (Beisson \& Sonneborn, 1965; Frankel, 1973; Beisson \& Jerka-Dziadosz, 1999; Beisson, 2008). This depends on proper positioning of daughter basal bodies during biogenesis which requires specific centrin isoforms (Stemm-Wolf etal., 2005; Jerka-Dziadosz etal., 2013) and some other conserved centrosome/basal body-associated proteins, such as FOR20 [FOP (FGFR1 oncogene partner)-related protein of $20 \mathrm{kDa}$ ] (Aubusson-Fleury etal., 2012). In conclusion, proper morphology achieved by epigenetically determined morphogenetic signalling, based on distinct co-assembly of protein components, enables coordinated ciliary beating in ciliates. This is prerequisite to mechanical coupling between cilia.

In ciliates, ciliary beating takes place spontaneously in metachronal waves due to a phase shift between longitudinally arranged, hydrodynamically coupled cilia (Machemer, 1988a). Any change in beat direction and speed requires specific signalling (Machemer, 1988b). Important examples are accelerated forward swimming due to hyperpolarisation or reversal of ciliary beat direction ('ciliary reversal') due to depolarisation (Eckert \& Brehm, 1979; Kung \& Saimi, 1982). Similarly swimming behaviour can be governed by chemo-attractants and -repellents (Van Houten, 1998). During chemosensory transduction, distinct ion conductances are activated and, thus, yield specific 
behavioural/swimming responses (Kung \& Saimi, 1982; Saimi \& Kung, 1987; Bell et al., 2007).

In ciliates, normal ciliary beat depends on $\mathrm{Ca}^{2+}$ (Naitoh \& Kaneko, 1972), as does any change in beat activity. This includes not only beat direction (see below), but also beat frequency which, in an extracted model, depends on $\left[\mathrm{Ca}^{2+}\right]$ via a $\mathrm{Ca}^{2+} / \mathrm{CaM}$ complex (Nakaoka, Tanaka \& Oosawa, 1984). Remarkably many cation channels of ciliates, ciliary and non-ciliary ('somatic'), are regulated by $\mathrm{Ca}^{2+}$ via a $\mathrm{Ca}^{2+} / \mathrm{CaM}$ complex (Kung etal., 1992; Saimi \& Kung, 1994, 2002). During ciliary reversal induction (Fig. 2), $\left[\mathrm{Ca}^{2+}\right]$ increases rapidly by influx via voltage-dependent $\mathrm{Ca}^{2+}$ channels (Machemer \& Ogura, 1979) over the entire length of a cilium in ctenophores (Tamm, 2014) as well as in Paramecium tetraurelia (Husser et al., 2004), both systems having been analysed by widely different methods. Interestingly, in ciliates, $\mathrm{CaM}$ is distributed over the entire length of cilia (Maihle etal., 1981; Momayezi etal., 1986) where it exerts different functions. One concerns the plasma membrane $\mathrm{Ca}^{2+}$-ATPase (PMCA). PMCA was demonstrated not only in the somatic plasmalemma (Wright \& Van Houten, 1990; Elwess \& Van Houten, 1997), but also in cilia of Paramecium tetraurelia (Yano et al., 2013). This confirms older biochemical data from Tetrahymena thermophila (Dentler, 1988). PMCA may, therefore, possibly belong to the standard inventory of cilia not only in ciliates but also up to mammals. For instance, in cilia of olfactory neurons of mice, PMCA contributes to the downregulation of $\mathrm{Ca}^{2+}$ signals (Saidu et al., 2009). It should be noted, however, that no information is available for PMCA in cilia of ctenophores and that cilia in metazoa beyond ctenophores contain other types of $\mathrm{Ca}^{2+}$-influx channels.

In mammals, this pump is activated by subplasmalemmal actin (Dalghi et al., 2013). Actin also occurs in cilia of ciliates (Kissmehl etal., 2004), notably isoforms PtAct1, PtAct2, PtAct3, PtAct4, and PtAct5 (Sehring et al., 2007b). Another function at the cell membrane level is the activation of cation channels pertinent to ciliary activity (Preston et al., 1991).

How can the ciliary beat be changed? A physical signal (anterior or posterior mechanical stimulation) results in an electric signal. Hereby a receptor potential (hyperor depolarisation) is formed at the level of the somatic cell membrane, followed by an action potential formed at the level of the ciliary membranes (Kung \& Saimi, 1982). As described below, this is followed by formation of cyclic nucleotides, activation of protein kinases and phosphorylation of specific proteins. All this prepares the cilium for specific mechanical work.

\section{(a) Hyperpolarisation response}

Accelerated forward swimming by hyperpolarisation is triggered not only by touching cells at the rear end, but also by an appropriate change in extracellular ionic conditions or by adding a chemoattractant (Kung \& Saimi, 1982; Yang etal., 1997; Van Houten, 1998). The hyperpolarisation-induced receptor potential is carried by a $\mathrm{K}^{+}$outward current (Naitoh \& Eckert, 1969) and further on

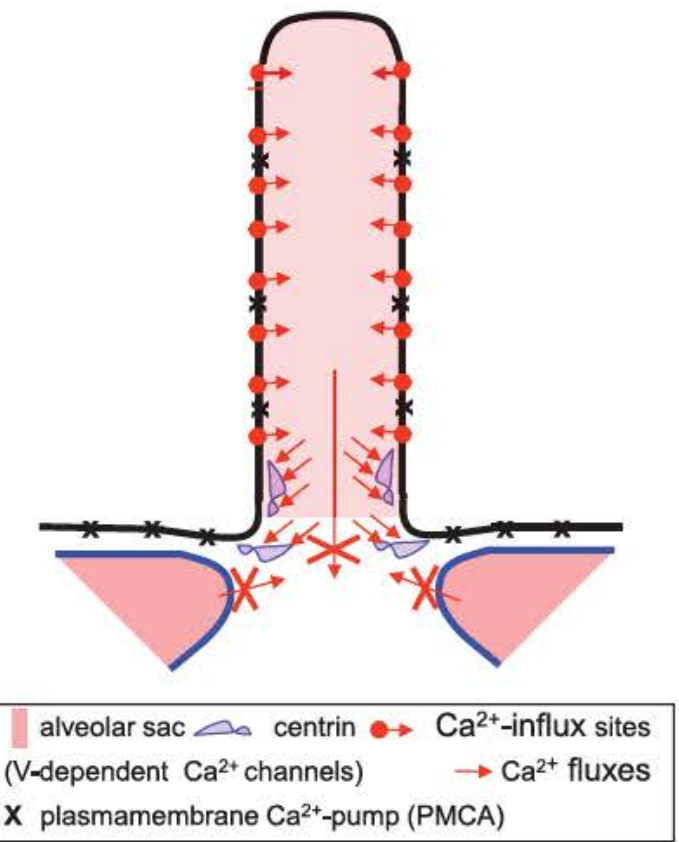

Fig. 2. Ciliary reversal is an example of spatial signal restriction in ciliates. Depolarisation activates voltage-gated $\mathrm{Ca}^{2+}$ channels. The influx of $\mathrm{Ca}^{2+}$ is restricted to several hundred ions per cilium by negative feedback via a $\mathrm{Ca}^{2+} /$ calmodulin complex (not shown). $\mathrm{Ca}^{2+}$ in cilia is downregulated by binding to $\mathrm{Ca}^{2+}$-binding proteins (CaBPs), such as centrin, within the cilium and at its base, thus avoiding signal spread into the cell soma. The occurrence of a plasma membrane $\mathrm{Ca}^{2+}$-ATPase (PMCA) in cilia is thought to be likely. Note that normally cortical calcium stores (alveolar sacs) do not contribute to this ciliary activity.

activates highly $\mathrm{Ca}^{2+}$-selective hyperpolarisation-activated channels in the somatic membrane, peaking within $20-80 \mathrm{~ms}$ (Preston, Saimi \& Kung, 1992a). This is followed by activation of adenylyl cyclase which forms cyclic AMP (cAMP), a process depending on the hyperpolarising $\mathrm{K}^{+}$ conductance (Bonini, Gustin \& Nelson, 1986; Schultz \& Schönborn, 1994). According to quenched-flow analyses cAMP is formed already within one ciliary stroke (Yang etal., 1997) which lasts $\sim 40 \mathrm{~ms}$. [Note that the minimal analysis time for quenched-flow analyses includes the $\sim 30 \mathrm{~ms}$ dead time of the instrument]. Thus, there is a perfect match between an electrical and a chemical signal. This cyclase, that is reportedly $\mathrm{Ca}^{2+}$ sensitive (Kudo etal., 1985a; Kudo, Muto \& Nozawa, 1985b), is an integral membrane protein coupled to an ion channel domain with a voltage sensor and a $\mathrm{K}^{+}$-pore loop (Weber et al., 2004). Subsequent activation of a PKA in cilia (Hochstrasser \& Nelson, 1989) causes phosphorylation of specific ciliary proteins, such as components of dynein, the motor protein responsible for ciliary movement (Hamasaki etal., 1991; Walczak \& Nelson, 1993; Kutomi et al., 2012). This chain of events accelerates forward swimming by accelerated dynein activity, before the increased cortical $\left[\mathrm{Ca}^{2+}\right]_{\mathrm{i}}$ inactivates the hyperpolarisation-sensitive $\mathrm{Ca}^{2+}$-influx channels (Preston, 
Saimi \& Kung, 1992b); any participation of CaM in the inactivation of the hyperpolarisation response has not been investigated as yet.

\section{(b) Depolarisation response}

Ciliary reversal is induced not only by mechanical stimulation at the anterior part of the cell, e.g. when a cell hits an obstacle, but also by an appropriate change of ionic conditions in the medium, such as increased $\left[\mathrm{K}^{+}\right]_{\mathrm{o}}$ causing depolarisation. In the first case, mechanosensitive channels enriched at the anterior part of the somatic cell membrane are activated (Ogura \& Machemer, 1980). A receptor potential forms by a $\mathrm{Ca}^{2+}$ influx (Naitoh \& Eckert, 1969), followed by a $\mathrm{K}^{+}$ efflux (Satow, Murphy \& Kung, 1983), both in the somatic (non-ciliary) part of the cell membrane (Machemer, 1988a,b). This triggers a $\mathrm{Ca}^{2+}$-based action potential by activating depolarisation-activated (voltage-dependent) $\mathrm{Ca}^{2+}$ channels that are restricted to the ciliary membrane (Machemer \& Ogura, 1979) (Fig. 2). The membrane potential is returned to resting level by a voltage-gated and $\mathrm{Ca}^{2+}$-activated $\mathrm{K}^{+}$ current - the respective channels also residing in the cilia (Valentine et al., 2012). Most of these data have been collected from Paramecium tetraurelia. Remarkably, voltage-gated $\mathrm{Ca}^{2+}$ channels are also found in our central nervous system.

Depolarisation entails formation of cGMP (Schultz, Pohl \& Klumpp, 1986), by activation of $\mathrm{Ca}^{2+} / \mathrm{CaM}$-activated guanylate cyclase (Kudo et al., 1985a,b; Schultz \& Klumpp, 1991) and of a PKG, all in cilia (Miglietta \& Nelson, 1988). The respective guanylate cyclase is localised to the cilia membrane (Linder et al., 1999). Phosphorylation of a set of proteins somehow causes the reversal of the ciliary beat direction. Inhibition of phosphatase 1 (PP1), sustains backward swimming (Klumpp, Cohen \& Schultz, 1990), thus reflecting a role in signal termination. $\mathrm{Ca}^{2+}$ influx is short since voltage-dependent $\mathrm{Ca}^{2+}$ channels are inactivated by the same $\mathrm{Ca}^{2+}$ that they conducted, by forming an inhibitory $\mathrm{Ca}^{2+} / \mathrm{CaM}$ complex (Brehm \& Eckert, 1978). Again the same mechanism is maintained up to the mammalian central nervous system (Levitan, 1999). Here, an additional $\mathrm{Ca}^{2+}$-binding protein, $\mathrm{CaBP} 1$, and the $\mathrm{Ca}^{2+} / \mathrm{CaM}$ complex mutually control each other (Findeisen, Rumpf \& Minor, 2013). It remains to be seen whether a similar fine-tuning mechanism also exists in ciliates.

\section{(3) Subsequent regulation steps in cilia}

Anywhere in the cell, free $\mathrm{Ca}^{2+}$ can be rapidly downregulated by binding to CaBPs. Total calcium (structure-bound + dissolved) and free (dissolved) calcium can be differentially determined by using energy-dispersive $\mathrm{X}$-ray microanalysis (EDX) and fluorochrome analysis, respectively. In Paramecium spp., this has been exemplified not only with exocytosis (see Section IV) but also with cilia (Husser et al., 2004). Upon depolarisation, an increase of total and free calcium can be detected in the cilia within one ciliary beat. As seen by EDX, total calcium concentration remains at an increased level, in contrast to free $\left[\mathrm{Ca}^{2+}\right]$ which rapidly decays according to fluorochrome measurements. This indicates that $\mathrm{Ca}^{2+}$ ions are rapidly bound and trapped inside cilia. Nevertheless, the reversal reaction lasts tens of seconds before normal forward swimming is resumed. Remarkably, this is much longer than the signal of free $\mathrm{Ca}^{2+}$ (Husser et al., 2004). The ongoing response may be sustained by the occurrence of additional $\mathrm{Ca}^{2+} / \mathrm{CaM}$ complexes in the axoneme where CaM also occurs (Momayezi et al., 1986). The actual reversal reaction has tentatively been explained by occupancy of the carboxy-terminal $\mathrm{Ca}^{2+}$-binding loops of CaM by $\mathrm{Mg}^{2+}$ (Machemer \& Teunis, 1996) - the last of the four hierarchically occupied EF-hand $\mathrm{Ca}^{2+}$-binding loops. Moreover, in cilia differential binding of $\mathrm{Ca}^{2+}$ to other $\mathrm{Ca}^{2+}$-dependent and $\mathrm{Ca}^{2+}$-independent $\mathrm{CaM}$ binding proteins (CaM-BPs) takes place (Evans \& Nelson, 1989), although their respective roles are not precisely known. Not only PKA, PKG and CaM, but also $\mathrm{Ca}^{2+}$-dependent protein kinases (CDPKs), which possess an integrated CaM-like sequence (see Section IX.1), are found in the cilia of Paramecium tetraurelia (Kim, Messinger \& Nelson, 1998). Collectively these mechanisms can prolong ciliary reaction in some way beyond the actual duration of the free $\mathrm{Ca}^{2+}$ signal in cilia.

Thus, a physical signal (hyperpolarisation or depolarisation) results in $\mathrm{Ca}^{2+}$ signal, then in a chemical signal (cyclic nucleotides) which results in the covalent modification (phosphorylation) of ciliary proteins. The spectrum of proteins phosphorylated by PKA and by PKG, respectively, is different (Ann \& Nelson, 1995) and they govern ciliary motility in different ways (Bonini \& Nelson, 1990). These mechanisms transmit mechanical stimuli to chemical signals which in turn are transmitted to a mechanical response, i.e. accelerated forward swimming or ciliary reversal. Thus, $\mathrm{Ca}^{2+}$ can initiate several ciliary responses along different pathways (Machemer \& Teunis, 1996) and this is achieved by modification of the phosphorylation state of specific proteins.

\section{(4) Organismal aspects}

During ciliary reversal, normally no $\mathrm{Ca}^{2+}$ is spilled into the soma of a Paramecium tetraurelia cell (Husser et al., 2004). A spill-over in the reverse direction does occur, however, when massive exocytosis is induced. Then, $\mathrm{Ca}^{2+}$ moves from the cell cortex into cilia and, thus, induces ciliary reversal without previous depolarisation (Husser etal., 2004). This also entails cGMP formation (Knoll, Kerboeuf \& Plattner, 1992) and, thus, this process proceeds as during ciliary reversal induced by depolarisation. Such automatic coupling of trichocyst exocytosis and ciliary reversal is also triggered when a Paramecium tetraurelia cell contacts a predatory cell (Knoll, Haacke-bell \& Plattner, 1991b); this is meaningful in the context of the escape mechanism that helps to ward off predators such as Dileptus margaritifer (Harumoto \& Miyake, 1991). Currently such escape phenomena are known in an increasing number of ciliates (Buonanno, Harumoto \& Ortenzi, 2013). During this defence reaction, whose primary chemical signal is unknown, vigorous local trichocyst release first forms a spacer between predator and prey, while 
the prey activates the 'reverse gear' (Knoll et al., 1991b). This is perfectly mimicked by the standard secretagogue for Paramecium spp., aminoethyldextrane (AED). Its local application induces local trichocyst release also in deciliated cells as well as in mutants devoid of voltage-dependent $\mathrm{Ca}^{2+}$ channels (Plattner et al., 1984; Plattner, Stürzl \& Matt, 1985). In such cells AED also rapidly induces ciliary reversal by spillover of $\mathrm{Ca}^{2+}$ from the soma into cilia as documented by fast confocal fluorochrome and EDX analysis (Husser etal., 2004). Since this reaction is short it may easily be overlooked (Harumoto, 1994). Thereby the depolarisation step is bypassed. Lectins contained in the trichocyst tip (Haacke-Bell \& Plattner, 1987) may additionally contribute to the defence mechanism.

\section{ORGANELLAR TRAFFICKING SIGNALS - GENERAL RULES AND IMPLICATIONS FOR VESICLE TRANSPORT IN CILIATES}

In mammalian cells, the principal pathway of vesicles from the endoplasmic reticulum to the cell membrane was elucidated by Palade (1975) and the underlying molecular machinery by Rothman (2014). The basic principles of trafficking also apply to ciliates.

Long-range guidance of vesicles during trafficking along microtubules has been detected in mammalian gland cells (Lacy etal., 1968) and motoneurons (Gray, 1978). In these cells, as in most others, transport goes from the minus to the plus end of microtubules, i.e. from the cytocentre to the cell periphery. Transport in the reverse direction was first described in Paramecium tetraurelia (Plattner, Westphal \& Tiggemann, 1982), subsequently in mammalian epithelial cells and finally in immune cells (Griffiths, Tsun \& Stinchcombe, 2010). Short-range signals and target proteins for final signal transduction are quite similar in protozoa and metazoa. Such chemical signals inherent to proteins govern the direction of vesicle trafficking, attachment and fusion. A paradigmatic example is neurotransmitter release (Südhof, 2013, 2014).

Short-range signals encompass several proteins mediating vesicle docking and fusion, with the involvement of $\mathrm{Ca}^{2+}$, SNARE (Rothman, 2014; Sühof, 2014) and Rab proteins (Mizuno-Yamasaki, Rivera-Molina \& Novick, 2012; Pfeffer, 2013), as specified below. This was detected with mammalian cells and yeast before being explored in ciliates (Bright etal., 2010; Plattner, 2010a,b). These basic aspects are early evolutionary achievements maintained throughout eukaryotic kingdoms (Dacks \& Field, 2007; Kloepper, Kienle \& Fasshauer, 2008; Stenmark, 2012).

This section contains multiple facets of signalling phenomena as exemplified in Figs 1, 3 and 4 for the secretory pathway and the phagocytotic cycle in Paramecium. Surprisingly trichocysts contain signals for their filling state, for their inherent polarity and, thus, for their docking competence. Short-range signals or molecular cues, as known from metazoans, encompass vesicle- (v-) SNAREs

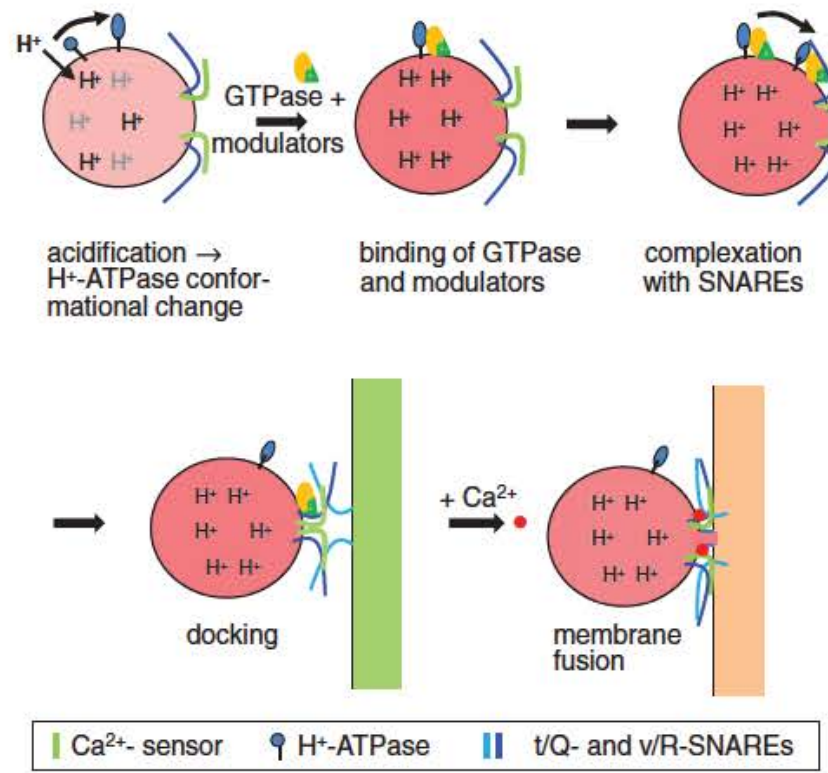

Fig. 3. Molecular cues and short-range signals governing membrane trafficking in ciliates reside in SNARE proteins, small GTPases (Rab proteins) and their modulators, in addition to $\mathrm{H}^{+}$-ATPase molecules. In mammalian cells, luminal acidification has been shown to cause a transmembrane signal by a conformational change in the multimeric $\mathrm{H}^{+}$-ATPase molecule, thus enabling the binding of Rab proteins via their modulators and then forming a complex with SNAREs. For membrane fusion to occur as the final signalling step $\mathrm{Ca}^{2+}$ has to be provided as a short-range signal.

which, together with additional proteins, have to match with appropriate target- (t-)SNAREs (Jahn \& Fasshauer, 2012) (Fig. 3). This also applies to ciliates (Plattner, 2010a,b) (Fig. 4). Upon a local increase of $\left[\mathrm{Ca}^{2+}\right]_{\mathrm{i}}$ during the course of stimulation both membranes can fuse. $\mathrm{Ca}^{2+}$ flushing from the outside medium through the exocytotic opening into the trichocyst lumen provides an additional signal, required specifically for the explosive decondensation and vigorous release of trichocyst contents (Plattner \& Klauke, 2001).

\section{(1) Support by microtubules}

Trichocyst transport is saltatory (Aufderheide, 1978), i.e. facilitated and guided by microtubules emanating from ciliary basal bodies, along which they slide in minus-end direction for final docking at the cell membrane(Plattner et al., 1982). The regular arrangement of docking sites between regularly spaced cilia in Tetrahymena thermophila and Paramecium tetraurelia is mediated by epigenetic signals (Frankel, 1973; Beisson, 2008). Organelles undergoing trafficking encompass not only dense core-secretory organelles, i.e. trichocysts in Paramecium spp. and mucocysts in Tetrahymena spp., but also clear vesicles for the biogenesis of the plasmalemma (Schilde et al., 2010) and release of acid hydrolases (Kiy et al., 1993) at ill-defined sites. In ciliates, recycling vesicles and phagosomes also travel along defined routes determined by microtubular arrays (Fok \& Allen, 1993; Wloga \& Frankel, 2012). In 


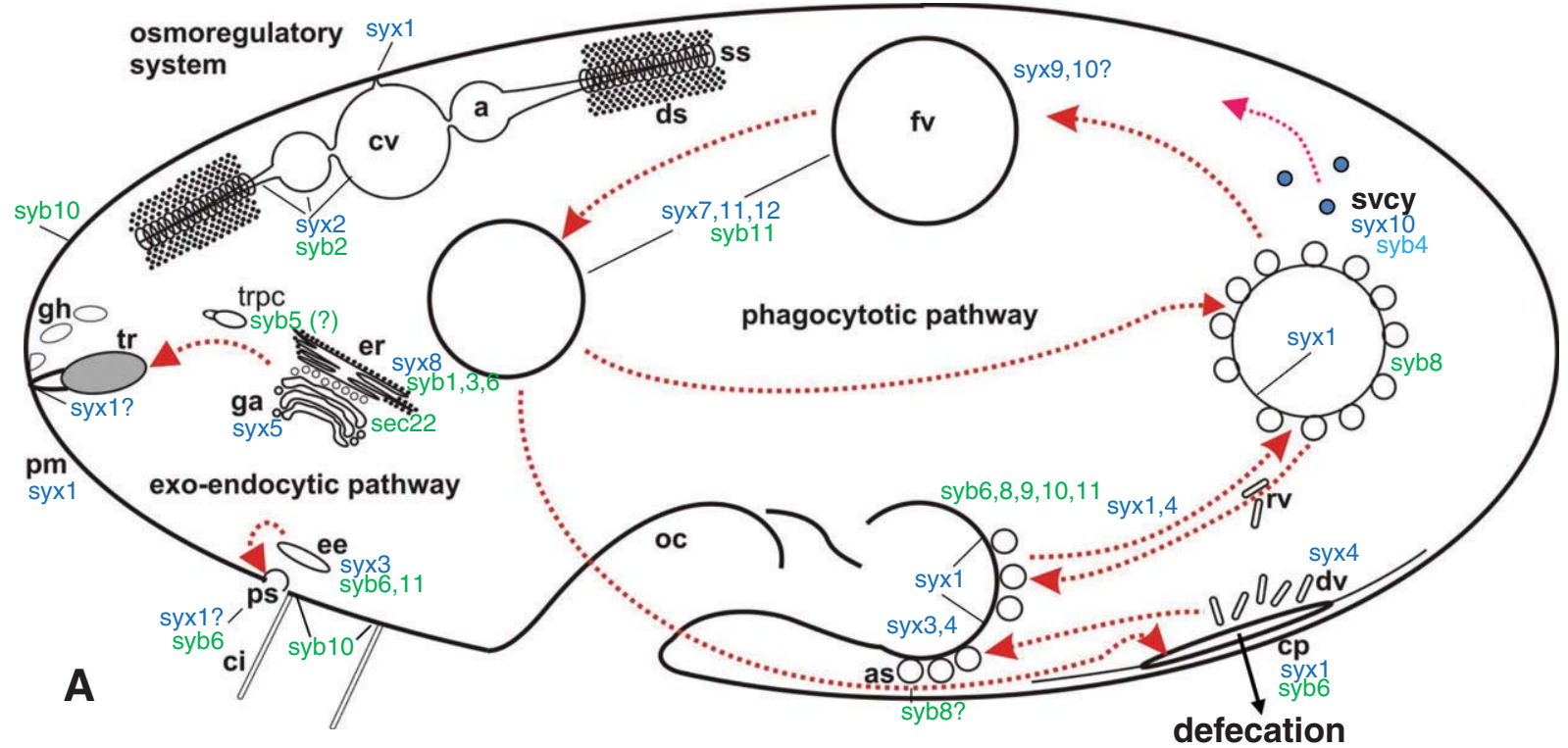

Fig. 4. The membranes of vesicles trafficking along the principal pathways in a Paramecium sp. cell (nuclei omitted) contain specific proteins, such as SNAREs. Trafficking routes of the phagocytotic cycle are indicated by dotted red arrows. Subcellular distribution of isoforms of the t-/Q-type SNARE, syntaxin (syx), and of the v-/R-type SNARE, synaptobrevin (syb), are compared. Abbreviations: $\mathrm{a}=$ ampullae (on contractile vacuole); $\mathrm{as}=$ acidosomes (late endosomes); $\mathrm{ci}=$ cilium; $\mathrm{cp}=\mathrm{cytoproct}$; $\mathrm{cv}=\mathrm{contractile}$ vacuole; $\mathrm{ds}=$ decorated spongiome (part of contractile vacuole complex); $\mathrm{dv}=$ discoidal vesicles (recycling from cytoproct); ee $=$ early endosomes, $\mathrm{er}=$ endoplasmic reticulum; $\mathrm{fv}=$ food vacuole; ga $=$ Golgi apparatus; $\mathrm{gh}=$ ghost (empty trichocyst membrane); oc $=$ oral cavity; pm = plasma membrane; $\mathrm{ps}=$ parasomal sacs (clathrin-coated endocytotic vesicles); $r \mathrm{v}=$ recycling vesicles; $\mathrm{sec} 22=$ transport protein 7 (guanine nucleotide exchange factor) relevant for secretion; ss = smooth spongiome (part of contractile vacuole complex); svcy $=$ small vesicles in cortical cytoplasm; $\operatorname{tr}=$ trichocyst; $\operatorname{trpc}=$ trichocyst precursor.

T. thermophila microtubules have been shown to contain $\alpha$-tubulin in association with a variety of different $\beta$-tubulins, thus allowing for differential functionalisation (Pucciarelli et al., 2012). Furthermore, a number of microtubules are composed of distinct post-translational modifications of tubulin, in Paramecium tetraurelia (Adoutte et al., 1991) as well as in Tetrahymena thermophila (Wloga etal., 2008; Wloga \& Gaertig, 2010). Such arrays compose the '(post)oral fibres' in Paramecium multimicronucleatum and P. tetraurelia or the 'deep fibre' in Tetrahymena thermophila; they guide nascent food vacuoles and drive recycling vesicles back to the cytopharynx (Allen, 1974).

\section{(2) Molecular signals}

In mammalian cells, the lumen of all these travelling organelles is more or less acidic due to their endowment with a V-type $\mathrm{H}^{+}$-ATPase (Mellman, 1992; Forgac, 2007). Also they store more or less $\mathrm{Ca}^{2+}$ inside (see Section VIII). It was also shown with mammalian cells that acidification causes a conformational change in the heterooligomeric $\mathrm{H}^{+}$-ATPase molecule which, thus, transmits a signal to the cytoplasmic side. This in turn allows for the docking of small GTPase molecules and their respective modulators (Hurtado-Lorenzo etal., 2006). Rab-type GTPases mediate directional transport, for instance in the exo- and endocytotic pathway by interacting with organelle-specific protein effector molecules.
In higher eukaryotes, the different types of trafficking organelles contain specific GTPases and their respective effector molecules as well as complementary SNAREs on each of the interacting membranes. GTPases are activated by binding GTP which causes a conformational change. Minor changes in amino acids in GTPases can change their specific binding capabilities, whereas effectors usually have no common binding motif (Grosshans, Ortiz \& Novick, 2006). Some SNAREs have been recognised as effector partners (Zerial \& McBride, 2001). This also applies to longin-type v-SNAREs, variations of 'synaptobrevins' containing a longin domain, for some of which binding of small GTPases has been shown (De Franceschi etal., 2014). Although still called 'synaptobrevins' longin-type SNAREs are the predominant v-/R-type SNAREs in plants and in ciliates such as Paramecium tetraurelia (Schilde etal., 2006, 2010) and Tetrahymena thermophila (De Franceschi et al., 2014). Globally this means that in eukaryotes specificity of membrane interaction is determined by several cues.

An example of an effector molecule is early endosome antigen 1 (EEAl), the marker of the early endosomal membrane of metazoan cells (Mishra et al., 2010). (Unfortunately such organelle-specific proteins interacting with GTPases are practically unknown from ciliates.) As mentioned, GTPases contain structural determinants providing interaction with specific effectors (Khan \& Ménétrey, 2013). Such proteins enable reversible binding to, and reversible activation of 
the next compartment of the trafficking pathway (Pfeffer, 2013). Modulatory proteins include GTPase activating protein (GAP), guanosine nucleotide dissociation inhibitor (GDI) and guanosine nucleotide exchange factor (GEF). Together with organelle-resident SNAREs, firmly installed by a single-pass carboxy-terminal stretch, this complex is the key for docking at the specific target membrane. Finally, in concert with a discrete $\mathrm{Ca}^{2+}$ signal and a $\mathrm{Ca}^{2+}$-sensor protein (Rizo, Chen \& Arac, 2006), a trans-complex of vand t-SNAREs can mediate fusion (Jahn \& Scheller, 2006). As established in metazoans, this type of signal recognition by molecular cues is essential for delivery of vesicles to their correct targets by matching protein-protein interaction. What is the situation in ciliates?

$\mathrm{H}^{+}$-ATPase (Fok et al., 2002; Wassmer et al., 2005, 2006) and SNAREs (Plattner, 2010b) are documented for all trafficking vesicles in $P$. tetraurelia, together with small GTPases in T. thermophila (Bright etal., 2010). GTPase modulating proteins GAP, GDI and GEF in ciliates await elucidation. Although the $\mathrm{Ca}^{2+}$ content of trafficking vesicles is unknown in ciliates, its presence can be inferred on the one hand from the presence of $\mathrm{Ca}^{2+}$ in such organelles in mammalian cells (Section VIII.2) and on the other hand from the presence of CRGs in these organelles in Paramecium spp. (Ladenburger \& Plattner, 2011).

The situation during the secretory cycle in ciliates is as follows. Dense core-secretory vesicles of ciliates, such as trichocysts (Paramecium spp.) and mucocysts (Tetrahymena spp.), collectively called extrusomes in the different ciliate species, contain in part similar and in part different contents (Verbsky \& Turkewitz, 1998) and they serve different purposes (Hausmann, 1978). Trichocysts are bipolar structures, with a 'tip' and a 'body'. The presence of SNAREs in the $P$. tetraurelia trichocyst membrane, probably $P t$ Syb5, is most likely, although not identified conclusively (Schilde etal., 2010). Extrusomes are special in several ways. Trichocysts contain no recognisable $\mathrm{H}^{+}$-ATPase (Wassmer etal., 2005, 2006) and the organelles are not recognisably acidic (Lumpert etal., 1992; Wassmer etal., 2009), including trichocyst precursor stages (Garreau de Loubresse, Gautier \& Sperling, 1994). No $\mathrm{Ca}^{2+}$ is detected inside trichocysts (Hardt \& Plattner, 2000). However, low numbers of $\mathrm{H}^{+}$-ATPase and low concentrations of $\mathrm{Ca}^{2+}$ cannot be excluded. Remarkably a sufficient level of cytosolic $\mathrm{Ca}^{2+}$ is required for trichocyst biogenesis (Ladenburger et al., 2009). Possibly $\mathrm{Ca}^{2+}$ has to be removed from the lumen at a certain stage of maturation since $\mathrm{Ca}^{2+}$ would cause decondensation of the contents after maturation (proteolytic processing), as it does during exocytosis when exogenous $\mathrm{Ca}^{2+}$ gets access to the organelle lumen (Bilinski, Plattner \& Matt, 1981; Klauke et al., 1998). This sequence of events, $\mathrm{Ca}^{2+}$-insensitive precursor $\rightarrow \mathrm{Ca}^{2+}$-sensitive proteolytically cleaved mature granule lattice protein (pro-Grlpl $\rightarrow$ Grlpl), has been verified in detail for Tetrahymena thermophila mucocysts (Verbsky \& Turkewitz, 1998).

In T. thermophila, lysosomal sorting receptors, homologous to sortilin of higher eukaryotes, represent a signal for the biogenesis of mucocyst contents. This includes proteins relevant for proteolytic processing of some precursors, their eventual aggregation and/or sorting in part in soluble and in part in insoluble form (Briguglio, Kumar \& Turkewitz, 2013). In detail, non-aggregating protein, granule tip protein Grtlp, a constituent of the organelle tip, is bound by a specific subtype of sortilin, in contrast to the granule lattice protein, Grl3p; the latter, however, requires a sortilin-mediated mechanism indirectly, as this selects cathepsin 3 which in turn is required for Grllp processing.

As known from mammalian cells, during maturation of dense core-secretory organelles a proprotein convertase (Steiner, 1998) cleaves the proprotein on the way through the cell (Orci et al., 1985) to generate a mature secretory protein. In Tetrahymena thermophila this involves predominantly one form of an aspartyl cathepsin that recognises specific aspartyl residues of mucocyst Grl proteins (Kumar, Briguglio \& Turkewitz, 2014); point mutations fail to perform proper self-assembly and to acquire secretory competence.

There is reason to assume a similar mechanism for Paramecium spp. In ciliate mutants, extrusomes with non-proteolytically processed contents are not transported to the cell membrane (Pouphile etal., 1986; Tiedtke etal., 1988) - thus suggesting a probable cue for recognition by motor proteins. Previously, selection of secretory proteins by sortilin has been known from mammalian brain where it mediates the delivery of neurotrophic factor to the regulated secretory pathway (Chen et al., 2005).

Trichocysts are transported to the cell membrane according to their inherent polarity, tip first, and their secretory matrix contents provide an additional signal for docking competence (Pouphile etal., 1986). Formation of a 'tip' structure is relevant not only in Paramecium spp., but also, although structurally less impressively, in Tetrahymena thermophila. Here, unilateral assembly of one of the non-Grl proteins and of Grtlp are mandatory for exocytosis competence (Bowman et al., 2005).

When exocytotic membrane fusion is induced in Paramecium tetraurelia under conditions inhibiting decondensation/release of trichocyst contents, the trichocyst membrane reseals, the organelles become detached and undergo a new round of docking for successful exocytosis upon stimulation under standard conditions (Klauke \& Plattner, 2000). This phenomenon of 'frustrated exocytosis' indicates the occurrence of additional signals provided by the filling state that mediate competence for docking of trichocysts at the cell membrane. Such signals can include physical links between the contents of a trichocyst and its membrane, to be visualised only with appropriate fast-freezing methods (Momayezi et al., 1993).

Granule proteins similar to some of the Tetrahymena spp. mucocyst proteins are the trichocyst matrix proteins (tmp) of P. tetraurelia (Garreau de Loubresse, 1993) and Pseudomicrothorax dubius (Peck, Swiderski \& Tourmel, 1993). From analyses with Tetrahymena thermophila one may conclude that the tip part of extrusomes is probably delivered by vesicles separate from those producing the 'body/matrix' 
(Briguglio etal., 2013). The degree of acidity of the lumen of mucocyst precursors remains to be established, but probably it may not differ from the situation described for Paramecium spp.

Considering widely different secretory contents, several cues may govern extrusome biogenesis in the two genera, Paramecium and Tetrahymena, respectively. Clathrin-mediated budding of some vesicles also requires an adaptor protein complex. For instance, AP3 contributes to mucocyst biogenesis (Briguglio et al., 2013). In T. thermophila, a small GTPase, type TtRabD41 is recognised on docked mucocysts (Bright et al., 2010).

Early endosomes (called 'terminal cisternae' in Paramecium) and late endosomes ['acidosomes' in Paramecium (Allen, Ma \& Fok, 1993)], phagosomes of different age after pinching off, and diverse recycling vesicles are endowed with a V-type $\mathrm{H}^{+}$-ATPase (Wassmer et al., 2005, 2006), with v- and t-type SNAREs (Plattner, 2010b) and with CRGs (Ladenburger \& Plattner, 2011). They all vary from organelle to organelle. Participation of the plasmalemmal t-SNARE, PtSyx 1, in trichocyst exocytosis has been derived from immuno-localisation and gene-silencing experiments (Kissmehl etal., 2007). In Tetrahymena thermophila and Paramecium tetraurelia, organelles along the endocytotic pathway down to phagosomes and lysosomes dispose of organelle-specific small GTPases (Bright et al., 2010). Thus, signalling follows the established pathway in these organelles, although trichocysts exhibit several features that are unusual for dense core-secretory organelles, i.e. neutral $\mathrm{pH}$ and absence of any detectable $\mathrm{Ca}^{2+}$, as outlined above.

A tentative identification of a Rabll effector protein has been presented for T. thermophila (Zweifel et al., 2009). The intracellular distribution and dynamics of CDA12 and CDA13 (CDA = cell division protein A) during conjugation and cell division is comparable to family of Rab1 1-interacting proteins (FIPs) in mammalian cells (Fielding et al., 2005). In particular, Rabll would be interesting because it is known from mammalian cells to be a master switch for the regulation of cell surface biogenesis, structure and function (Welz, Wellbourne-Wood \& Kerkhoff, 2014); this is achieved by regulating widely diversified trafficking pathways.

\section{(3) Support by F-actin, SNAREs and $\mathrm{Ca}^{2+}$-dependent proteins}

In higher eukaryotes, actin is another regulator of membrane fusion and fission (scission), from exocytosis (Geron, Schejter \& Shilo, 2013) and endocytosis (Mooren, Galletta \& Cooper, 2012) to phagosome formation and processing (see Section V). With ciliates, such analyses have long been hampered by the insensitivity of some of the actin isoforms to diagnostic fluorescently labelled drugs (Sehring et al., 2007a). Thus, the expected involvement of $\mathrm{F}$-actin in endocytosis via parasomal sacs could not be documented. Only by gene silencing studies could PtAct4 be identified as a key player (Sehring, Reiner \& Plattner, 2010) and this isoform, in fact, does not possess normal phalloidin binding signatures (Sehring et al., 2007a).
Finally, $\mathrm{Ca}^{2+}$ has to be provided also in ciliates for promoting organelle interactions up to fusion. As specified below, this is achieved by PtCRCs specifically localised to, or indirectly associated with the different trafficking compartments (Ladenburger \& Plattner, 2011). Briefly, for trichocyst exocytosis $\mathrm{Ca}^{2+}$ is provided by release from the cortical stores, the alveolar sacs, and by superimposed influx from the outside medium (Plattner \& Klauke, 2001). For further details, see Section VIII. Local $\mathrm{Ca}^{2+}$ signals appear indispensable also for other trafficking organelles which also contain PtCRCs, although functional scrutiny of such $\mathrm{Ca}^{2+}$ signals is still missing for most organelles in ciliates.

For none of the trafficking vesicles of ciliates has any $\mathrm{Ca}^{2+}$-sensor protein been ascertained which, in metazoans, is mainly synaptotagmin. This is a single-pass membrane protein with an amino-terminal anchor and two $\mathrm{Ca}^{2+}$-binding loops attached to $\beta$-barrel motifs called C2A and C2B domains (Rizo etal., 2006). A double C2-domain protein, DOC2 (double C2-like domain containing protein), enables microneme exocytosis for host cell invasion by Apicomplexa, although it lacks a transmembrane domain (Farrell et al., 2012) - in contrast to synaptotagmin. Remarkably the existence of this molecule type has been suggested for $P$. tetraurelia although further scrutiny is required. In the Paramecium database only related proteins with eight $\mathrm{C} 2$ domains can be found ( $\mathrm{R}$. Kissmehl \& H. Plattner, unpublished observation). Similar CaBPs with more than two C2 motifs are also established for some metazoan cells where they can substitute for synaptotagmin (Min, Chang \& Südhof, 2007; Martens \& McMahon, 2008). In addition, in mammalian cells, such 'extended synaptotagmins' are also able to connect cortical $\mathrm{Ca}^{2+}$ stores [endoplasmic reticulum (ER)] with the plasma membrane (Giordano et al., 2013) - an interesting aspect, considering the likely absence of classical connections by Orai/Stim proteins in ciliates. Despite the potential importance of such C2-domain-carrying proteins for signalling none of these functions has been analysed in ciliates as yet. Docking of trichocysts and assembly of functional exocytosis sites in Paramecium tetraurelia requires calmodulin (Kerboeuf et al., 1993) and CaM is enriched in such sites (Plattner \& Klauke, 2001).

In multicellular organisms, SNAREs occurring on delivery vesicles (v-SNAREs) usually contain an $\operatorname{Arg}(\mathrm{R})$ residue in the central layer of their SNARE domain, whereas target(t-)SNAREs usually contain a Glu (Q) residue (Fasshauer etal., 1998; Sutton etal., 1998). However, like elsewhere, there are also exceptions to this rule in Paramecium tetraurelia (Plattner, 2010a,b). The SNARE domain is essential for pairing of SNAREs in trans-position which allows for subsequent membrane fusion (Jahn \& Scheller, 2006; Jahn $\&$ Fasshauer, 2012). Whereas the central amino acid is not essential and because of the absence of any stringent cues for the assignment of $\mathrm{v}$-/R- and t-/Q-SNAREs, respectively, to certain organelles, association with organelle-specific small GTPases is essential (Novick \& Zerial, 1997; Zerial \& McBride, 2001; Grosshans et al., 2006). 
In ciliates, syntaxin homologs generally can be attributed more easily to specific membranes, comparable to the situation in different systems; this is not so easy with synaptobrevins. As mentioned above, in Paramecium tetraurelia many of the 'synaptobrevins' contain an additional longin domain. Examples are PtSyb1, PtSyb2, PtSyb3, PtSyb6, PtSyb7, PtSyb8, and PtSyb9 (Schilde etal., 2006, 2010). Longins are abundant also in plants where they contribute to organelle targeting (Rossi etal., 2004), but such effects have not been analysed in ciliates as yet. Altogether cues for specific organelle targeting of/by these SNAREs, particularly of 'synaptobrevins', are not so obvious, particularly in ciliates. As found by the analysis of exocytotic mutants of Paramecium tetraurelia, in the secretory cycle additional proteins play different roles that still have to be specified (Vayssié etal., 2000; Froissard et al., 2004).

Clear vesicles, likely candidates for the biogenesis of the cell membrane and of alveolar sacs, can be recognised only under specific conditions. For instance, vesicle docking is 'frozen' when docking is allowed under conditions where fusion with their target membrane is inhibited by silencing the SNARE-specific chaperone, N-ethylmaleimide-sensitive factor (NSF) (Kissmehl etal., 2002; Schilde etal., 2010). This suggests the involvement of SNAREs also in organelle biogenesis and in cell membrane formation by constitutive exocytosis in ciliates. Constitutively active vesicles that contribute to cell membrane formation during cytokinesis can be observed in non-manipulated cells. They contain PtCRC-V-4 (Ladenburger \& Plattner, 2011), whereas in ciliates SNAREs have not yet been identified directly in the cleavage furrow. For good reasons the designation of constitutive exocytosis as 'non-regulated exocytosis' in the past has been abandoned more recently. Moreover, NSF silencing induces an impressive amount of autophagic vacuoles, wherein NSF is degraded, while cells continuously decrease in size (Kissmehl etal., 2002). Very recently, autophagic vacuole formation is recognised also to involve specific SNAREs in higher eukaryotes (Shen \& Mizushima, 2014).

Empty membranes ('ghosts') formed in the course of trichocyst exocytosis-coupled endocytosis are internalised, and this process is also accelerated by increased $\left[\mathrm{Ca}^{2+}\right]_{\mathrm{i}}$ generated during exocytosis stimulation (Plattner et al., 1997). Under our standard conditions exo-endocytosis coupling is accomplished within $0.35 \mathrm{~s}$ (Knoll, Braun \& Plattner, 1991a). Comparably rapid coupling has recently been documented, also using quenched-flow/cryofixation methodology, in nerve terminals, also via smooth vesicles (Watanabe etal., 2013). In Paramecium tetraurelia, this process is different from endocytosis via clathrin-coated pits represented by parasomal sacs. Here, endocytosis may be ascribed to the activation of calcineurin, a $\mathrm{Ca}^{2+} / \mathrm{CaM}$-activated protein phosphatase (PP2B) that is enriched on Paramecium's parasomal sacs (Momayezi etal., 1996) - the sites of endocytosis by clathrin-coated vesicles. This function is suggested by the scission of clathrin-coated vesicles by dynamin [identified in Tetrahymena thermophila (Elde etal., 2005)] which, as shown in mammalian cells, has to be dephosphorylated by calcineurin to become active (Marks \& McMahon, 1998; Lai et al., 1999).

\section{THE PHAGOGYTOTIC GYGLE}

Phagocytosis of particles and bacteria was detected around 1900 by Metchnikoff in phagocytes from starfish larvae. Soon he extended this discovery to mammalian defence mechanisms against pathogenic microorganisms. In mammals, cues regulating phagocytosis involve actin filaments, acidification (by $\mathrm{H}^{+}$-ATPase acquired from endosomes) of phagocytotic vesicles (phagosomes) and fusion with lysosomes. The phagolysosomes thus formed are able to digest foreign materials (Underhill \& Goodridge, 2012) and ciliates can, thus, feed on bacteria.

This section outlines the following aspects in ciliates (Fig. 5). As in other systems, there are multiple inputs into the phagosomal system of ciliates (Fok \& Allen, 1993). At the cytopharynx, from where phagosomes (food vacuoles) are formed, widely different signals converge. This includes microtubular arrays, called '(post)oral fibres' in Paramecium and 'deep fibres' in Tetrahymena, emanating from here to the cytoproct (the site of spent vacuole exocytosis) and to deeper cytoplasmic regions; they serve to guide recycling vesicles and other vesicle input (Allen, 1974). Late endosomes ('acidosomes'), vesicles recycling from progressive phagolysosomal stages, and 'discoidal' vesicles recycling from the cytoproct after release of undigested material all contribute to food-vacuole formation, in addition to fusion with lysosomes for further maturation (Allen \& Fok, 2000). Acidification by the $\mathrm{V}$-type $\mathrm{H}^{+}$-ATPase, provided by fusion of acidosomes/late endosomes, is important, as are changing sets of GTPases, SNAREs, actin isoforms and CRCs. Altogether they regulate multiple specific fusion/fission processes.

\section{(1) Role of $\mathrm{H}^{+}$-ATPase, GTPases and SNARE proteins}

Many signals are required to guide a food vacuole through a ciliate cell (cyclosis) and basic mechanisms may be quite similar in different species. Using monoclonal antibodies (when the molecular biology of ciliates still was in its infancy) the phagosome cycle was scrutinised in Paramecium multimicronucleatum (Fok \& Allen, 1993; Allen \& Fok, 2000). Among recycling vesicles, $100 \mathrm{~nm}$-vesicles were retrieved from advanced stages of food vacuoles and 'discoidal vesicles' from the cytoproct, all travelling along cytopharyngeal microtubular ribbons in minus-end direction using dynein as a motor (Ishida et al., 2011). Thus, there are clear-cut long-range signals for specific membrane delivery, supplemented by the following short-range signals. Actin assembly on a nascent phagosome is a signal for pinching off. This is followed by fusion with acidosomes/late endosomes (Allen et al., 1993) endowed with a $\mathrm{H}^{+}$-ATPase (Wassmer 


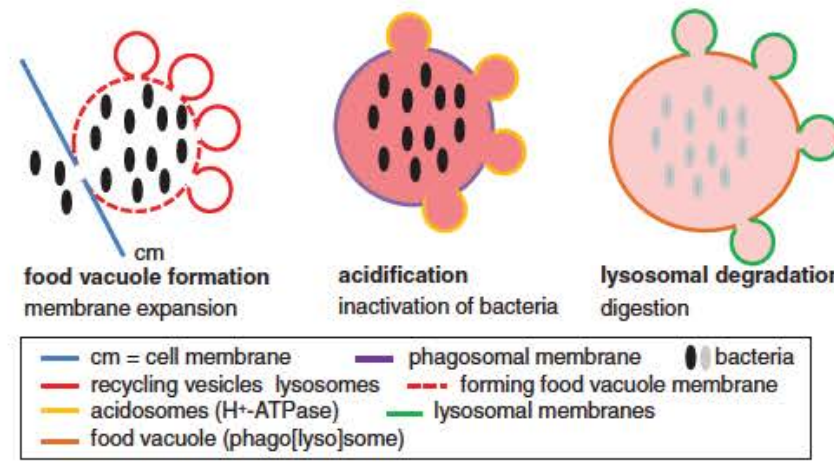

Fig. 5. Formation of a food vacuole in a ciliate requires several signalling steps. First, fusion of recycling vesicles causes the cell membrane at the cytopharynx to bulge in, trapping bacteria. Second, fusion of acidosomes (late endosomes) allow for acidification of the phagosome lumen and inactivation of bacteria. Third, fusion with lysosomes enables degradation of bacteria by acid hydrolases in the food vacuole which, thus, has become a phagolysosome. For discussion of involvement of SNAREs, CRCs, etc., see text.

et al., 2005, 2006, 2009). Silencing of some of the $\mathrm{H}^{+}$-ATPase subunits inhibits phagosome formation (Wassmer et al., 2005). Acidification is necessary not only for activating lysosomal enzymes after phagosome-lysosome fusion, but is also a signal for travel onwards through the cell. The lumenal $\mathrm{pH}$ increases steadily to a neutral $\mathrm{pH}$ before vesicles reach the cytoproct.

To understand phagosome cyclosis in ciliates, comparison with higher eukaryotic cells may be useful. The latter are paradigmatic for the sequential assembly and role of $\mathrm{H}^{+}$-ATPase, actin, small GTPases and SNAREs. For instance, in yeast, vacuoles (acidic lysosome-related compartments), specific SNAREs and Rab proteins are required for the assembly of actin (Karunakaran et al., 2012). In kidney cells, actin contributes to the targeting of some subunits of the $\mathrm{H}^{+}$-ATPase to organellar membranes (Beyenbach \& Wieczorek, 2006). Paramecium tetraurelia has the highest number of genes encoding the a-subunit of the integral V0-part of the $\mathrm{H}^{+}$-ATPase, many more than in yeast or mammals (17 versus 1 and 4 genes, respectively). Since the V0 part associates reversibly with the catalytic $\mathrm{Vl}$ head part via the a-subunit one can expect site- and time-variable association of different $\mathrm{Vl}$ parts depending on the actual requirements - an aspect suggesting extreme fine-tuning in ciliates. Also unexpected is organelle targeting by the carboxy-terminal half of the a-subunit molecule in Paramecium tetraurelia (Wassmer et al., 2006), rather than by the amino-terminal half as in yeast (Kawasaki-Nishi et al., 2001). In kidney proximal tubule cells, acidification of the organellar lumen can cause binding of small GTPases and their modulators (Hurtado-Lorenzo et al., 2006; Brown et al., 2009), which can direct vesicles, from the Golgi apparatus on, in cooperation with the t-SNARE Syx 1 (Schwartz et al., 2007). The question arises whether a similar targeting mechanism may be applicable to phagosomes in ciliates.
In Paramecium tetraurelia, Rab7, typical of lytic organelles, is bound to vesicles in the oral apparatus and on phagolysosomal membranes (Surmacz, Wiejak \& Wyroba, 2006; Osinska et al., 201 1); post-transcriptional gene silencing inhibits food vacuole formation. Specific SNAREs type $P t$ Syx and $P t$ Syb are also exchanged during phagosome cyclosis (Kissmehl et al., 2007; Plattner, 2010a,b), as are $\mathrm{H}^{+}$-ATPase subunits (Wassmer et al., 2005, 2006, 2009). Therefore, one may well expect, in ciliates, interaction of specific isoforms of these proteins along the phagocytotic pathway and interdependent signalling for targeting to specific vesicles along this pathway.

Proteome analysis of phagosomes from phagotrophic cells, including many protozoa that obtain energy by digesting phagocytosed microorganisms, revealed several important details (Goyette etal., 2012): (i) membrane composition changes during maturation, (ii) $\mathrm{H}^{+}$-ATPase, small GTPases as well as proteins relevant for transmembrane transport processes and trafficking abound, (iii) other types of short-range signalling molecules, although less abundant, also contribute. For instance, in macrophages, Syx 7 is an important phagosome SNARE. In Paramecium tetraurelia, $P t$ Syx 7 is also one of the phagosome-resident SNAREs, integrated into the phagosome membrane at a later stage of cyclosis (Kissmehl et al., 2007).

Mass spectroscopy of purified phagosomes revealed 453 peptides of which 73 were putative phagosomal proteins and 28 known for their engagement in phagocytosis in other organisms (Jacobs et al., 2006). Among membrane proteins relevant for signalling, $\mathrm{Ca}^{2+}$-ATPase, $\mathrm{H}^{+}$-ATPase subunits, Rab proteins type 1,7 , and 13 as well as actin-binding proteins were identified, together with fragments of a synaptobrevin-like protein. Functional knock-out studies with Tetrahymena thermophila suggest vacuolar protein sorting proteins, $7 t \mathrm{VPS} 13 \mathrm{~A}$ and $T t$ VPS13C, as accelerators of multiple vesicle fusions with the phago(lyso)some (Samaranayake, Cowan \& Klobutcher, 2011). In macrophages, Rab20 is an immune-regulated phagosomal membrane constitutent (Pei etal., 2014), whereas in $T$. thermophile $T t$ Rab20 is associated with cytoproct-localised phagosomes (Bright et al., 2010).

\section{(2) Role of actin, $\mathrm{Ca}^{2+}$ and $\mathrm{Ca}^{2+}$-binding proteins}

The occurrence of actin throughout a Paramecium cell, with isoforms on specific trafficking organelles (Sehring etal., 2007b), likely reflects important functions. This is also suggested by the situation in higher eukaryotes where $\mathrm{F}$-actin is co-assembled with a selection of proteins at sites of clathrin-mediated endocytosis, sorting endosomes, at the trans-Golgi network, and along the secretory pathway, etc. (Anitei \& Hoflack, 2012). Such trafficking pathways can contribute to phagosome formation where requirement of actin is particularly well established (Flannagan, Jaumouille \& Grinstein, 2012).

Actin assembly is unequivocally considered crucial for formation of a phagosome, also in ciliates. In Tetrahymena thermophila the actin-binding protein 'actin depolymerising 
factor' (ADF)/cofilin binds to the nascent food vacuole where it promotes actin polymerisation (Shiozaki etal., 2013). In Paramecium tetraurelia actin isoforms and their arrangement on phagosomes change during the phagosome cycle (Sehring et al., 2007b). This can make docking sites available, in a time-variable sequence, for different organelles, such as endosomes and lysosomes. It may also provide cues for fusion and fission processes and perhaps it is also relevant for motor functions to drive cyclosis. In this regard it is interesting to examine how and where actin can interact with some of the many myosin forms found in ciliates (Eisen etal., 2006; Coluccio, 2008). Interaction with some of the cortical myosin species (Garcés, Hoey \& Gavin, 1995) has long been considered important for cyclosis. Accordingly, travelling of food vacuoles in Tetrahymena thermophila has been shown to depend on actin and myosin type Myolp (Hosein, Williams \& Gavin, 2005).

A role for actin-binding proteins (Watanabe et al., 1998) has been documented in Tetrahymena pyriformis. Disruption of F-actin by cytochalasin D or latrunculin A (Zackroff \& Hufnagel, 2002) or stabilisation of F-actin by injected phalloidin (Kersken etal., 1986) inhibits food vacuole formation. Silencing of specific actin isoforms disturbs cell morphology and phagosome formation (Sehring etal., $2007 a, b)$. However, any specific role of changing actin isoforms in Paramecium tetraurelia (Sehring et al., 2007b) in the regulation of vesicle docking and fusion/fission processes along this route remains to be established. In fact, in macrophages, actin can either activate or inhibit fusion of phagosomes with lysosomes (Liebl \& Griffiths, 2009). Therefore, the role of changing actin isoforms in Paramecium spp. may also reflect multiple functions.

Immuno-localisation studies suggest that constitutive $\mathrm{Ca}^{2+}$ signalling plays some role during phagosome formation. CaM and CaM-BPs are bound to an early phagosome stage in Tetrahymena thermophila (Gonda, Komatsu \& Numata, 2000). CaM is associated with food vacuoles also in Paramecium tetraurelia (Maihle etal., 1981; Momayezi etal., 1986). The significance of CaM, although well documented for nascent and older stages of food vacuoles in Paramecium tetraurelia (Momayezi et al., 1986) and for large (food) vacuoles in Tetrahymena thermophila (Maihle etal., 1981), remains to be analysed. Such analyses should include CaM-BPs and it reflects the inhibition of food vacuole formation by the reliable CaM inhibitor, W7 (Gonda et al., 2000). The multiplicity of fusion processes are concordant with variable isoforms and CRCs throughout the phagocytic cycle in Paramecium spp. (Ladenburger \& Plattner, 2011). For further details about $\mathrm{Ca}^{2+}$ signalling in the endo-phago-lysosomal cycle, see Section VIII.

\section{(3) Proteins at the cytoproct}

The cytoproct also requires specific signals, or molecular cues, for its function. Here, specific Rab proteins are found in Tetrahymena thermophila (Bright etal., 2010): TtRabD19, TtRabD20, TtRabD30 and TtRab4A, all in the cytoproct region. In Paramecium tetraurelia, the same holds for proteins crossreacting with antibodies against mammalian annexins (Knochel et al., 1996), SNAREs of type PtSyb6 (Schilde et al., 2006) and PtSyx 1 and PtSyx 4 on membranes surrounding the cytoproct region (Kissmehl et al., 2007), as well as actin isoform PtActl (Sehring et al., 2007b).

\section{SIGNALLING IN THE GONTRAGTILE VACUOLE COMPLEX}

Osmotic and ionic imbalance in freshwater ciliates, resulting from ongoing permeation, requires permanent counteraction by the contractile vacuole complex (Allen \& Naitoh, 2002; Allen, Tominaga \& Naitoh, 2009). Therefore, many freshwater protozoa and algae contain a contractile vacuole complex (Hausmann, 1978). Most molecular data pertinent to organelle function come from Paramecium tetraurelia and Tetrahymena thermophila, as summarised recently (Plattner, 2013a). The molecular inventory of this complex organelle, briefly outlined in Fig. 6, includes a V-type $\mathrm{H}^{+}$-ATPase, CRCs, SNAREs, and many other components.

\section{(1) Signals for contractile vacuole biogenesis}

Taking into account the numerous additional molecules known from other protozoa, a new hypothetical picture emerges for interaction of components during the biogenesis and rhythmic function of this organelle (Plattner, 2013b). Each of these two aspects is based on multiple signalling events.

Particularly intriguing is the primary signal for formation of a nucleation site enabling de novo biogenesis of a new contractile vacuole complex. Strikingly this generally occurs just before cytokinesis and involves components that are relevant also for cytokinesis. As summarised by Plattner (2013b), it starts with the new pore (site of vacuole fusion with the cell membrane for contents release by exocytosis). Here centrin, CaM, $\gamma$-tubulin, and NIMA kinase (all known for their relevance to cytokinesis; NIM = never in mitosis) are enriched and, thus, may belong to the cues for nucleation of a pore precursor. The NIMA-related protein kinase family is greatly expanded in T. thermophila (Eisen et al., 2006). Growth of microtubules, from the pore to the very tip of radial (collecting) canals may be restricted by 'microtubule destabilizing kinesins' related to kinesin family (kif) proteins. These are kinesin-like proteins that are mainly known from metazoans (Walczak, Gayek \& Ohi, 2013), but that are also abundant in Tetrahymena spp. (Wickstead \& Gull, 2006).

Growing microtubules are accompanied by canal-forming membranes from where a widely branched spongiome emerges (Allen \& Naitoh, 2002). The distal part of the spongiome is studded with catalytic V1 parts of the V-type $\mathrm{H}^{+}$-ATPase (Fok et al., 2002). All other parts of the proximal/smooth spongiome, contain $\mathrm{Ins}_{3} \mathrm{R}$-type $\mathrm{CRC}$, type PtCRC-II-2 and PtCRC-II-3, and the RyR-related PtCRC-V-4 as well as several t-/Q- and v-/R-SNAREs. 


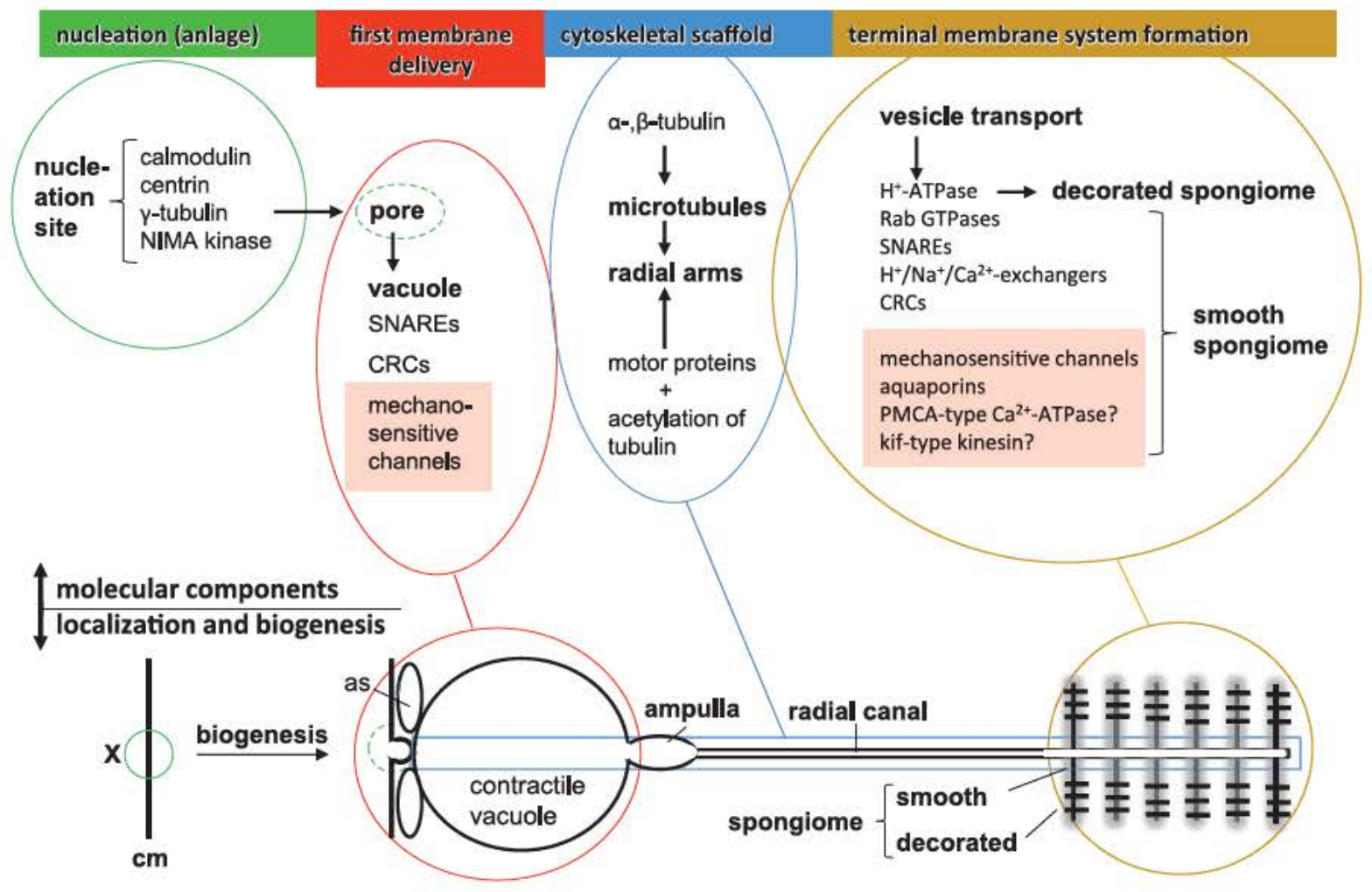

SNARES at the pore: PtSyb2, PtSyb9, PtSyx2, PtSyx15 molecules postulated CRCs at the pore (no Ins $\mathrm{P}_{3}$-binding domain): PtCRC-VI-2, PtCRC-VI-3

Fig. 6. The microscopic structure of the contractile vacuole complex (lower part of figure) in relation to the molecular constituents of the respective domains, from the pore (site of periodic contents release), via the contractile vacuole, an enlarged domain (ampulla) connecting the vacuole to radial arms, and finally to the end region, the spongiome. Data combined from different species according to Plattner $(2013 a, b)$. Six or more arms give the organelle a star-like appearance. The spongiome is endowed with $\mathrm{H}^{+}$-ATPase molecules in its outermost part (decorated spongiome) that is continuous with the smooth spongiome. This domain harbours SNARE proteins and $\mathrm{Ca}^{2+}$-release channels (CRCs), type PtCRC-II $\left(\mathrm{IP}_{3} \mathrm{Rs}\right)$, serving for the constitutive partial reflux of $\mathrm{Ca}^{2+}$ into the cytosol. The cell membrane/vacuole connection as well as the vacuole/ampulla connection are periodically dis- and reconnected in $\sim 10 \mathrm{~s}$ intervals, during cycles of systole and diastole, and, thus, require signalling. Presumably this is also the case during restructuring of the spongiome in the course of activity cycles. Accordingly, the presence of CRCs, SNAREs, GTPases, and possibly also of mechanosensitive channels, strongly suggest extensive signalling processes in this organelle. The vacuole filling state may be sensed by (still hypothetical) mechanosensitive channels, directly or indirectly causing $\mathrm{Ca}^{2+}$ signal formation and membrane fusion via SNAREs. Biogenesis of the organelle is under epigenetic control and starts with nucleation signals and formation of an anlage at the prospective pore, followed by expansion along microtubules with post-translationally modified tubulins and restriction of radial arm/canal length by a microtubule-destabilizing motor protein related to kif. For further molecular details, see Plattner (2013a,b). The release of water and $\mathrm{Ca}^{2+}$ by the vacuole is enabled chemiosmotically by $\mathrm{H}^{+}$sequestered via $\mathrm{H}^{+}$-ATPase activity. Although a plasma membrane $\mathrm{Ca}^{2+}$-ATPase (PMCA)-type $\mathrm{Ca}^{2+}$-ATPase/pump may occur in the organelle, experiments strongly suggest a preponderant role of $\mathrm{H}^{+} / \mathrm{X}^{+} / \mathrm{Ca}^{2+}$ exchangers (see text). Abbreviations: as, alveolar sac; cm, cell membrane; X, nucleation site. See Appendix (Section XIX) for other abbreviations.

Among them, PtSyb2 and PtSyx2 occur also at the pore, together with PtSyb9 and PtSyx 15 (Plattner, 2013a,b).

\section{(2) Signalling in the mature contractile vacuole complex}

The presence of $\mathrm{H}^{+}$-ATPase, SNAREs and CRCs (Plattner, $2013 a, b)$, together with the existence of specific Rab-type GTPases, identified in Tetrahymena spp. and Paramecium spp. (Bright et al., 2010), stongly suggests several roles for SNAREs.
This encompasses membrane dynamics, including $(i)$ ongoing vesicle delivery, (ii) reversible fusion/fission processes within the smooth spongiome and (iii) exocytotic fluid expulsion at the pore. (iv) $\mathrm{H}^{+}$sequestration drives sequestration of water and $\mathrm{Ca}^{2+}$ in exchange for $\mathrm{H}^{+}$, and $(v)$ some of the $\mathrm{Ca}^{2+}$ is released back into the cytosol for fine-tuning and for mediating ongoing membrane fusion processes within the contractile vacuole complex.

Which $\mathrm{Ca}^{2+}$ signals are to be expected and why? Irregular, stochastic $\mathrm{Ca}^{2+}$ puffs are registered with fluorochromes 
along radial arms; they are attributed to constitutive activity of $P t$ CRC-II/Ins $P_{3} \mathrm{R}$ channels (Ladenburger et al., 2006). The presence of stomatin, which in Paramecium tetraurelia is found from the peripheral parts of the radial arms up to the pore (Reuter, Stuermer \& Plattner, 2013), suggests the occurrence of mechanosensitive channels since both molecules are always combined with each other in other systems (see Section VIII.2). Therefore, increasing pressure may activate such channels, possibly in conjunction with some of the other CRCs. The contractile vacuole pore region in Paramecium tetaurelia contains RyR-type CRGs, type PtCRG-VI-2 and PtCRC-VI-3 (Ladenburger \& Plattner, 2011). Not only periodic vacuole emptying may thus be regulated, but also periodic de-/recoupling of the ampullae - the swollen end pieces of radial canal - a phenomenon detected by capacitance measurements (Tominaga \& Allen, 1998; Grønlien et al., 2002). The periodic transition of flat membranes in diastole to a branched tubular network during systole can hypothetically be mediated by BAR (Bin-amphiphysin-Rvs)-type proteins that are known from mammalian cells to serve this transformation (Mim \& Unger, 2012; Shen, Pirruccello \& De Camilli, 2012). This function for BAR proteins has been suggested also for the contractile vacuole complex of Paramecium spp. (Plattner, 2013a), based on the effect of F-BAR protein in Dictyostelium discoideum (Heath \& Insall, 2008). Similarly, Dictyostelium IBAR-null mutants have been shown to be defective in osmoregulation (Linkner et al., 2014). The signal for this transformation may reside in the decay of internal pressure in the organelle during systole.

Purinergic $\mathrm{Ca}^{2+}$ channels, e.g. type P2X, have not yet been identified at a molecular level in ciliates (Burnstock \& Verkhratsky, 2009; Coddou et al., 2011; Verkhratsky \& Burnstock, 2014). In Dictyostelium discoideum cells, in which $\mathrm{P} 2 \mathrm{X}$ is associated with Rablla and a $\mathrm{Ca}^{2+}$-regulated GAP, P2X regulates contractile vacuole activity (Parkinson etal., 2014) and becomes concentrated in those parts of the contractile vacuole membrane where the vacuole is attached to the cell membrane. Here it co-localises with Rab11. Their coassembly serves for the release of $\mathrm{Ca}^{2+}$ as a signal required for exocytotic membrane fusion. By contrast, in Tetrahymena thermophila Rab11A occurs on endosomes, whereas RabD2, D10 and D14 are seen on the contractile vacuole (Bright etal., 2010). Therefore, in ciliates, Ca ${ }^{2+}$ channels and Rab-GTPases of a type different from those in Dictyostelium discoideum may be involved in contractile vacuole discharge, though this requires more scrutiny. $\mathrm{InsP}_{3}$ does not seem to play a role in contractile vacuole fusion since the CRCs occurring in the pore region, $P t$ CRC-VI-2 and PtCRC-VI-3, are both devoid of an $\mathrm{Ins}_{3}$-binding domain (Ladenburger \& Plattner, 2011).

The identity and relevance of mechanosensitive channels as well as the occurrence of purinergic receptor-type $\mathrm{Ca}^{2+}$ channels in ciliates remain to be settled. So far the occurrence of the latter has been postulated only for the cell surface of Paramecium tetraurelia, based on the effects of exogenous GTP, i.e. periodic forward and backward swimming (see Section X.1).

\section{ADDITIONAL DETERMINANTS FOR VESICLE TRAFFICKING IN GILIATES}

Most essential molecules in addition to Rab and SNARE proteins have been identified in metazoan cells, from yeast to mammals (see Sections IV.2 and IV.3). Clathrin, APs and COPs, first identified in mammalian cells (Rothman, 2014), serve for vesicle budding from yeast to multicellular organisms. Together with specific subtypes of Rab proteins and SNAREs, subtypes of APs and COPs cooperate at specific sites of vesicle trafficking (Popoff etal., 2011; Bonifacino, 2014) and, thus, jointly help to specify delivery routes. Although COPs and APs also occur in protozoa, molecular details are limited, in some contrast to Rab and SNARE proteins. Also in ciliates numerous molecular recognition and interaction sites, in conjunction with signalling processes, collaborate to bud vesicles, then to put them in motion and finally to dock at a target membrane with which they may fuse. Regrettably, signalling in the Golgi apparatus of ciliates is still poorly understood to date.

A collection of additional proteins relevant for vesicle trafficking, from intracellular vesicle budding to endocytosis, can be identified in the Paramecium database (Plattner \& Kissmehl, 2003). This includes APs assigned in higher eukaryotes to different local events: AP1 for lysosome budding from the trans-Golgi network, AP2 for endocytosis, AP3 acting on endosomes, AP4 on trans-Golgi network, endosomes and lysosomes; furthermore, COPs for ER to Golgi and intra-Golgi transport (Malkus, Jiang \& Schekman, 2002). Clathrin is another molecular filter serving for vesicle budding from the Golgi, from endosomes and at the cell membrane during endocytosis (Arvan \& Castle, 1998; Godlee \& Kaksonen, 2013; Rothman, 2014). Specific details are known only from Tetrahymena thermophila where several APs and eight members of the dynamin family have been found, together with clathrin (Elde et al., 2005). Dynamin is a relatively large GTPase-type protein serving as a 'pinchase' for vesicle fission/scission (Hinshaw, 2000), i.e. during pinching off of vesicles. This list is complemented by GTPases of type Rab and Rho ( $\rho$ factor) and their regulators, such as GAPs (activation proteins) and GEFs (exchange factors), also found in the Paramecium database (Plattner \& Kissmehl, 2003). Many of the sequences from this database still require verification, whereas Rab-type GTPases have been more thoroughly analyed and localised in Tetrahymena thermophila (Bright et al., 2010).

As to phosphoinositides, the number of molecular species serving as potential cues for specific molecular interactions in general and as substrates for local signalling in particular is remarkably high in ciliates. A Paramecium tetraurelia and Tetrahymena thermophila genome analysis has revealed the presence of multiple phosphoinositide kinases (Leondaritis etal., 2013). Recall that in higher eukaryotes the rather specific distribution of phosphoinositides in membranes of different trafficking organelles reflects their importance for multiple signalling pathways and molecular recognition cues 
(Vicinanza etal., 2008; Michell, 2013). This aspect clearly deserves further clarification in ciliates.

A considerable problem in ciliates is the identification of the Golgi apparatus. Our tentative assignment of PtSyx5 to the Golgi (Kissmehl etal., 2007) is supported by the assignment of this SNARE to this organelle in higher eukaryotes (Malsam \& Söllner, 2011; Hong \& Lev, 2014). Moreover, in ciliates, it would be rewarding to analyse e.g. sec22 (SNARE binding to COPII) for identifying the Golgi apparatus. Unfortunately a homologue was found only for Tetrahymena thermophila (which was not further persued), but not for Paramecium tetraurelia (Schilde et al., 2006). Attempts to identify the Golgi apparatus by reliable markers established in mammalian cells and indicated in the Paramecium database have failed (Schilde etal., 2006). Therefore, currently only PtSyx5 remains as a tentative marker (Kissmehl et al., 2007). In Tetrahymena thermophila, Tt Rab6 subtypes A to D have been assigned to the Golgi apparatus, probably for retrograde transport (Bright et al., 2010), and related sequences can be retrieved from the Paramecium database.

\section{WHY THIS MULTIPLICITY OF $\mathrm{CA}^{2+}$-RELEASE GHANNELS (GRCs) IN GILIATES?}

In all eukaryotic cells $\mathrm{Ca}^{2+}$ is widely used as a messenger for many processes. This was first recognised for stimulus-contraction coupling in mammalian cells and, from the 1970s on, for stimulus-secretion coupling (Rubin, $1974,2012) \cdot \mathrm{Ca}^{2+}$ regulates many processes even deep inside cells (Berridge et al., 1998, 2003; Clapham, 2007). Therefore, selectively to address deep targets, $\mathrm{Ca}^{2+}$ has to be provided at defined sites in microdomains for short periods (Berridge, 2006). This can be achieved anywhere in the cytoplasm by $\mathrm{Ca}^{2+}$ release via CRCs from stores scattered throughout the cytoplasm. Therefore, different trafficking vesicles are also endowed with CRCs. In ciliates, the only known and most important types of CRCs are $\mathrm{IP}_{3} \mathrm{Rs}_{\mathrm{s}}$ and RyRs, both detected and specified primarily in mammalian cells (Bezprozvanny, 2005; Lanner et al., 2010; Hamada \& Mikoshiba, 2012). Only recently have CRCs been identified in ciliates.

This section addresses the molecules required for achieving local, site-specific $\mathrm{Ca}^{2+}$ signals throughout a ciliate cell (Fig. 7). In Paramecium tetraurelia cells, over 30 CRCs with characteristics of $\mathrm{IP}_{3} \mathrm{Rs}$ and/or RyRs have been identified by molecular analysis (Ladenburger \& Plattner, 2011). They are consistently distributed over organelles participating in trafficking and, thus, may provide local $\mathrm{Ca}^{2+}$ signals for membrane interactions and finally fusion, including exocytosis (Fig. 8). The multitude of CRGs in Paramecium tetraurelia, all with a characteristic distribution over specific organelles, suggests differential targeting and the capability for distinct, locally restricted signalling according to local requirements. This reflects the importance of strictly localised $\mathrm{Ca}^{2+}$ signalling, also in ciliates, as outlined in Section II.1. In the following we will discuss two CRCs analysed in Paramecium spp. in more detail.

\section{(1) InsP $3^{-}$and RyR-type GRGs}

PtCRCs encompass subfamilies I-VI, with paralogs (also called ohnologs when attributable to whole-genome duplications) designated by Arabic numbers, e.g. -1, -2 etc., within the subfamilies. Concomitantly, designations usable for molecular databases are, for instance, PtCRC-II-1 and PtCRC-II-2 for an $\mathrm{IP}_{3} \mathrm{R}$ occurring at the contractile vacuole complex, or PtCRC-IV-1 and PtCRC-IV-2 for RyR-LPs occurring in alveolar sacs (Ladenburger et al., 2006, 2009; Ladenburger \& Plattner, 2011). Let us exemplify the specific function and localisation of the best analysed PtCRCs.

Exocytosis stimulation in Paramecium spp. activates $\mathrm{Ca}^{2+}$ release from alveolar sacs (Ladenburger etal., 2009) and this is complemented by $\mathrm{Ca}^{2+}$ influx from the outside medium (Hardt \& Plattner, 2000). Titrating $\left[\mathrm{Ca}^{2+}\right]_{0}$ to values slightly below $\left[\mathrm{Ca}^{2+}\right]_{i}$ determined in unstimulated cells allows one to recognise which occurs first, $\mathrm{Ca}^{2+}$ influx or release from internal stores (Klauke \& Plattner, 1997; Hardt \& Plattner, 2000). Thus it was determined that $\mathrm{Ca}^{2+}$ signalling during stimulated exocytosis in Paramecium tetraurelia involves a mechanism called store-operated $\mathrm{Ca}^{2+}$ influx (SOC, also termed SOCE, store-operated $\mathrm{Ca}^{2+}$ entry), as in human skeletal muscle cells. $\mathrm{Ca}^{2+}$ mobilisation from the sacs is a first step that triggers influx of additional $\mathrm{Ca}^{2+}$ from the outside medium. Whereas the plasmalemmal influx channels have not been identified as yet, localisation and genetic-silencing experiments have identified RyR-LP type PtCRC-IV to mediate $\mathrm{Ca}^{2+}$ mobilisation from alveolar sacs (Ladenburger etal., 2009). RyR activators, such as caffeine and 4-chloro-meta-cresol, have been successfully applied. They all produce a $\mathrm{Ca}^{2+}$ signal for trichocyst exocytosis by the SOC(E) mechanism, whereas both phenomena are inhibited after PtCRG-IV silencing. The RyR-LP of Paramecium tetraurelia is activated not only by the orthodox RyR activators mentioned above, but also by AED (Ladenburger etal., 2009). RyR-LPs in the parts of alveolar sac membranes facing the cell membrane (Ladenburger et al., 2009) provide strictly vectorial dissipation of $\mathrm{Ca}^{2+}$ over trichocyst exocytosis sites.

$P t \mathrm{IP}_{3} \mathrm{Rs}$ bind ${ }^{3} \mathrm{H}$-inositol 1,4,5-trisphosphate as demonstrated using the isolated $\mathrm{Ins}_{3}$-binding domain after homologous overexpression (Ladenburger et al., 2006). Surprisingly the $P t \mathrm{IP}_{3} \mathrm{R}$ is localised to the contractile vacuole complex where it is constitutively active. Constitutive activity explains why other efforts to monitor $\operatorname{Ins}_{3}$ increase during stimulation have failed. Formation of $\mathrm{InsP}_{3}$ generally requires a phospholipase $\mathrm{C}$ (PLC) which, in ciliates, occurs in different isoforms (Klöppel et al., 2009; Leondaritis et al., 2011) some of which may fulfill this task. For comparison, phospholipase $\mathrm{C}$ subfamilies $\beta, \gamma, \delta, \varepsilon, \eta$ and $\zeta$ occur in animals and only type $\zeta$ in plants (Munnik \& Testerink, 2009), whereas orthologs in ciliates are addressed in Section IX.1.

$\mathrm{Ca}^{2+}$ is sequestered into the contractile vacuole complex dependent on the activity of a $\mathrm{V}$-type $\mathrm{H}^{+}$-ATPase/pump (Fok etal., 2002; Wassmer etal., 2005, 2006), probably in conjunction with a hypothetical $\mathrm{Ca}^{2+} / \mathrm{H}^{+}$exchanger (Plattner, 2013a,b). PtIP $\mathrm{IP}_{3}$ contained in the contractile 


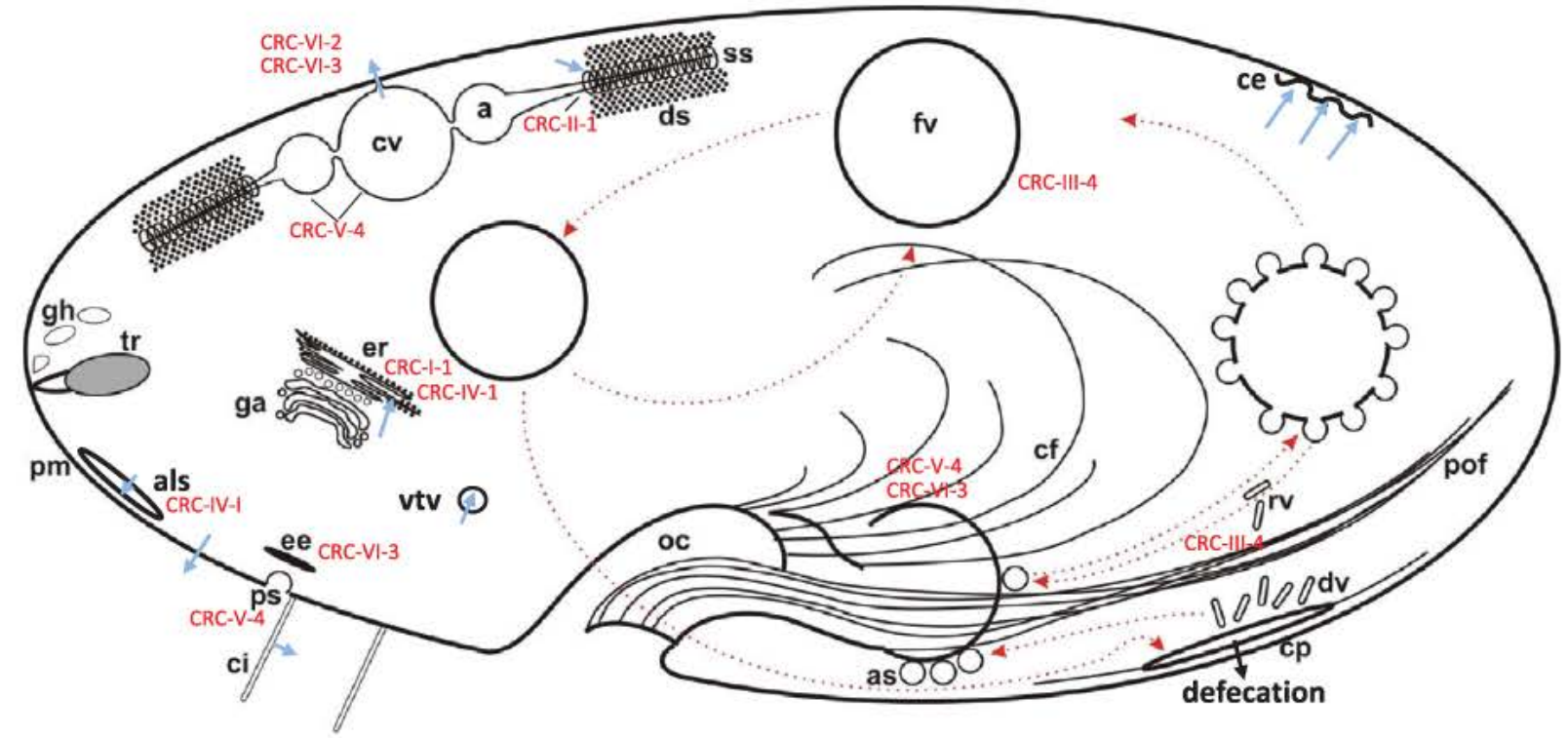

Fig. 7. Different isoforms of $\mathrm{IP}_{3} \mathrm{R} / \mathrm{RyR}$-type (PtCRCs) are distributed over different trafficking organelles of a Paramecium cell (nuclei omitted). Some organelles contain more than one type and a given type can occur in several organelles, often of the same trafficking route (red dotted arrows indicating pathways of phagocytotic cycle). Furthermore, a ciliated protozoan cell possesses several ways to eliminate $\mathrm{Ca}^{2+}$, be it during constitutive activity or after stimulation. This includes mechanisms indicated by blue solid arrows, i.e. extrusion at the cell surface, sequestration into stores and binding to $\mathrm{Ca}^{2+}$-binding proteins $(\mathrm{CaBPs})$. The latter is the most rapid means of $\mathrm{Ca}^{2+}$ downregulation after stimulation (e.g. in cilia; see Fig. 2); then, after $\mathrm{Ca}^{2+}$ desorption and dissipation, some of the slower processes can follow. Among them, extrusion via the contractile vacuole complex appears most effective, although its mode of action is not yet known in detail, secondary active $\mathrm{Ca}^{2+}$ export is most likely (Fig. 6). Refilling of alveolar sacs by a $\mathrm{Ca}^{2+}$ pump is among the slow processes, as is extrusion by the $\mathrm{Ca}^{2+}$ pump of the cell membrane. Abbreviations: a, ampulla; als, alveolar sac; as, acidosomes; ce, centrin; cf, cytopharyngeal fibres; ci, cilium; cp, cytoproct; cv, contractile vacuole; ds, decorated spongiome; dv, discoidal vesicles; ee, early endosome; er, endoplasmic reticulum; fv, food vacuole; ga, Golgi apparatus; gh, 'ghost' (empty trichocyst membrane); oc, oral cavity; pm, plasma membrane; pof, postoral fibres; ps, parasomal sac; rv, recycling vesicles; ss, smooth spongiome; tr, trichocyst; vtv, various trafficking vesicles.

vacuole complex, mainly in its smooth spongiome, allow for stochastic reflux of $\mathrm{Ca}^{2+}$ into the nearby cytosol (Ladenburger etal., 2006). As mentioned in Section VI, all this may serve for $\left[\mathrm{Ca}^{2+}\right]_{\mathrm{i}}$ fine-tuning in the cytoplasm, for ongoing vesicle delivery in the course of membrane turnover (biogenesis) and also for restructuring of the densely packed membrane tubules in the smooth spongiome (Plattner, 2013b).

\section{(2) Additional types of CRCs}

The occurrence of intracellular mechanosensitive channels in Paramecium tetraurelia cells has been postulated on the basis of the presence of stomatin in food vacuoles and in the contractile vacuole complex up to its pore region (Reuter et al., 2013). This scaffolding protein is normally associated with microdomains and, on a molecular level, with mechanosensitive channels for whose activity it is obligatory (Lapatsina etal., 2012). Build up of internal pressure during contractile vacuole diastole (Tani, Allen \& Naitoh, 2001) could, thus, be a direct or indirect signal for the activation of mechanosensitive channels and, directly or indirectly, for the release of $\mathrm{Ca}^{2+}$ (Plattner, 2013b). Any $\mathrm{Ca}^{2+}$ signal could induce the exocytosis of vacuole contents [water and ions, including $\mathrm{Ca}^{2+}$ (Allen \& Naitoh, 2002; Grønlien et al., 2002;
Stock, Grønlien \& Allen, 2002)] in a kiss-and-run mode. The periodic de- and recoupling of radial arms from/to the contractile vacuole during activity cycles, as established by electrophysiological analysis (Grønlien et al., 2002), could be regulated by a similar, pressure-induced signalling pathway.

Although this scenario of intriguing interactions still requires experimental verification, it is in line with other results. Interestingly, ceramide is a component of rafts/microdomains in mammalian cells (Simons \& Gerl, 2010) where it can regulate mechanical sensitivity in lipid bilayers (López-Montero et al., 2010). When living Paramecium tetraurelia cells are exposed to labelled ceramide, this is endocytosed and transferred to the contractile vacuole system (as well as to food vacuoles where it forms patches) (Iwamoto \& Allen, 2004), just like stomatin (Reuter etal., 2013). Microdomain-forming components could coalesce, and this perhaps includes the mechanosensitive channels in the membranes of the contractile vacuole complex. Generally mechanotransduction depends on appropriate lipid microdomains (Anishkin \& Kung, 2013).

Two groups of mechanosensitive channels, primarily conducting $\mathrm{Ca}^{2+}$ (Hamill \& Martinac, 2001), have been detected by database analysis in ciliates, i.e. Piezo (otherwise known from a mammalian neuronal cell line) 


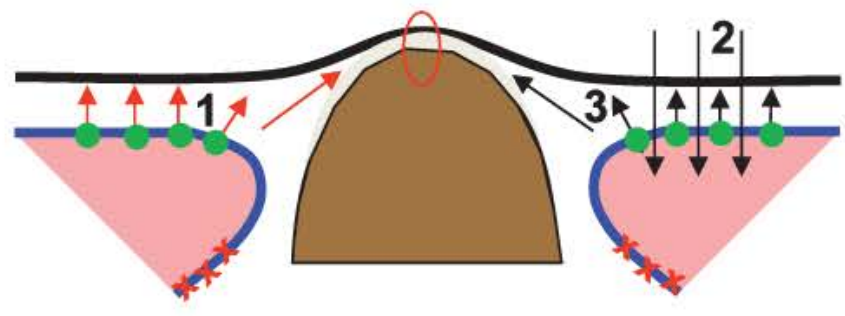

trichocyst connecting material alveolar sacs 0 preformed membrane fusion site PtCRC-IV $\uparrow \uparrow$ primary and secondary $\mathrm{Ca}^{2+}$-release $\downarrow \mathrm{Ca}^{2+}$ influx sites $(1-3$ sequence of fluxes) X SERCA-pump ( $\mathrm{Ca}^{2+}{ }^{2+}$-ATPase $)$

Fig. 8. $\mathrm{Ca}^{2+}$ signalling mediating trichocyst exocytosis in Paramecium spp. This includes a site-directed $\mathrm{Ca}^{2+}$ flow, involving steps in the following sequence (indicated by arrows, 1-3). Step 1: release of $\mathrm{Ca}^{2+}$ from cortical stores (alveolar sacs); Step 2, a $\mathrm{Ca}^{2+}$ influx; Step 3, a second wave of $\mathrm{Ca}^{2+}$ release from the sacs in a tightly coupled sequence called SOCE. The release channels involved, type PtCRC-IV, face the cell membrane, whereas the SERCA-type $\mathrm{Ca}^{2+}$ pump is localised to the opposite side. The nature of the $\mathrm{Ca}^{2+}$-influx channels involved is not yet established.

(Coste et al., 2010) as well as some transient receptor potential (TRP) channels (Patel \& Docampo, 2009; Kung, Martinac $\&$ Sukharev, 2010). In the Interpro Paramecium database, the Piezo family is represented by proteins of between 2338 and 2765 amino acids (IPRO027272), i.e. comparable to established Piezo-type mechanosensitive ion-channel components 1 and 2 of mammals. Among the large group of TRP channels some are localised to the cell membrane, some to organelles (Patel \& Docampo, 2009), including lysosomes. TRP channels contribute to phagosome formation in macrophages (Samie etal., 2013), whereas in ciliates details remain to be established.

In mammalian cells, trafficking organelles store $\mathrm{Ca}^{2+}$ to a variable extent (Hay, 2007; Sherwood et al., 2007), from early endosomes (Luzio, Gray \& Bright, 2010) to lysosomes (Christensen, Myers \& Swanson, 2002). The membranes of some of these organelles are known to contain established CRCs (Zampese \& Pizzo, 2012). Concomitantly, $\mathrm{Ca}^{2+}$ regulates phagosome formation, fusion of phagosomes with endosomes of different stages and with lysosomes (Jaconi et al., 1990; Hay, 2007; Zampese \& Pizzo, 2012). All these examples of CRCs along the endo/lyso/phagosomal pathway make us expect a comparable abundance also in Paramecium spp. (Ladenburger \& Plattner, 2011).

In Paramecium tetraurelia, effects indicative of $\mathrm{Ca}^{2+}$ signal formation have been observed after microinjection of cADPR and of NAADP (Plattner et al., 2012). These second messengers are formed from NAD and NADP, respectively, by the same cyclase, CD38 (Lee, 2012). While cADPR is discussed by some as an endogenous activator of some RyRs (Ogunbayo etal., 2011; Lee, 2012; Ernst, Fliegert \& Guse, 2013), NAADP is proposed to activate two-pore channels (TPCs) in acidic compartments (Galione etal.,
2009; Churamani etal., 2013). However, there is also evidence for co-activation of other types of CRCs (Guse, 2012) and of conductances other than $\mathrm{Ca}^{2+}$ (Morgan \& Galione, 2014). Thus, the precise mode of action and $\mathrm{Ca}^{2+}$ specificity or involvement of TPCs is not yet fully settled even in mammalian cells (Morgan \& Galione, 2014). TPCs occur in acidic compartments, e.g. in recycling vesicles, lysosomes and phagolysosomes of animals and plants, whereas only indirect evidence exists for their presence in protozoa (Patel \& Cai, 2015). In Paramecium tetraurelia, the occurrence of a protein with high-affinity NAADP binding $\left(\mathrm{K}_{\mathrm{D}}=3.3 \mathrm{nM}\right)$ has been ascertained (Plattner et al., 2012), but not investigated further. TPCs are abundant in choanoflagellates (Cai, 2008) - unikont protozoa at the base of early animal evolution; so far TPCs have not been identified at a molecular level in bikonts, such as ciliates. In summary, $\mathrm{Ca}^{2+}$ mobilisation by cADPR and NAADP and their receptors in ciliates remains to be scrutinised.

As for RyRs (or RyR-LPs) in bikonts other than ciliates, there are occasionally indirect hints for their occurrence in plants, but no molecular identification is yet available (Blatt, 2000; Munnik \& Testerink, 2009). Generally their occurrence is disputed (Edel \& Kudla, 2015). In Apicomplexa, important details of $\mathrm{Ca}^{2+}$ signalling have not been elucidated yet; for instance, molecular biology suggests the absence of $\mathrm{IP}_{3} \mathrm{R} /$ RyR-type CRCs (Prole \& Taylor, 2011; Plattner etal., 2012). This is in contrast to the persistent observation of $\mathrm{InsP}_{3}$ effects in these cells (Passos \& García, 1998; Lovett et al., 2002; Lourido \& Moreno, 2015).

\section{PROTEIN PHOSPHORYLATION INVOLVED IN SIGNALLING}

An important signalling mechanism is protein phosphorylation by kinases. In metazoans, this contributes to the regulation of metabolism, cell contraction, intracellular trafficking, secretion, transcriptional activity, apoptosis etc. Johnson, 2009). Therefore, this section outlines some basic insights into protein kinase and phosphatase activities in ciliates and also covers the controversial question of tyrosine phosphorylation. In metazoans, protein kinases include PKA, PKG (Section III.2), CaM-kinase and protein kinase C (PKC) (see below) etc. Whereas in animal cells phosphorylation of seryl and threonyl residues prevail in cytoplasmic signalling, activation of some cell-surface receptors and some phenomena connected to cell cycle activity mainly involve tyrosyl phosphorylation. In ciliates, Ser and Thr phosphorylation have been confirmed, also by PKA and PKG, whereas PKC (see below) and Tyr phosphorylation by additional kinases are not well established yet.

In principle, protein modifications known from signalling mechanisms in metazoans are also found in ciliates, but they are poorly analysed in the latter. Serine/threonine phosphorylation is common, in contrast to tyrosine phosphorylation which also in ciliates is expected to be restricted to events related to the cell cycle and mitosis. Scrutiny is hampered by 
the paucity of biochemical data and subcellular localisation studies with phosphoproteins as well as of protein kinases and phosphatases, but phosphoproteome analysis currently provides an important research focus.

\section{(1) Kinases and the phosphoproteome}

Protein kinases contribute $3.8 \%$ and $\sim 7 \%$ to the genome of T. thermophila (Eisen et al., 2006; Tian et al., 2014) and P. tetraurelia (Bemm et al., 2009), respectively. Globally the kinome is larger than in mammals, not only in $P$. tetraurelia where a large fraction represents very similar ohnologs from a rather recent whole-genome duplication, but also in T. thermophila (Tian et al., 2014). We currently do not know all essential aspects of protein kinases; phosphorylation motifs predicted in $\mathcal{T}$. thermophila are shared only in part with mammals. Recent mass spectrometry-based proteome analysis in T. thermophila has identified 1384 phosphopeptides and the occurrence of $>1000$ protein kinases and protein phosphatases (Tian et al., 2014). However, since only a few phosphorylation sites have been specified in these cells, correlation with signalling processes is still difficult. Phospho-Ser/Thr forms largely prevail over the $3.8 \%$ phospho-Tyr forms. Six hundred and thirty putative kinases are considered cilia specific (Tian et al., 2014). These predictions require validation.

The field of signalling by protein phosphorylation is not very advanced in ciliates, compared with the manyfold signalling modes via Ser, Thr and Tyr phosphorylation known in mammalian cells where this takes place in cell membrane, cytosol, organelles, and the nucleus (Good et al., 2011). In ciliates, phosphorylation effects exert a broad activity spectrum ranging from metabolism and ciliary activity to surface morphogenesis. For example, phosphorylation of ciliary rootlet proteins is a signal contributing to the morphogenesis of the Paramecium tetraurelia cell cortex (Keryer et al., 1987; Sperling et al., 1991). Mps one binder (MOB1), a regulator of cytokinesis and exit from mitosis (Luca et al., 2001) that is widely distributed from protozoa to plants and mammals (Hergovich, 2011), proved essential for surface pattern formation in the ciliate, Stentor coeruleus (Slabodnick etal., 2014). The relevance of mitotic components for the de novo biogenesis and proper positioning of new contractile vacuole complexes in the cortex of Paramecium spp. has been discussed elsewhere (Plattner, 2013b).

Phosphorylation by specific Ser/Thr kinases, such as PKA, PKG, casein kinase and CDPK, as well as dephosphorylation by protein phosphatases is discussed throughout this review. For instance, for the reversible Thr and Ser phosphorylation of the exocytosis-sensitive phosphoprotein, pp63, see Section XII. Such examples are involved in signalling along different functional cascades. In the T. thermophila phosphoproteome analysis, peptide sequences characteristic not only of already known cyclic nucleotide-dependent kinase substrates have been discovered, but interestingly also for PKC types $\varepsilon$, $\delta, \zeta, \alpha / \beta / \gamma$ and $\mu$ (Tian etal., 2014). These are valuable suggestions for experimental validation, although they do not yet prove the occurrence of the respective protein kinases.
Remarkably PKC is reportedly absent from plants (Munnik \& Testerink, 2009).

Therefore, the question still is open whether ciliates possess an orthodox PInsP $P_{2}$ turnover and diacyl glycerol (DAG) formation as a PKC activator. Although this now looks very likely, experimental validation is required. Another putative Ser/Thr kinase in T. thermophila is a casein kinase which has been cloned and identified in P. tetraurelia (Vetter et al., 2003).

\section{(2) Tyrosyl phosphorylation and mitogen-activated protein kinases in ciliates?}

The effect of the phosphorylation of a Tyr residue in a protein is significantly different from the phosphorylation of other amino acid residues. This is due to the fact that the oxygen of the phenol ring sticks out from the protein backbone and, thus, makes a phospho-Tyr site accessible to other binding partners (Hunter, 2014). To date, true signalling via Tyr phosphorylation, with dedicated Tyr kinases and Tyr phosphatases, has not been reported from ciliates (Goldberg etal., 2006) or was reported as absent (Liu etal., 2011). Tyr-kinases are known from holozoans, i.e. choanoflagellates and related filastereans (Suga etal., 2014), as well as from amoebocytes (Dictyostelium spp.). In Dictyostelium discoideum, some kinases phosphorylate transcription factors, Spt6 and Stat, by dual-function kinase activity that is not specific for Tyr (Langenick etal., 2008). Such proteins contain a Src homology (SH2) domain, i.e. a phospho-Tyr binding domain [Src (name derived from sarcoma) designates a non-receptor tyrosine kinase family]. Interestingly, one such protein has been identified in the $T$. thermophila database (Manning etal., 2008; Liu etal., 2011). Here, three phospho-Tyr phosphatases have also been presumed (Manning etal., 2008). In general, however, dedicated, monospecific Tyr kinases are not considered an element of signalling in ciliates, at least not beyond mitotic/nuclear signalling. Only found from choanoflagellates onwards (Hunter, 2014), the non-Tyr-specific Thr/Tyr kinases are considered to represent the origin of metazoan genuine Tyr-kinases.

Remarkably, in mammalian cells, Src, Ras (small GTPase), mitogen-activated protein kinase (MAPK) and kinases following in a signalling cascade (see Section X) operate at many sites, from cell membrane to the transcriptional level, and so can regulate widely different functions (Avraham \& Yarden, 2011). This also includes early steps of the secretory pathway at the level of the Golgi apparatus (Farhan et al., 2010). [The latter also holds true for heterotrimeric G-proteins (Jamora etal., 1997; Michaelson etal., 2002; Coria, Masseroni \& Diaz Anel, 2014)]. However, very little is known from ciliates as yet, except for some aspects of MAPK-mediated signalling (see Section X).

The ciliate Euplotes raikovi releases a water-soluble pheromone that can exert an autocrine effect by activation of Er-MAPK (Vallesi etal., 2010). In mammalian cells, these enzymes have to be double-phosphorylated in their Thr-X-Tyr motifs to achieve full kinase activity, whereby the Tyr residue is autophosphorylated (Fu et al., 2005). It is not yet known whether this double-phosphorylation including Tyr 
autokinase activity of this kinase also occurs in Euplotes raikovi. Active MAPK typically acts as a Ser/Thr kinase which, therefore, would serve for the transmission of the signal also from the $E r$-MAPK. The receptor-ligand complex is internalised, probably not for its downregulation but rather for signal transfer from inside according to a signalosome function (Vallesi et al., 2005) familiar from mammalian cells. This aspect is followed up in Section X.

Phosphoproteome analysis in $\mathcal{T}$. thermophila strongly suggests some Ser/Thr and less Tyr-phosphorylation events along the MAPK and extracellular-signal regulated kinase (MEK), Ras and cyclin-dependent kinase ( $\mathrm{Cdk}$ ) pathways involved in signalling during cell cycle regulation and mitosis (Tian etal., 2014). Experimental verification of one MAPK type, Tt MPK3, has been achieved by expression studies in Tetrahymena thermophila where further sequences of extracellular-regulated kinase (Erk)-type proteins have also been found (Arslanyolu, 2007). Meanwhile more family members have been identified (Yildiz \& Arslanyolu, 2014). In higher eukaryotes, for instance, MEK is a Tyr/Thr kinase. Further support for such signal transduction comes from experiments with Euplotes raikovi, including heterologous analyses in mammalian cells (see Section X).

In Tetrahymena thermophila, Tyr phosphorylation of an alleged insulin receptor-like protein was derived from immunostaining of Western blots with antibodies that recognise Tyr phosphorylation sites in mammalian cells (Christensen etal., 2003). However, antibodies may also recognise a conformational motif unrelated to phospho-Tyr. Validation by biochemical analysis, e.g. matrix-assisted laser desorption/ionisation (MALDI) analysis etc., would be helpful in such cases.

In summary, no Tyr-protein kinase destined for nonMAPK-related signalling has been experimentally identified at a molecular level in ciliates as yet. This aspect currently awaits experimental scrutiny.

\section{SURFAGE RECEPTORS FOR INTRAGELLULAR SIGNALLING}

In metazoans, surface receptors are responsible for many functions, from cell survival to cell division, differentiation to apoptosis, secretion to endocytosis, chemotaxis to immune defence etc. (Gomperts et al., 2009). Most of these functions occur not only in multicellular organisms, but also in yeast and some protozoa, such as Dictyostelium spp. Which receptors occur in ciliates?

A crucial finding was signalling in neutrophilic granulocytes by a receptor for formyl-methionyl-leucylphenylalanine (fMLP) - the molecular 'trace' of bacteria which neutrophils can follow by chemotaxis for subsequent phagocytosis and inactivation by digestion. The fMLP receptor (Quehenberger et al., 1993) is an example of the large family of (trimeric) G-protein-coupled receptors (GPCRs). By combination of different ligands with different GPCR subtypes and different G-protein subunits, many different signals can be differentially transduced (Katritch, Cherezov \& Stevens, 2013). In some cases GPCR activation is followed by PKC activation. GPCRs occur in Dictyostelium discoideum and in sponges (Strotmann et al., 2011), whereas occurrence in ciliates requires further scrutiny.

When a chemical signal is released into the medium for stimulating neighbouring cells such 'paracrine' effects can be transduced via phosphorylation cascades, such as MAPKs. This applies, for instance, not only to mating-inducing pheromones released by yeast cells (Merlini, Dudin \& Martin, 2013), but also to developing heart tissue (Noireaud \& Andriantsitohaina, 2014). Paracrine signalling by released proteins is currently under scrutiny for ciliates, as discussed below. Signalling by nucleotides such as ATP, released from cells, is established in most animal kingdoms from protozoa onwards (Verkhratsky \& Burnstock, 2014). However, in ciliates important details about paracrine signalling by secreted nucleotides remain to be scrutinised.

This section highlights some of the most eminent, although only partially understood questions of signal transduction in ciliates, including the motility response to chemicals (chemokinesis) and directional orientation of motility (chemotaxis), the controversial issues of GPCRs and of MAPKs.

In Paramecium spp. and Tetrahymena spp. chemosensory transduction can involve attractants and repellents, respectively. For attractants, notably folate, acetate and glutamate, receptors are envisaged; the effects of repellents are more diffuse (Van Houten, 1998). More specifically, in Euplotes raikovi, pheromones can act via receptors not only as attractants in a paracrine mode, but also induce an autocrine signalling cascade (Vallesi et al., 2010). In Blepharisma japonica, two gamones occur: as usual, gamone 1 is a glycoprotein, whereas gamone 2 is a tryptophan derivative (Luporini, Alimenti \& Vallesi, 2014). In ciliates, evidence for trimeric G-proteins and receptor coupling is fragmentary - their occurrence is elusive. Another concern envisages purinergic receptors that have been postulated to occur in ciliates. For instance, extracellular GTP (a quite atypical activator) triggers clear-cut periodic ciliary responses, but molecular details are still missing.

\section{(1) Different types of surface receptors}

Even normal ciliate cultures require signalling in order to survive. Thus, an isolated ciliated protozoan cell requires a small volume enabling it to condition its medium by secreting proteins that act as survival factors. When transferred into too large a volume of culture medium, cells usually die. In Tetrahymena thermophila, survival factors are $\sim 10-22 \mathrm{kDa}$ in size (Rasmussen \& Wheatley, 2007). Their signalling pathway remains to be established. When Tetrahymena thermophila cells grow in synchronized cultures (not available for Paramecium) this stimulates the upregulation of over 1700 genes, among them some of the Rab protein family (Miao et al., 2009).

The bacterial chemoattractant, N-formyl-Met-Leu-Phe, known from its effect on neutrophilic granulocytes and macrophages, is reported to stimulate chemokinesis also 
in Tetrahymena thermophila (Leick, 1992). A considerable chemokinetic effect, i.e. increased swimming velocity, has been induced by a mixture of $0.2-1.5 \mathrm{kDa}$-sized oligopeptides released from Methanococcus sp. cells at concentrations of $>10^{-6} \mathrm{M}$ (Leick \& Lindemose, 2007). Any chemotactic effect promoting increased food acquirement has not been analysed.

Ciliates can secrete cell type-specific signalling substances, now called pheromones (previously called gamones or mating-type factors); they are (glyco)proteins of different types. Cell surface-bound pheromones occur in Paramecium and Tetrahymena species, whereas they are released into the ambient medium as free molecules in Blepharisma and Dileptus species, also of different species, or finally both bound and free forms occur in different Euplotes species (Luporini et al., 2005, 2014; Vallesi et al., 2008). As described in Section IX, in Euplotes raikovi a pheromone released into the medium can activate, via an autocrine pathway, a mitogenic cascade involving MAPK activity (Vallesi et al., 2010). Important signalling steps are activated when the Euplotes raikovi pheromone, Er-1, is applied to human T-cells (Cervia et al., 2013). These data suggest rather extensive effects on the promotion of mitotic activity also in Euplotes raikovi.

Depending on the Euplotes species, pheromones can also exert paracrine effects by binding to 'conspecific' cells to induce mating, i.e. sexual activity (conjugation) (Luporini etal., 2005, 2014; Vallesi etal., 2008). Similar effects are known from Blepharisma japonicum where gamone type 2 is secreted by cells of mating type II, but renders cells of mating type I ready for conjugation (Sugiura \& Harumoto, 2001). The signalling cascade thus activated must be rather complex, as this activation also includes changes in swimming behaviour and positive chemoresponses that increase the probability of encounters with conjugation partners (Sugiura et al., 2010). Generally, a non-self gamone/pheromone released by a complementary mating type slows down motility and, thus, facilitates partner pairing for conjugation (Ricci, 1996). Sumoylation, i.e. the attachment of a small ubiquitin-like modifier (SUMO), to various proteins is an important signalling process also in ciliates where it is required for cell pairing (conjugation) competence (Nasir etal., 2015) and macronuclear development (Matsuda \& Forney, 2006). Electrophysiology demonstrated a reduction of spontaneous depolarisation frequency and an increase in depolarisation- and hyperpolarisation-activated currents in a complex interplay (Stock etal., 1999). Some of these currents are $\mathrm{Ca}^{2+}$ activated, but the source of $\mathrm{Ca}^{2+}$ could not be established. Along these lines, in Paramecium bursaria, microinjection of $\operatorname{Ins}_{3}$ induces conjugation (Miwa \& Wada, 1995), yet, unfortunately, these cells also contain symbiotic Chlorella cells which could account for the effect reported. In Paramecium spp., no releasable gamones exist. Mating types are maternally inherited, i.e. by the macronucleus. Mating type $\mathrm{E}$ depends on the expression of $\mathrm{mtA}$, a membrane-spanning protein. The default type $\mathrm{O}$ is formed by the excision of the mtA promotor mediated by scnRNA activity (Singh et al., 2014).
Conjugation (see Section XV) is another process depending on surface receptors and recognition molecules. During conjugation, cell-cell fusion takes place. As in metazoans, this depends on proteins different from SNAREs. In Tetrahymena thermophila, HAP2, a transcriptional activator, was identified by green fluorescent protein (GFP)-localisation and gene deletion as a relevant protein (Cole etal., 2014) which is known to mediate gamete fusion in invertebrates. In T. thermophila, ASI2 (signal transducers and activators of transcription 2 protein) is considered a putative signal transduction receptor relevant for the transformation of the post-conjugation micronucleus to a macronucleus $(\mathrm{Li}$ etal., 2006). Knockout cells are arrested at the stage of macronuclear anlagen development and they die before post-conjugation fission.

When Paramecium tetraurelia cells are exposed to extracellular GTP, $\geq 10 \mu \mathrm{M}$, this causes periodic forward and backward swimming (different from ciliary reversal) in $\sim 8 \mathrm{~s}$ intervals; this is accompanied by oscillating membrane depolarisations (Clark, Hennessey \& Nelson, 1993) and by oscillating intracellular $\mathrm{Ca}^{2+}$ signals (Sehring \& Plattner, 2004) that are consistent with oscillating $\mathrm{Ca}^{2+}$ currents (Mimikakis, Nelson \& Preston, 1998). This effect is specific to GTP and cannot be induced by any other nucleotide triphosphate in any comparable concentration. By contrast, extracellular signalling by GTP is unusual in higher eukaryotic systems. Here, since the discovery of paracrine effects of nucleotides (Osipchuk \& Cahalan, 1992), such signalling has remained restricted essentially to ATP (Verkhratsky \& Burnstock, 2014). An extracellular receptor for ATP has recently been found also in Arabidopsis thaliana (Choi et al., 2014).

Whereas Paramecium tetraurelia is reported usually to react only to GTP (Hennessey, 2005), Tetrahymena thermophila displays repetitive back-/forward swimming not only in response to $\operatorname{GTP}(10 \mu \mathrm{M})$, but also to ATP $(\sim 500 \mu \mathrm{M}$ for maximal response) (Kim etal., 1999). The occurrence of a receptor is assumed, but the efficiency of drugs on the effects of ATP and GTP, respectively, differ greatly (Hennessey, 2005). Considering the rather problematic pharmacology of ciliates (Plattner etal., 2009) any further interpretation, e.g. on the involvement of trimeric G-proteins (see Section X.2), would greatly benefit from identification of a receptor at a molecular level. This response to nucleotide triphosphates is unexpected insofar as there are no purinergic receptors known from ciliates (Burnstock \& Verkhratsky, 2009; Coddou etal., 2011; Verkhratsky \& Burnstock, 2014), in contrast to Dictyostelium discoideum (Fountain et al., 2007). In Paramecium tetraurelia GTP-induced signalling involves release of $\mathrm{Ca}^{2+}$ from unidentified internal stores (Wassenberg, Clark \& Nelson, 1997), but a stronger first $\mathrm{Ca}^{2+}$ peak depends on $\left[\mathrm{Ca}^{2+}\right]_{\mathrm{o}}$ (Sehring \& Plattner, 2004). The biological significance of these $\mathrm{Ca}^{2+}$ oscillations and of the repetitive back/forward movement is not quite clear, but it was suggested that the GTP-induced behaviour counteracts dispersal of the cells (Sehring \& Plattner, 2004). 
Work with Paramecium and Tetrahymena species has established their capability for positive and negative chemoresponses to attractants and repellents, respectively (Van Houten, 1998). Examples of attractants are glutamate and folate. Silencing of a gene involved in the biosynthesis of the glycosyl phosphatidylinositol- (GPI-)anchor of surface proteins $(P I G-A)$, known from mammalian cells, resulted in reduced chemoattraction by glutamate and folate (Yano, Rachochy \& Van Houten, 2003). Since then, the folate receptor was assumed to be a GPI-anchored protein. In mammalian cells there is an ongoing debate about the mechanism of signal transmission by GPI-anchored proteins, considering the absence of a transmembrane domain.

Surface receptors involved in the explosive release of toxic secretory organelles, the toxicysts, are not known. In this crudest form of intercellular 'communication' toxicysts are released upon contact with other ciliates serving as prey (Hausmann, 1978). In Coleps hirtus the toxins of toxicysts include phytanic acid as well as saturated and unsaturated fatty acids (Buonanno et al., 2014).

\section{(2) G-protein-coupled receptors in ciliates?}

In metazoa, many cell surface receptor-ligand interactions transmitt intracellular signals via heterotrimeric GTP-binding proteins (G-proteins) composed of a dynamically interacting $\alpha$-subunit that associates reversibly with the $\beta / \gamma$-subunits. Thus, trimeric G-protein-coupled receptors (GPCRs), characterised by seven transmembrane domains, may be important as a first step in signal transduction. Depending on the type of GPCR activated and the $\alpha$-isoform associated with it, widely different signalling cascades can be activated in higher eukaryotes (Oldham \& Hamm, 2008). Trimeric G-proteins occur not only at the cell membrane, but also regulate vesicle trafficking in the Golgi apparatus (Jamora et al., 1997; Michaelson et al., 2002; Coria et al., 2014). The $\alpha$-subunits are typically ADP-ribosylated by bacterial toxins, such as by Pertussis or Cholera toxin. Among the signalling pathways following GPCR activation are widely different chemical or physical signalling steps, ranging from $\mathrm{PIns}_{2}$ cleavage by PLC to the activation of specific proteins, including some ion channels. $\mathrm{PInsP}_{2}$ is cleaved to $\mathrm{InsP}_{3}$ and DAG. Either compound can activate further signalling steps, either by $\mathrm{Ca}^{2+}$ mobilisation from stores endowed with $\mathrm{IP}_{3} \mathrm{Rs}_{\mathrm{s}}$ or by phosphorylation of specific proteins by DAG-activated PKC.

Trimeric G-proteins are also assumed to be involved in chemosensory transduction in ciliates where, however, they have not been identified unambiguously at a molecular level as yet, as outlined below. Therefore, currently Dictyostelium discoideum is the only protozoan for which trimeric G-proteins and GPCRs are well established (Manahan etal., 2004). Here, the activation cascade following extracellular cAMP binding is paradigmatic for GPCR activation, followed by activation of Ras and cytosolic adenylate cyclase (Jin, 2011). The current situation in ciliates is rather intriguing. Based on the following observations, clarification will require combining work with molecular biology and biochemistry, including standard effects of Cholera and Pertussis toxins, and thio-derivatives of GTP and GDP etc.

The autocrine mitogenic effect of Euplotes raikovi pheromone activation is assumed to involve a trimeric G-protein, based on the effects of Cholera toxin and $\gamma$-thio-GTP (with an enduring activation effect), as well as on recognition of a protein of appropriate size $(55 \mathrm{kDa})$, by antibodies specific for conserved regions of metazoan $\alpha$-subunits (Ortenzi et al., 2000). Similarly, evidence for the occurrence of trimeric G-proteins in Paramecium tetraurelia has been postulated from Western blots using antibodies against established subunits, from binding of GTP- $\gamma-\mathrm{S}$ and effects of stimulatory or inhibitory derivatives of GTP or of GDP as well as from ADP-ribosylation of a putative $\alpha$-subunit by Pertussis toxin (De Ondarza etal., 2003). Here, interference with trimeric G-protein function reportedly affected swimming behaviour due to effects on $\mathrm{Ca}^{2+}$-influx channels. In Tetrahymena thermophila, sequences similar to established genomic sequences of GPCR from other organisms have been used to prepare constructs for functional interference (Lampert, Coleman \& Hennessey, 2011). Again $\mathrm{Ca}^{2+}$ conductivity, and consequently swimming behaviour, was affected.

Irritatingly comparative genomic and bioinformatics analyses were unable to identify any of the G-protein subunits in ciliates, whereas a GPCR-related protein has been found in some Alveolata (Bradford et al., 2013). Specifically in ciliates, a cAMP receptor of a type restricted to invertebrates is closely related to a rhodopsin-type GPGR (Krishnan et al., 2012), but any function in signal transfer remains open. It remains enigmatic what the presence of a GPCR in the absence of trimeric G-proteins (or, at least, of a catalytic $\alpha$-subunit) would signify. Alternatively, signalling by a GPCR in a mode independent of trimeric G-proteins has been proposed (Anantharaman et al., 2011), or identification may be obscured by the notorious lack of similarity between GPCRs of different sources (Lima et al., 2014).

In Paramecium primaurelia an N-methyl-D-aspartate (NMDA)-like receptor has been envisaged (Ramoino et al., 2006, 2014). If present in ciliates this channel-type receptor could also be coupled to GPCRs and its activation would be interesting with regard to the positive chemotactic effect of glutamate by which its activity is modulated (Zito \& Scheuss, 2009). From the Paramecium database, partial sequences have been retrieved showing some similarity to important domains and to potentially associated proteins (Ramoino et al., 2014). However, in contrast to expectations (Ramoino et al., 2006) no sequences could be found matching with ionotropic glutamate receptor family members. Again, this would be compatible with the low level of similarity among members of the GPCR superfamily (Lima et al., 2014).

Any concise molecular details about signalling via trimeric G-proteins (or its $\alpha$-subunit) and GPCRs remain to be scrutinised. A stringent combination of experimental approaches and computer modelling would be particularly helpful, as has been used with Dictyostelium discoideum (Jin, 2011). 
PKC in higher eukaryotes is known to be activated by DAG in consequence of the activation of trimeric G-proteins. In Paramecium tetraurelia, partial sequences of a potential substrate (Hinrichsen \& Blackshear, 1993) could not be attributed to any PKC function or any underlying molecule. Only recent phosphoproteomic analyses with $T$. thermophila indicated the presence of different PKG paralogs (Tian et al., 2014). To end such basic uncertainty with ciliates, such analyses would be promising when combined with expression studies.

If it occurs in ciliates, a $\mathrm{Ca}^{2+} /$ polyvalent cation-sensing receptor would also be a candidate for interaction with a GPCR, as for most such receptors in metazoans (Riccardi \& Kemp, 2012). Paramecium tetraurelia reacts to polycations, such as AED, by $\left[\mathrm{Ca}^{2+}\right]_{\mathrm{i}}$ increase (Klauke \& Plattner, 1997) and massive exocytosis (Plattner etal., 1984, 1985). Similarly Tetrahymena spp. release mucocysts when exposed to cationic components. The secretatogue effect of AED in Paramecium may be comparable to that of compound 48/80 in mast cells, a condensation product from N-methyl-p-methoxyphenethylamine and formaldehyde, and of other cationic-amphiphilic drugs which all act as pseudo-allergens. These are currently assumed to use the machinery of G-protein-coupled receptors or parts thereof (Seifert, 2015). Nevertheless, effects of polycations could also involve polyvalent cation-sensing receptors of a type that is independent of trimeric G-proteins (Bockaert \& Pin, 1999) or some unrelated effects on plasmalemmal cation channels (Williams, 1997). This aspect of signalling in ciliates also remains open at present.

\section{GRAVIPERGEPTION AND GRAVIKINESIS/GRAVITAXIS}

Early spaceflight experiments revealed the sensitivity of mammalian cells in culture to 'weightlessness', i.e. microgravity (Cogoli, Tschopp \& Fuchs-Bislin, 1984). Since then unicellular models have frequently been used for such experiments. With ciliates, results are interpreted on the basis of polar mechanosensitive transduction (Machemer, $1988 b, 2014)$, based on electrophysiological recordings in mechanosensation experiments with metazoans (Gillespie \& Walker, 2001).

As outlined in this section, ciliates can perceive their orientation in space depending on the gravity field (graviperception). Gravity perception results in graviresponses which include gravikinesis (increased motor activity) and graviorientation, jointly also designated as gravitaxis/ geotaxis. This can be explained on the basis of gradual polarity of underlying ionic conductances, as outlined below.

Ciliates are able to perceive the earth's gravity field (Machemer \& Teunis, 1996; Hemmersbach \& Bräucker, 2002; Hemmersbach \& Braun, 2006; Machemer, 2014). Only genera with statocyst-like organelles, such as Loxodes (Bräucker, Machemer-Röhnisch \& Machemer, 1994; Hemmersbach etal., 1998), display positive gravitaxis, i.e. they swim downwards. This is in contrast to most other genera (Hemmersbach etal., 1998), such as Bursaria (Krause \& Bräucker, 2009), Didinium (Bräucker et al., 1994), Paramecium (Bräucker et al., 1994; Roberts, 2010; Machemer, 2014), Stylonychia (Krause, Bräucker \& Hemmersbach, 2010) and Tetrahymena (Mogami etal., 2004) which all swim upwards. Gravity perception by Loxodes is due to an intracellular receptor system involving a structure with density >1.0. Analysis of systems with negative gravitaxis was much more of a challenge. Analyses included electrophysiological recording of very weak signals; first indications of orientation-dependent sensitivity of ciliates to the gravity field came from membrane potential recordings in Paramecium caudatum (Machemer etal., 1998; Gebauer, Watzke \& Machemer, 1999). Such studies have since been complemented by micro- and hypergravity experiments.

In cells with negative gravitaxis the cytoplasm exerts pressure onto sensors that are arranged in the cell membrane along a polar gradient. These are depolarising mechanosensitive $\mathrm{Ca}^{2+}$ channels and hyperpolarising mechanosensitive $\mathrm{K}^{+}$channels enriched in the anterior and the posterior half of the cell (Section III), respectively, as analysed in the context of gravikinesis (Machemer \& Teunis, 1996; Krause \& Bräucker, 2009; Krause etal., 2010; Machemer, 2014). Downward movement activates anteriorly enriched depolarisation channels which entails reorientation of the cells in the upward direction, essentially according to the principles described for behavioural reactions (Section III). Increased upward movement is induced and supported by hyperpolarisation due to activation of hyperpolarising channels in the posterior part of the cell. One prerequisite to activation of mechano-channels is that the cytoplasm has a higher density than the surrounding medium. Since the difference is usually very small, the gating energy is only slightly above the thermal noise and, thus, provides only small voltage changes recordable during reorientation of the cells in the gravity field (Machemer etal., 1998; Gebauer etal., 1999; Hemmersbach \& Braun, 2006; Machemer, 2014). Support by amplifying structures, such as actin filaments, appears crucial. Concomitantly, current models show such channels in association with the cortical cytoskeleton, specifically with filamentous (F-)actin (Machemer, 2014). For ciliates this is a challenging view, considering that cortical actin has been under considerable debate until quite recently. Support comes from the facts that (i) mechano-channels are consistently considered associated with the cytoskeleton, notably F-actin, in other systems (Lapatsina etal., 2012), this association being mutually interdependent (Kuipers, Middelbeek \& van Leeuwen, 2012); (ii) special cortical isoforms, e.g. PtAct4, have been identified in P. tetraurelia (Sehring et al., 2010), and (iii) subplasmalemmal localisation of actin was shown by immuno-electron microscopy (Kissmehl et al., 2004). Therefore, in principle, F-actin associated with the cell membrane is available for amplifying gravity signals.

In summary, during negative gravikinesis an omnipresent physical stimulus is constitutively transduced into an 
electrophysiological signal. This in turn, according to a brief note, results in a chemical signal involving the formation of cAMP; conversely manipulation of the cAMP level affects gravikinesis (Hemmersbach etal., 2002). This is in line with the well-documented observation that, in Paramecium tetraurelia, cAMP formation is under positive control of hyperpolarising $\mathrm{K}^{+}$conductances (Bonini et al., 1986; Schultz \& Schönborn, 1994). See Section III.

\section{METABOLIC ASPEGTS OF SIGNALLING IN GILIATES}

As generally known, all cells rapidly consume and regenerate ATP as their main energy fuel, total turnover taking $\sim 1$ min or fractions thereof. There are few occasions to study such aspects in detail. During synchronous trichocyst exocytosis in Paramecium tetraurelia we registered a significant decay in ATP concentration within seconds (Vilmart-Seuwen et al., 1986). This enables us to dissect this phenomenon and pinpoint more specifically ATP-consuming steps.

This section can be summarised as outlined in Fig. 9. In the context of the ATP decay reported during synchronous trichocyst exocytosis stimulation (Vilmart-Seuwen etal., 1986), essentially eight facets of ATP/energy consumption are discussed, but only a few are elaborated as possibly relevant in quantitative terms.

ATP is present in Paramecium tetraurelia cells in a concentration of $\sim 1 \mathrm{mM}$, as in mammalian cells (Balaban, 2009). Within a few seconds of trichocyst exocytosis stimulation, [ATP] decays significantly (Vilmart-Seuwen et al., 1986). Here we discuss possible sources of ATP consumption. Theoretically, one is the termination of $\mathrm{Ca}^{2+}$ signalling by activation of $\mathrm{Ca}^{2+}$-ATPases/pumps. Activation of the SNARE-specific chaperone NSF, a triple A-ATPase (AAA-ATPase) (Whiteheart, Schraw \& Matveeva, 2001) for the assembly and/or disassembly of SNAREs at membrane fusion sites, e.g. trichocyst exocytosis sites, will also require ATP. This AAA-ATPase function is different from the P-type ATPase activities that hydrolyse ATP for sequestration of $\mathrm{Ca}^{2+}$ into stores and extrusion from the cell. Moreover, in Paramecium tetraurelia, massive exocytosis induction is superimposed on increased ciliary activity that will require ATP for the formation of second messengers and for the activation of the motor protein, dynein. $\mathrm{H}^{+}$-ATPase/pump-coupled $\mathrm{H}^{+} / \mathrm{X}^{+} / \mathrm{Ca}^{2+}$ antiporter activity may be an additional route of indirect ATP consumption (secondary active transport), as has been suggested for the activity of the contractile vacuole complex in Paramecium tetraurelia (Section VI). The physicochemical disequilibrium between $\left[\mathrm{Ca}^{2+}\right]$ and $\left[\mathrm{PO}_{4}{ }^{3-}\right]$ during a swift cortical $\left[\mathrm{Ca}^{2+}\right]$ increase will also be discussed below. Finally we ask how excessive ATP consumption occurring in ciliates during synchronous exocytosis can be compensated. In this context, a dramatic event is the reversible dephosphorylation of a $63 \mathrm{kDa}$ phosphoprotein that has been identified as phosphoglucomutase, a key enzyme for access to the glycolytic pathway.

\section{(1) $\mathrm{Ca}^{2+}$-flux, ATP decay and ATP recovery during synchronous trichocyst exocytosis}

During AED-induced synchronous trichocyst exocytosis in $P$. tetraurelia, ATP decays significantly by $\sim 20 \%$ (averaged from different strains), from between 1.1 and $1.25 \mathrm{mM}$ (Matt, Bilinski \& Plattner, 1978; Lumpert, Kersken \& Plattner, 1990), within $\sim 5 \mathrm{~s}$. ATP content recovers, depending on the strain, during the following $\sim 20-30$ s (Vilmart-Seuwen etal., 1986; Plattner \& Kissmehl, 2005). Considering a turnover time of ATP of $1-2 \mathrm{~min}$ in mammalian cells under steady-state conditions (Skog, Tribukait \& Sundius, 1982; Jauker, Lades \& Nowack, 1986), the decay of [ATP] by $\sim 20 \%$ within $5 \mathrm{~s}$ is difficult to explain. This dramatic decay would appear more feasible if the turnover time was much shorter. In fact, in Tetrahymena thermophila this has been estimated as $2 \mathrm{~s}$ (Jauker etal., 1986), although this is probably an underestimation. A realistic ATP turnover time in Paramecium tetraurelia may be between 10 and $30 \mathrm{~s}$, depending on the strain, as can be estimated from the ATP decay measured during the first second of AED stimulation (Vilmart-Seuwen etal., 1986) in a wildtype (7S: $30 \mathrm{~s})$ and a closely related strain (K401: $10 \mathrm{~s}$ ), respectively. Such a turnover time would be compatible with the reestablishment of $[\mathrm{ATP}]$ in Paramecium tetraurelia within $\leq 30 \mathrm{~s}$ after massive synchronous exocytosis (Vilmart-Seuwen et al., 1986).

To what extent can ATP hydrolysis be accounted for by $\mathrm{Ca}^{2+}$-ATPase/pump activity and how does this compare with the $\mathrm{Ca}^{2+}$ leakage rate? Paramecium tetraurelia possesses a very high $\mathrm{Ca}^{2+}$ leakage rate under steady-state conditions. In unstimulated cells this is $\sim 30$ pmole $\mathrm{Ca}^{2+}$ per $10^{3}$ cells over 5 min (Kerboeuf \& Cohen, 1990). Considering Avogadro's number $\left(6 \times 10^{23}\right)$ this corresponds to $3 \times 10^{8} \mathrm{Ca}^{2+}$ ions per cell in $5 \mathrm{~s}$, the time interval after which $[\mathrm{ATP}]$ is seen to increase again. From the volume of one Paramecium tetraurelia cell, i.e. $0.73 \times 10^{-10} 1$ (Erxleben et al., 1997) and an [ATP] of $1.2 \mathrm{mM}\left(7.2 \times 10^{20}\right.$ molecules $\left.^{-1}\right)$, a cell contains a total of $5.6 \times 10^{10}$ ATP molecules under steady-state conditions. Considering a leakage rate of $0.6 \times 10^{8} \mathrm{Ca}^{2+} \mathrm{s}^{-1}$, the ATP supply would suffice to counteract leakage for $\sim 15 \mathrm{~min}$ if $\mathrm{Ca}^{2+} /$ ATP efficiency were $1: 1$. Since a ratio of $1: 3$ is more realistic, the ATP supply would be exhausted after $5 \mathrm{~min}$ when compensating for $\mathrm{Ca}^{2+}$ leakage if not counterbalanced and disregarding ongoing ATP synthesis. Even though for a very restricted time after AED stimulation, the actual $\mathrm{Ca}^{2+}$ influx rate is $\sim 3$ times higher than during steady state (Kerboeuf \& Cohen, 1990), ATP expenditure for reestablishment of $\left[\mathrm{Ca}^{2+}\right]$ homeostasis can only partially explain the [ATP] decay observed, although it cannot be fully neglected.

Based on the following pilot calculations $\mathrm{Ca}^{2+}$ flux data may be compatible with a relatively low ATP consumption for primary and secondary active $\mathrm{Ca}^{2+}$ transport. Can $\mathrm{Ca}^{2+}$-ATPases/pumps localised to alveolar sacs (Hauser etal., 2000) and to the plasma membrane (Wright \& Van Houten, 1990; Elwess \& Van Houten, 1997), i.e. SERCA and PMCA, be significant $\mathrm{Ca}^{2+}$ regulators over short periods? Interestingly the half-time for refilling of alveolar sacs (with SERCA activity) has been experimentally verified 


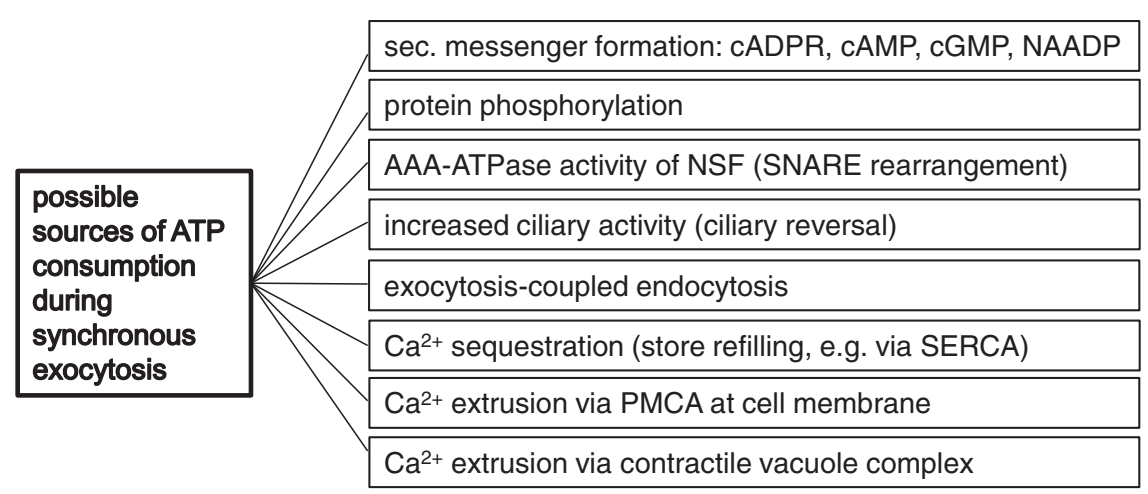

Fig. 9. Processes responsible for the consumption of ATP observed in Paramecium spp. cells during synchronous exocytosis stimulation. Pilot calculations reveal that the relative contribution of some aspects to overall ATP consumption must be very low (e.g. second messenger formation, NSF activity), in contrast to some other processes. This is likely to include mainly $\mathrm{Ca}^{2+}$ extrusion via secondary active transport (ion exchangers presumably in the contractile vacuole complex), ciliary reversal superimposed on trichocyst release and kinetic aspects of the interference of $\mathrm{Ca}^{2+}$, phosphate and adenine nucleotides. See Appendix (Section XIX) for definitions of abbreviations.

by three widely different methods, in situ and in vitro, as $65 \mathrm{~min}$ (Mohamed et al., 2003). Assuming that PMCA activity in the cell membrane (occupying about the same area as SERCA in alveolar sacs) has about the same activity, the $\mathrm{Ca}^{2+}$-pump acivity would be rather small in relation to the $\mathrm{Ca}^{2+}$ load to explain the swift consumption of ATP within $5 \mathrm{~s}$, even if one considers additional SERCA activity in the endoplasmic reticulum. Therefore, a much more rapid and efficient mechanism must be taken into account for the reestablishment of $\left[\mathrm{Ca}^{2+}\right]$ homeostasis. Rather than primary active transport, secondary active transport appears to be the dominant $\left[\mathrm{Ca}^{2+}\right]$ regulator and ATP consumer. This can be extrapolated from the substantial release of $\mathrm{Ca}^{2+}$ by the contractile vacuole (Stock etal., 2002) and the significant retardation effect of the $\mathrm{H}^{+}$-ATPase inhibitor, concanamycin $\mathrm{B}$, on the reestablishment of $\left[\mathrm{Ca}^{2+}\right]_{i}$ after strong exocytosis stimulation (Plattner etal., 2012). Under steady-state conditions the contractile vacuole complex can, thus, export its excess of $\mathrm{Ca}^{2+}$ caused by steady-state leakage within $\sim 5.9 \mathrm{~min}$ (Ladenburger et al., 2006). Interestingly this is comparable to the ATP supply that would suffice to counterbalance $\mathrm{Ca}^{2+}$ permeation, as discussed above. Increased $\left[\mathrm{Ca}^{2+}\right]_{\mathrm{i}}$ can be assumed to activate secondary active extrusion mechanisms, such as $\mathrm{H}^{+} / \mathrm{X}^{+} / \mathrm{Ca}^{2+}$ exchangers; although not yet explored in ciliates there are stringent reasons to assume their existence (Section XIV). Another rapid and powerful $\mathrm{Ca}^{2+}$ regulator after stimulation is the rapid binding of $\mathrm{Ca}^{2+}$ to immobile buffers, such as centrin (Sehring etal., 2009) - independent of ATP. Our calculations consider acute roles of direct or indirect active $\mathrm{Ca}^{2+}$ transport which, surprisingly, can account only for a fraction of the ATP consumed during synchronous exocytosis.

\section{(2) ATP decay - unlikely due to second messenger formation, SNARE rearrangement or exocytosis-coupled endocytosis}

Some signalling steps depend on metabolites from which 'metabolic' second messengers are generated. Formation of
cAMP depends on ATP, that of cGMP on GTP (formed from ATP by GDP transphosphorylation), $\mathrm{InsP}_{3}$ on $\mathrm{PInsP}_{2}$ and finally cADPR and NAADP formation depend on $\mathrm{NAD}(\mathrm{P})$ (NAD and NADP being $\mathrm{H}^{+}$acceptors in bioenergetic processes). However, second messenger formation is unlikely to cause remarkable ATP consumption because of their low concentration, usually nano- to (sub)micromolar. For instance, a $\mathrm{K}_{\mathrm{d}}=3.3 \mathrm{nM}$ has been determined for NAADP in Paramecium tetraurelia homogenates (Plattner et al., 2012).

Could some of the ATP consumption measured during synchronous trichocyst exocytosis be due to NSF activity? ATP-driven disassembly of SNARE complexes consumes ATP and up to six SNARE complexes are usually assumed to occur per exocytosis site, based on data from mammalian cells (Plattner, 2010b). Consider again the availability of $5.6 \times 10^{10}$ ATP molecules per cell (Section XII.1). The 50 ATP molecules required to disentangle a trans-SNARE complex (Cipriano etal., 2013), the six SNAREs per exocytosis site, and the $\sim 1000$ trichocyst release sites (Plattner et al., 1985) results in a total of $3 \times 10^{5}$ ATP molecules consumed per cell for the AAA-ATPase function of NSF. The percentage of ATP consumed during synchronous trichocyst exocytosis, therefore, would be only $\sim 0.6 \times 10^{-3} \%$ of the available store. Even assuming 10 times higher numbers of SNAREs per site (Sieber et al., 2007) NSF activity could not explain the ATP consumption actually observed during synchronous trichocyst exocytosis.

Considerable ATP consumption has been assigned to recycling of transmitter vesicles in hippocampal synapses (Rangaraju, Calloway \& Ryan, 2014), but this is probably due to steps beyond membrane resealing. In Paramecium tetraurelia, retrieval of trichocyst 'ghosts' follows synchronous exocytosis after $0.35 \mathrm{~s}$ (Knoll et al., 1991a), but takes much longer than any other process envisaged here (Plattner, Knoll \& Pape, 1993). Also [ATP] decay observed in exocytosis-incompetent strains (Vilmart-Seuwen et al., 1986) makes this an unlikely explanation for the rapid, transient consumption ATP in the course of exocytosis stimulation. 


\section{(3) ATP decay due to superimposed ciliary reversal?}

Could ATP be consumed extensively in the course of ciliary reversal which always accompanies exocytosis stimulation as a consequence of $\mathrm{Ca}^{2+}$ spill-over into cilia (Plattner etal., 1984, 1985; Section III.4)? Data are available for ATP consumption during normal swimming in Paramecium sp., (Gueron \& Levit-Gurevich, 1999). The active stroke of one cilium consumes $9 \times 10^{-16} \mathrm{~J}$, the recovery stroke $2 \times 10^{-16} \mathrm{~J}$, giving a total of $11 \times 10^{-16} \mathrm{~J}$ consumed per ciliary activity cycle. According to the general rule that $44 \mathrm{~kJ}$ are provided per mole ATP and considering Avogadro's number, $1 \mathrm{~J}$ would thus be represented by $0.14 \times 10^{20}$ ATP molecules. Therefore, one beat cycle would consume $0.15 \times 10^{5}$ ATP molecules per cilium. Assuming a cycle of $20 \mathrm{~Hz}$ and the involvement of $\sim 3000$ cilia per cell [derived from the number of unit fields, i.e. kinetids (Erxleben et al., 1997)], $7.5 \times 10^{5}$ ciliary beats occur per cell during the $5 \mathrm{~s}$ of ATP decay. Therefore, during this time, for normal beat activity, $1.1 \times 10^{10}$ ATP molecules are consumed, or $0.22 \times 10^{10} \mathrm{~s}^{-1}$ which is equivalent to $4 \%$ of the ATP available. This is more than previously estimated for the smaller Tetrahymena thermophila cells which have been estimated to consume $\sim 1 \%$ of their energy supply for normal swimming activity (Jauker et al., 1986).

To explain the considerable $[\mathrm{ATP}]$ decay within $5 \mathrm{~s}$ of AED stimulation one should also take into account the occurrence of ciliary reversal which involves intense rotation of the cell. For the following reasons this may require more ATP consumption than normal swimming. First, recall that the active stroke consumes considerably more energy than the recovery stroke and this may also hold for permanent rotation. Second, this expectation is in line with increasing ciliary activity in parallel to $\left[\mathrm{Ca}^{2+}\right]$ increase, as recorded with permeabilised cells (Nakaoka et al., 1984); consider that $\left[\mathrm{Ca}^{2+}\right]$ in cilia also increases considerably during AED stimulation (Husser et al., 2004). Third, support comes from experiments with $P$. tetraurelia mutants where, in response to AED stimulation, ciliary reversal takes place even in exocytosis-incompetent strains (Plattner etal., 1984, 1985) and they also display ATP decay upon (mock)stimulation with AED (Vilmart-Seuwen et al., 1986). Interestingly, 'pawn' mutants such as strain $d 4-500 r$ (which cannot react to depolarisation by ciliary reversal because of the absence of $\mathrm{Ca}^{2+}$ influx via defective voltage-dependent $\mathrm{Ca}^{2+}$ channels) not only perform ciliary reversal upon (mock)stimulation with AED, but also display the usual [ATP] decay (Vilmart-Seuwen et al., 1986; Müller et al., 2002). As discussed in Section III.4, this is due to $\mathrm{Ca}^{2+}$ spillover from the soma into the cilia. In summary, ciliary reversal can be considered to represent an event of extra ATP consumption, assuming that it is increased by intense rotation activity during reversal. Still some additional mechanism may be involved.

\section{(4) Effects of massive exocytosis on ATP synthesis}

Some of the $[\mathrm{ATP}]$ decay seen upon exocytosis stimulation may be due to inhibited ATP synthesis because of kinetic considerations resulting from the mass action law. During AED stimulation the dynamic equilibrium between ADP, ATP and $\mathrm{PO}_{4}{ }^{3-}$ will be affected by increased $\left[\mathrm{Ca}^{2+}\right]$. When calculated from the contribution of the two $\mathrm{Ca}^{2+}$ sources, release from alveolar sacs and influx, and neglecting downregulation phenomena, the global $\left[\mathrm{Ca}^{2+}\right]$ would increase to $0.325 \mathrm{mM}$ (Hardt \& Plattner, 2000). Still higher values can theoretically be extrapolated for cortical cell layers within short periods, again neglecting rapid downregulation (e.g. by cortical CaBPs; see above and Sections III and V). Massive $\left[\mathrm{Ca}^{2+}\right]_{\mathrm{i}}$ increase has to be counteracted not only because of toxic effects of $\mathrm{Ca}^{2+}$, but also because $\left[\mathrm{Ca}^{2+}\right]$ could come close to, or even surpass the solubility of $\left[\mathrm{PO}_{4}{ }^{3-}\right]$ in the cells. Excessive $\left[\mathrm{Ca}^{2+}\right]$ increase will bind $\mathrm{PO}_{4}{ }^{3-}$ and, if not transiently forming highly insoluble calcium phosphates (see http://butane.chem.uiuc.edu/pshapley/ GenChem2/Tables.html), it could reduce the availability of phosphate for the ADP $\rightarrow$ ATP rephosphorylation processes. Considering the rapid turnover of ATP (Section XII.1) the cells may be energetically compromised by this kinetic effect before the excess of $\mathrm{Ca}^{2+}$ is downregulated.

Therefore, the kinetics of ATP generation is crucial. In the cell cortex, the availability of ATP is expected to be restricted within narrow spaces, such as cilia, and the narrow space between plasmalemma and alveolar sacs. In large protozoan species, to improve availability of dissolved $\mathrm{O}_{2}$, mitochondria are frequently enriched in cortical layers (Fenchel, 2014). This has been observed in P. tetraurelia (H. Plattner, unpublished observation). In Paramecium tetraurelia, when analysed in vitro, [ATP] within cilia is only $\sim 0.15 \mathrm{mM}$ and, thus, only $\sim 12-15 \%$ of the whole-cell value (Noguchi, Sawada \& Akazawa, 2001). For sufficient energy supply in vitro, phosphoarginine has to be provided at concentrations of $\sim 0.4 \mathrm{mM}$ (Noguchi etal., 2001). This serves for the rephosphorylation of ADP to ATP, the substrate of the dynein ATPase that drives ciliary beating, and involves phosphoarginine provided by a ciliary arginine kinase which in Tetrahymena pyriformis is $40 \mathrm{kDa}$ in size (Michibata et al., 2014). Generally, phosphagenes help to maintain the ATP level in narrow spaces, as discussed previously (Plattner \& Kissmehl, 2005). In muscle, compensation mechanisms include phosphocreatine (in mammals) or phosphoarginine in crustaceans as a store of sufficient free enthalpy. In ciliates rapid restoration of energy supply can, thus, be achieved for the ciliary subcompartment (Noguchi et al., 2001) and most likely also in the narrow space between the plasmalemma and alveolar sac. This mechanism requires positioning of some key enzymes precisely at relevant cortical regions (see below). The compensating effects of the phosphagenes may not suffice sufficiently to counteract the abrupt [ATP] decay in narrow spaces and the ciliate cell therefore activates glycolytic substrates, as follows.

How can a Paramecium cell avoid being compromised by such ATP decay, particularly considering its rapid turnover? In Paramecium tetraurelia, a phosphoprotein of $63 \mathrm{kDa}$ (pp63) is dephosphorylated during trichocyst exocytosis and was, therefore, called 'parafusin' (Gilligan \& Satir, 1982). Its dephosphorylation actually occurs during the $80 \mathrm{~ms}$ required 
for AED-induced synchronous exocytosis (Höhne-Zell et al., 1992), followed by rephosphorylation during $\sim 10-30 \mathrm{~s}$ (Zieseniss \& Plattner, 1985). When the 'non-discharge' mutant strain $n d 9-28^{\circ} \mathrm{C}$ (Gilligan \& Satir, 1982) or any of the other exocytosis-incompetent strains (Zieseniss \& Plattner, 1985) are (mock)stimulated for exocytosis, parafusin/pp63 is not dephosphorylated. [Recall that exocytosis-incompetent strains generate the same $\mathrm{Ca}^{2+}$ transient as wildtype cells (Klauke \& Plattner, 1997) and the same ciliary response (Plattner et al., 1984, 1985)]. Evidently the $\mathrm{Ca}^{2+}$ signal cannot be transmitted to the defective fusion machinery and none of the subsequent steps (membrane resealing, detachment of ghosts, i.e. surface restructuring) take place.

Yet parafusin/pp63 has been identified as phosphoglucomutase (Hauser et al., 1997; Müller et al., 2002). According to MALDI analysis, the molecule is phosphorylated on Ser and Thr residues (Kussmann et al., 1999). In vitro it is dephosphorylated by calcineurin/PP2B (Kissmehl et al., 1996), cloned and characterised also in Paramecium tetraurelia (Fraga et al., 2010). It is rephosphorylated by a $\mathrm{Ca}^{2+}$-inhibited casein kinase, also known in Paramecium tetraurelia (Vetter etal., 2003). Thus, a $\mathrm{Ca}^{2+}$ signal may induce and maintain the dephosphorylated state and cause activation of phosphoglucomutase activity to funnel substrate into the glycolytic pathway within the narrow subplasmalemmal space where it is localised (Plattner \& Kissmehl, 2005). This activation is assumed to depend on the desorption of dephospho-parafusin/(p)p63 from structures to which it is normally bound in its phosphorylated form. Soluble enzymes are known to have frequently a higher activity state in the free form, e.g. due to more enzyme/substrate 'encounters' and lack of steric constraints.

Thus, Paramecium tetraurelia cells reversibly dephosphorylate parafusin/pp63 for $<30 \mathrm{~s}$ (Zieseniss \& Plattner, 1985 ) and regain their normal ATP level within $\sim 20-30 \mathrm{~s}$ (Vilmart-Seuwen etal., 1986) after AED stimulation. Remarkably, restoration of the ATP pool is greatly retarded in cells unable to dephosphorylate parafusin/pp63 (Müller et al., 2002; Plattner \& Kissmehl, 2005). Considering an ATP turnover time of $<30 \mathrm{~s}$ (see above) the viability of such cells could be compromised [although in knockout experiments with Tetrahymena thermophila, mucocyst exocytosis can still be performed (Chilcoat \& Turkewitz, 1997)]. In fact, $\mathrm{Ca}^{2+}$ homeostasis is compromised in yeast cells after disruption of the phosphoglucomutase gene (Fu et al., 2000). Activation of phosphoglucomutase can increase NADH and ATP production in cytosol and mitochondria and, thus, avoid compromising the cells energetically during massive exocytosis stimulation.

In addition, in Paramecium tetraurelia ATP consumption will in part be counteracted by stimulation of mitochondria. Upon induction of synchronous trichocyst exocytosis, $\left[\mathrm{Ca}^{2+}\right]$ instantaneously increases in mitochondria (Hardt \& Plattner, 2000). This requires a uniporter of a type detected in Tetrahymena thermophila (Bick, Calvo \& Mootha, 2012). Comparison of signals measured in mitochondria for whole and free calcium $\mathrm{Ca}^{2+}$ using X-ray microanalysis (Hardt \& Plattner, 2000) and fluorochrome analysis (Plattner etal.,
2012), respectively, reveals that only a small fraction of $\mathrm{Ca}^{2+}$ entering a mitochondrium is retained in the organelle. Consider that in muscle the entire cascade of oxidative phosphorylation (Glancy etal., 2013) as well as ATP synthase activity is activated (Balaban, 2009) and only some of the mitochondrial matrix dehydrogenases (Denton, 2009). Therefore, a similar ATP-restoring effect can be reasonably expected to accompany exocytosis in Paramecium spp. Additional components of a mitochondrial $\mathrm{Ca}^{2+}$ uniporter have been identified in mammalian cells (Sancak et al., 2013). Here, subunits type 1 and 2 of mitochondrial calcium uptake (MICU), the pore-forming subunit mitochondrial calcium uniporter (MCU) in conjunction with the MCU regulator, essential MCU regulator (EMRE), mediate interaction of subunits to form a functional channel (Kamer \& Mootha, 2014). Note that MICUs are EF-hand CaBPs. In Tetrahymena thermophila, MCU and MICU1 have been identified, in contrast to EMRE, while MICU2 was not the focus of that study (Sancak et al., 2013). Thus, important aspects are already in place to support the hypothesis that ATP decay is immediately counteracted by resynthesis, also in mitochondria.

In summary there remain several possible explanations for the ATP decay seen during massive exocytosis induction. (i) Ciliary reversal (assuming that it consumes more ATP than normal forward swimming). (ii) A secondary active $\mathrm{Ca}^{2+}$ transport coupled directly or indirectly to a $\mathrm{H}^{+}$-ATPase is likely to be more important than a primary active $\mathrm{Ca}^{2+}$ transport. (iii) A swift (5s) reduction in ATP synthesis is expected for kinetic reasons, i.e. $\mathrm{Ca}^{2+} / \mathrm{PO}_{4}{ }^{3-}$ disequilibrium due to a significant cortical $\left[\mathrm{Ca}^{2+}\right]$ increase. The activation of parafusin/pp63 by dephosphorylation and the activation of mitochondrial ATP synthesis are considered able to counteract ATP decay efficiently, so that [ATP] can be restored within $\sim 20-30$ s without compromising the vitality of the cells even after massive exocytosis stimulation.

\section{FEEDBACK FROM GYTOPLASMIC FUNCTIONAL STATES, FROM MITOCHONDRIA AND FROM ENDOSYMBIONTS TO THE MAGRONUGLEUS}

Generally cells control the production of proteins according to functional needs, based on a feedback system (Fig. 10). Mucocyst biogenesis in Tetrahymena thermophila has served as a paradigm in the field (Haddad \& Turkewitz, 1997) and the underlying mechanisms may be of medical interest (Hutton, 1997). In ciliates, feedback has to reach the macronucleus, but how signalling takes place in molecular terms remains to be explored. Remarkably, in Tetrahymena thermophila, the microand macronucleus have different subsets of nucleoporins which may mediate selective transport of signals into the macronucleus (Iwamoto et al., 2009). In Paramecium tetraurelia, corresponding genes are upregulated after massive exocytosis, as is also the case after radical deciliation. A genome-wide microarray analysis of gene expression in Tetrahymena thermophila revealed the upregulation of an unexpectedly 


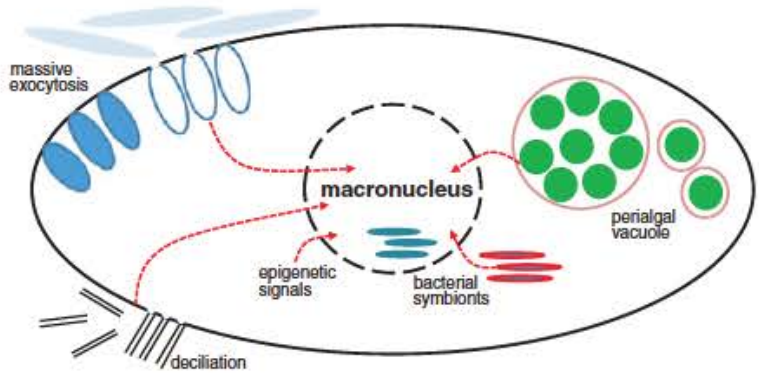

Fig. 10. A variety of signals feed back into the transcriptionally active macronucleus of ciliates. This occurs after massive exocytosis or after deciliation; other signals emerge from endosymbiotic bacteria and green algae (similarly to signals arising from mitochondria).

large number of specific genes also during conjugation (Miao et al., 2009). Endosymbionts exert a similar feedback to transcription (Fig. 10). In higher eukaryotes, nuclear feedback is particularly well established for endosymbiontderived organelles, such as mitochondria, but data for ciliates are not yet very detailed.

\section{(1) Feedback from the cytoplasm to the macronucleus}

Some signalling molecules are transferred from the cytosol into the nucleus to influence transcription. A longstanding example is the transcription factor, 'nuclear factor of activated T-cells' (NFAT), that is activated via dephosphorylation by PP2B and then activates T-lymphocytes (Baksh \& Burakoff, 2000). Interestingly this phosphatase also occurs in Paramecium tetraurelia according to evidence from cell biology (Momayezi et al., 1987), biochemistry (Kissmehl et al., 1997) and molecular biology (Fraga et al., 2010). However, nuclear signalling by calcineurin has not yet been explored in ciliates.

In ciliates, feedback from the cytosol to the transcriptionally active macronucleus does take place. This has been shown in connection with massive mucocyst release in Tetrahymena thermophila (Haddad \& Turkewitz, 1997) as well as in Paramecium tetraurelia after massive trichocyst exocytosis (Arnaiz etal., 2010). The same occurs in the course of reciliation following deciliation (Arnaiz et al., 2010). Genes encoding components of the respective organelles, which have to be synthesized de novo, are most intensely transcribed, but many others are also upregulated. Not all translation products can easily be fitted into the spectrum one would expect based on current knowledge about signalling from cilia to the nucleus in mammalian cells (Basten \& Giles, 2013) and about biosynthesis and function of cilia and of dense core-secretory organelles. Although unexplored, in ciliates, $\mathrm{PP} 2 \mathrm{~B} /$ calcineurin may play a role in organelle regeneration by activation of gene transcription. In metazoans, different specific transcription factors were identified as crucial for ciliary biogenesis and function (Choksi et al., 2014).

In higher eukaryotes nuclear actin is capable of remodelling chromatin (Kapoor \& Shen, 2014) and can regulate the transcriptional activity of RNA polymerases (Treisman,
2013). This may also be applicable to ciliates, as PtAct4 localises to the transcriptionally active macronucleus in regions adjacent to heterochromatin domains (Sehring et al., 2010). Its monomeric nuclear form (Kapoor \& Shen, 2014) would not have been detected in studies with fluorescent drugs, such as phalloidin, for which this actin paralog has no binding motifs.

\section{(2) Feedback from mitochondria and endosymbionts}

In general terms, feedback between endosymbiont and nuclear genomes is important in several regards. Early in evolution symbiosis led to the transformation of bacteria to mitochondria. With pathogenic Apicomplexa the interplay between a vestigial genome of a plastid-derived endosymbiont with mitochondria and cytosol has received attention as an example of recent evolution, with the potential for medical exploitation (Howe \& Purton, 2007). Currently the interplay between Legionella pneumophila and amoebae is also raising medical interest (Escoll etal., 2013). In Paramecium spp., bacterial endosymbionts provide a feedback to the nuclear genome. Studies about endosymbionts in ciliates may yield hints to mechanisms for establishing obligatory endosymbiosis.

\section{(a) Mitochondria}

Data about signalling between mitochondria and the macronucleus in ciliates, including Paramecium spp., are limited (Sainsard-Chanet \& Cummings, 1988; Beale \& Preer, 2008). As expected from other organisms, the biosynthesis of important enzymes depends on proper interaction between the nuclear and the mitochondrial genome. Wildtype mitochondria microinjected into Paramecium tetraurelia mutants or into related species, are degraded due to incompatibility (Sainsard-Chanet \& Cummings, 1988), thus suggesting fine-tuning between the two compartments. Remarkably, in humans, transfer of mitochondria from healthy donors, combined with in vitro fertilisation, is currently under public discussion as a means to overcome mitochondriopathies.

The mitochondrial genome of seveal ciliates, i.e. species of Euplotes, Paramecium and Tetrahymena, is now fully sequenced. How is crosstalk between the mitochondrial and the nuclear genome effected? 44 protein-coding genes are found in the mitochondrial DNA of T. pyriformis (Barbrook etal., 2010), but only 13 in man (Schon, DiMauro \& Hirano, 2012). According to mass spectrometry of mitochondria isolated from Tetrahymena thermophila, 545 of a total of 573 identified mitochondrial proteins are imported (Smith et al., 2007). In Tetrahymena thermophila, compared to man, fewer mitochondrial tRNAs (Brunk et al., 2003) and mitochondrial ribosomal RNAs are encoded by mitochondrial DNA (Barbrook et al., 2010). This is in agreement with a tendency for decreasing autonomy of mitochondria during evolution. In the future, genomics and proteomics should allow us to delve more deeply into the signalling between mitochondrial and macronuclear genomes in ciliates. 


\section{(b) Symbionts}

Among ciliates, Paramecium species are particularly liable to the symbiotic integration of bacteria, e.g. Holospora species, as obligatory symbionts in their cytoplasm or macronucleus. A periplasmic protein of $89 \mathrm{kDa}$ supports host cell invasion (Fujishima \& Kodama, 2012). This alters gene transcription and lowers stress sensitivity. Signalling processes required for the escape from phagosomes and the establishment of symbiosis may now be more easily amenable to systematic study with genetically altered Escherichia coli cells, as shown with Tetrahymena pyriformis (Siegmund et al., 2013).

Some ciliate species (e.g. Paramecium bursaria) can establish permanent symbiosis with Chlorella algae. Experimental infection revealed distinct steps (Kodama \& Fujishima, 2010): (i) phagocytosis of the algae, (ii) fusion with acidosomes, (iii) fusion with lysosomes; (iv) some of the surviving Chlorella cells bud from the phagolysosomes and, enclosed in a membrane, (v) accumulate at the cell periphery. Signals for these distinct steps, also including resistance to the fusion of stage iv-symbionts with lysosomes, remain to be elucidated. It became evident from mRNA profiling that, after subtraction of Chlorella sequences, the transcription of many proteins is selectively enhanced in symbiont-bearing P. bursaria cells (Kodama etal., 2014). The propagation of the algae is synchronised with Paramecium's cell cycle by an unknown signal (Gerashchenko, 2010).

\section{DOWNREGULATION OF THE MOST COMMON AGTIVATION MEGHANISMS}

In many cases, activation of a cell should be transient, occasionally even very short. A good example is $\mathrm{Ca}^{2+}$ (Berridge etal., 2003) of which some aspects in Paramecium spp. have been discussed in preceding sections. $\mathrm{Ca}^{2+}$ signals in particular have to be rapidly downregulated, not only to avoid toxic effects, but also to minimise energy expenditure and to restrict the signal to very local effects near precisely positioned channels (Sections I, II, VIII and XII). In all eukaryotic cells inactivation mechanisms can include feedback by downregulation of $\mathrm{Ca}^{2+}$ influx or mobilisation, by binding to CaBPs, and by sequestration or extrusion from the cell. Metabolic messengers, such as cyclic nucleotides are broken down by phosphodiesterases (few details being known for ciliates). Similarly protein phosphorylation can be reversed by phosphatases of which ciliates possess different types. Ciliates have examples of all these mechanisms, as summarised in Fig. 7 for downregulation of $\mathrm{Ca}^{2+}$ signals by primary active and possibly also by secondary active transporters.

For $\mathrm{Ca}^{2+}$ signals there are different routes of inactivation (Berridge etal., 2003). Negative feedback by the inhibitory $\mathrm{Ca}^{2+} / \mathrm{CaM}$ complex can be used to inactivate ciliary voltage-dependent $\mathrm{Ca}^{2+}$-channels during ciliary reversal (Brehm \& Eckert, 1978), as outlined in Section III.2. Binding to high-capacity/low-affinity CaBPs can also terminate a $\mathrm{Ca}^{2+}$ signal. This is achieved, for instance, by cortical centrin after massive trichocyst exocytosis stimulation (Sehring etal., 2009), but may also take place at the base of cilia to terminate ciliary $\mathrm{Ca}^{2+}$ signals (Gonda, Oami $\&$ Takahashi, 2007). Slow dissipation of $\mathrm{Ca}^{2+}$ from the low-affinity binding sites of CaBPs may subsequently allow its removal by extrusion. In Paramecium spp. this probably involves a secondary active transporter (putative $\mathrm{H}^{+} / \mathrm{Ca}^{2+}$ and/or a similar antiporter) for $\mathrm{Ca}^{2+}$ sequestration in acidic compartments, such as the contractile vacuole complex (Plattner etal., 2012; Plattner, 2013a) and periodic release via the contractile vacuole pore (Stock etal., 2002). This can be concluded from the effect of the $\mathrm{H}^{+}$-ATPase inhibitor, concanamycin B (Grønlien et al., 2002), which inhibits $\left[\mathrm{Ca}^{2+}\right]_{\mathrm{i}}$ downregulation after exocytosis stimulation (Plattner etal., 2012), as outlined in Sections VI and XII. An abundance of primary and secondary active transporters and channels have been implied by bioinformatic analysis of the genomic databases of P. tetraurelia, T. thermophila and of the fish pathogen, Ichthyophthirius multifiliis (Kumar \& Saier, 2015).

Ciliates possess primary active $\mathrm{Ca}^{2+}$ transporters, including the sarcoplasmic/endoplasmic reticulum- (SERCA-) type and the PMCA. Both are P-type ATPases, i.e. forming a phospho-intermediate. In ciliates, the kinetics of the SERCA is much slower than required for rapid $\mathrm{Ca}^{2+}$ downregulation (Mohamed etal., 2003) and the same may hold true for the PMCA (Plattner \& Klauke, 2001; Section XII). For instance, alveolar sacs are refilled with $t_{1 / 2}=65 \mathrm{~min}$ (Mohamed et al., 2003). Therefore, secondary active transport systems are probably more important (Plattner, 2014), although reliable data are still missing. In ciliates, primary active $\mathrm{Ca}^{2+}$ transport appears more appropriate for general household functions rather than for even moderately rapid $\mathrm{Ca}^{2+}$ dynamics.

Many of the signals regulating membrane trafficking involve several specific steps and, therefore, are transient. This applies, e.g. to the reversible binding of successive ligands, as is the case with small GTPases. Also the phosphorylation state of proteins may change in signalling cascades. In cilia protein phosphatase 1 (PP1) and PP2C are localised to the peripheral part of the axoneme (Momayezi etal., 1996; Grothe etal., 1998) and thus could contribute to reversing the state of phosphorylation, i.e. of activation. For instance, termination of ciliary reversal is inhibited by inhibition of PP1 activity (Klumpp etal., 1990). The importance of the dephosphorylation of dynamin - a well-established prerequisite for clathrin-mediated endocytosis in other systems - is suggested by the association of PP2B/calcineurin with parasomal sacs in Paramecium tetraurelia (Momayezi, Kissmehl \& Plattner, 2000).

The behavioural response to exogenous nucleotide triphosphates, i.e. repetitive back- and forward swimming, in Tetrahymena spp. and Paramecium spp. (Section X) is spontaneously attenuated and ended upon longer exposure time, probably by receptor adaptation (Kim et al., 1999; Hennessey, 2005). 


\section{EPIGENETIG SIGNALLING, ENGYSTMENT AND 'PROGRAMMED NUGLEAR DEATH' IN GILIATES}

Epigenetic signalling by non-DNA-encoded signals is the focus of current biomedical research, particularly since the discovery of prion proteins $(\mathrm{PrPs})$. In mammals, the normal conformation $\operatorname{PrP}^{\mathrm{c}}$ can be misfolded to $\operatorname{PrP}^{\mathrm{sc}}$ (' $\mathrm{c}$ ' and 'sc' standing for cellular and scrapie disease, respectively). The $\mathrm{PrP}^{\mathrm{sc}}$ form propagates based on epigenetic information transfer onto $\mathrm{PrP}^{\mathrm{c}}$ molecules - until the abundance of $\mathrm{PrP}^{\mathrm{sc}}$ acts as a pathogen causing brain degeneration (Prusiner, 2004). Remarkably, the concept of epigenetic information transfer is based on seminal observations with fungi and ciliates (Beisson, 2008). The regular design of the ciliate cell surface (Section I.4), its surface antigens, macronuclear differentiation etc. are all epigenetically determined, thus making ciliates attractive models (Simon \& Plattner, 2014). Phenomena thus controlled range from tailoring of the new macronuclear genome (Duharcourt, Lepère \& Meyer, 2009) when old macronuclei are disrupted ('nuclear death', see Section XV.2) and non-Mendelian expression of surface molecules (Sonneborn, 1949) to cell surface pattern formation (Frankel, 1973; Beisson, 2008) and even change of cell size and shape. The information may come from the macronucleus, the cell surface, or from soluble material (kairomones) in the medium. Several important molecular key players have recently been detected in ciliates and are discussed below in the context of epigenetic signalling.

The second aspect discussed here concerns apoptosis-like phenomena. In metazoans, apoptosis is the most prominent form of programmed cell death which is regulated by a complicated network. This includes 'DNA ladder' formation by fragmentation to nucleosome-size DNA pieces and multiples thereof, mitochondrial degeneration and participation of nuclear, mitochondrial and cytosolic proteins. This includes apoptosis-inducing factors, protease-type caspases, endonucleases, etc. (Norberg, Orrenius \& Zhivotovsky, 2010). Elements of the apoptotic machinery can be traced back to unicellular unikonts, such as choanoflagellates (Zmasek \& Godzik, 2013), although important questions remain open with all protozoa.

While ciliate cells can divide over long periods, for 'quasi-eternal' life, they can rejuvenate their macronuclei by formation of new ones from the micronucleus (corresponding to soma and germline, respectively, in metazoa). There are two ways, (i) conjugation (cell pairing with exchange of dividing micronuclei) and (ii) autogamy (internal 'fertilization' by fusion of dividing micronuclei within one cell) - each of these processes resulting in the formation of a new macronucleus per cell, serving for gene transcription. Degradation of the old macronucleus is paralleled by formation of a 'DNA ladder' and the abundance of autophagy, including mitochondria, with this respect closely resembling genuine apoptosis (Endoh \& Kobayashi, 2006). Is it justified to consider these details as sufficient criteria for programmed cell death - or rather 'programmed nuclear death'?

\section{(1) Feedback from intracellular epigenetic signals}

Regulation of surface pattern formation has to consider not only the specific localisation of components in anterior/posterior, top/bottom and left/right dimensions, but also pattern formation on a variable scale. Examples range from the polar arrangement of somatic ion channels, sites of contractile vacuole and extrusion sites of spent food vacuoles (cytoproct), arrangement of cilia for rotational movement to organisation of kinetids. All are epigenetically controlled (Beisson \& Sonneborn, 1965; Frankel, 1973; Beisson \& Jerka-Dziadosz, 1999; Beisson, 2008). Many factors cooperate, as derived from the analysis of mutants of different Paramecium and Tetrahymena species. Whole-genomic analysis of covariation in mutants or during knockout of selective components (see below) would appear feasible, considering these widely different components.

In Paramecium tetraurelia cells, surface pattern formation depends on protein phosphorylation processes (Keryer et al., 1987; Sperling et al., 1991). Another factor is the self-assembly of epiplasmins (Aubusson-Fleury etal., 2013). Alveolins, proteins with charged repeat motifs, appear relevant for regular surface pattern formation since knock-down entails disorganised cortical pattern in misshapen cells, as demonstrated with Tetrahymena thermophila (El-Haddad et al., 2013). Such effects are also observed after knock-down of other cortical components, e.g. of a cortical actin isoform, PtAct4 (Sehring etal., 2010) and a variety of SNAREs and PtCRCs (Simon \& Plattner, 2014). Since $\gamma$-tubulin and $\eta$-tubulin are required for basal body duplication in Paramecium tetraurelia (Ruiz etal., 1999, 2000) their proper positioning may be another critical factor, together with centrin, in Paramecium spp. (Ruiz etal., 2005) as well as in Tetrahymena thermophila (Stemm-Wolf et al., 2005). So far, the role of cortical actin in cortex formation has not been explored in ciliates although it is known from mammalian cells to contribute together with tubulin ( $\mathrm{Li} \&$ Gundersen, 2008). In addition to these examples many more factors may come into play. When ciliary biogenesis has been analysed in mutants by light and electron microscopy, as recently summarised by Pearson (2014), multiple basal body formation in proper arrangement depends on asymmetrically spaced scaffolds. These include transiently visible structures, such as the 'anterior left filament' of infraciliary lattice filaments (ILF) (Jerka-Dziadosz etal., 2013) and centrin isoforms -2 and -3 (Ruiz etal., 2005; Stemm-Wolf etal., 2005), together with their binding partners, e.g. members of the Sfi protein family (Stemm-Wolf, Meehl \& Winey, 2013). Transient asymmetry thus forms and determines the mutual arrangement of newly forming basal bodies (Pearson, 2014).

Epigenetic signalling observed during formation of a new macronucleus after conjugation or autogamy is a consequence of nuclear dimorphism. Transcripts from the meiotic micronucleus pair with gene sequences from the old macronucleus to generate sequences destined for the newly forming macronucleus (Duharcourt etal., 2009; Nowak etal., 2011). This process results in the elimination of precisely defined 'internal eliminated sequences' (IES). 
Originally this probably evolved as a mechanism for the elimination of transposons (Klobutcher \& Herrick, 1995; Chalker \& Yao, 2011), but later, in ciliates, this mechanism has been integrated into normal, epigenetically controlled cell development. IES elimination is executed by different transposases; in oligotrichs, such as Paramecium and Tetrahymena, it is a domesticated PiggyBack transposase (Vogt \& Mochizuki, 2013) operating at sites determined by specific nucleotide flanks. Additional proteins required for IES elimination are 'protein involved in development of the somatic genome' (Pdsg) found in Paramecium (Arambasic et al., 2014) and 'defective in excision' PtDIE5 and TtDIE5, found in P. tetraurelia and T. thermophila, respectively (Matsuda et al., 2010). Heterochromatin is formed on IES sites before excision and this depends on methylation of Lys residue 9 in histone 3 (Liu etal., 2011). End-joining of excision sites involves a ligase of type IV of the non-homologous end-joining (NHEJ) pathway (Marmignon et al., 2014).

Another example, epigenetic regulation of differential gene transcription under varying external or internal/physiological conditions, has been analysed in Tetrahymena thermophila by a combined bioinformatic and proteomic approach (Zhang et al., 2014). Covariation of some specific post-translational histone modifications, including acetylation, methylation and mono-ubiquitination, indicates molecular crosstalk.

Other signalling mechanisms are under epigenetic control. For instance, mutually exclusive expression of isoforms of variant surface antigens (vsAG, 'serotypes' recognised by antibodies) in most strains of $P$. tetraurelia ensures the presence of one single kind of vsAG (GPI-anchored proteins) per cell at any time (Preer, Preer \& Rudman, 1981; Preer et al., 1987). This is regulated by a feedback mechanism (Gilley etal., 1990; Matsuda \& Forney, 2005). When a vsAG is removed, an alternative vsAG isoform can be implanted on the cell surface - all under the control of differential transcription (Leeck \& Forney, 1996). Change of culture temperature (Antony \& Capdeville, 1989; Benwakrim etal., 1998) or application of antibodies against the specific serotype actually expressed both induce the removal of the old vsAGs and transcription of an alternative serotype protein (Juergensmeyer, 1969). The mutually exclusive expression of vsAGs in Paramecium tetraurelia depends on an epigenetic signal; only subtelomeric genes are engaged in the formation of RNAi-mediated silencing of the silent vsAG species (Baranasic et al., 2014). Thus, the considerable extent of chromosome fragmentation in these ciliate cells becomes important.

The physiological function of vsAGs and details of their regulation in ciliates remain enigmatic (Simon \& Schmidt, 2007). A signalling function in the sense of defence against predators has been proposed (Harumoto \& Miyake, 1993; Clark \& Forney, 2003). In this case, the sense, or precise function that vsAG variation remains to be answered.

Oxytrichia species are a special case where chromosome fragmentation is much more extensive than in other ciliates, followed by rearrangement of DNA during formation of a new macronucleus from a micronuclear precursor
(Nowacki \& Landweber, 2009). In Oxytrichia spp., during nuclear transformation achieved by transiently expressed macronulear RNA templates, some mutations occurring in the scnRNA can be transferred to the new macronucleus, while the micronuclear (germline) DNA is unchanged (Nowacki \& Landweber, 2009). In this case the macronuclear genome can, therefore, be called an 'epigenome'.

\section{(2) Feedback from extracellular epigenetic signals, encystment and 'programmed nuclear death'}

\section{(a) Epigenetics}

Epigenetic signals can reach some ciliates from the surrounding medium. GPI-anchored proteins, such as vsAGs, when released from the cell surface of different ciliates, can potentially provide signalling phenomena (Simon \& Kusch, 2013). Another example is chemical signalling among species by release of substances called kairomones. Tetrahymena vorax is known for its capability to adapt its oral cavity to the size of the prey available. Buhse (1967) named the responsible kairomone 'stomatin'. Note that such 'stomatins' are different from the scaffolding SPFH-family proteins (SPFH means stomatin, prohibitin, flotillin and HflK/C superfamily). Previous attempts to establish intracellular signal transduction mechanisms in $T$. vorax by inhibitor studies (Ryals, Bae \& Patterson, 1999) suffer from the widely aberrant pharmacology of ciliates (Plattner etal., 2009). The kairomone stomatin has been identified as a complex of ferrous iron and some nucleic acid catabolites (Smith-Somerville et al., 2000). Other examples are anti-predator defences in planktonic ciliates (Lass \& Spaak, 2003; Lynn, 2010), as has been detected with Euplotes octocarinatus (Kusch \& Heckmann, 1992). These cells can react to the presence of a predatory species, Lembadion bullinum, by enlargement of body size and formation of body extensions to make them less prone to engulfment. A kairomone of $31.5 \mathrm{kDa}$, called L-factor, was isolated, and after cloning of the gene, a Cys-rich protein was recombinantly expressed in E. coli (Peters-Regehr, Kusch \& Heckmann, 1997). The cDNA-derived protein was of slightly higher molecular weight, but exerted the same kairomone function as the natural L-factor, although at somewhat higher concentration $\left(10^{-10}\right.$ versus $\left.10^{-12} \mathrm{M}\right)$.

As in other eukaryotes, gene expression in ciliates can be regulated epigenetically. Many examples of such epigenetic signalling, e.g. by histone modifications, mutually exclusive expression of paralogs etc., are discussed elsewhere (Chalker etal., 2013; Simon \& Plattner, 2014).

\section{(b) Cysts}

Some ciliates, with the exception of Paramecium spp., react to different stress factors by the formation of cysts. Stress includes adverse environmental conditions, such as starvation or high cell density (Tomaru, 2002). Cysts are forms of latent life (parabiosis) with the capability of 'reanimation'. Although analysed in numerous morphological studies, biochemical 
analysis by protein profiling has begun only recently with Colpoda cucullus (Sogame etal., 2014). The expression of a heat shock protein and of specific actin isoforms is enhanced. In Euplotes encysticus, cyst formation induced by starvation and low temperature triggers the expression of a variety of proteins, in part of unknown function and in part related to gene and RNA regulation, protein degradation, oxidation resistance, stress response, transport and cytoskeletal organisation including a keratin-like protein (Chen et al., 2014).

\section{(c) Programmed nuclear death}

The final signal an old macronucleus receives after conjugation or autogamy is that for its breakdown and elimination - a process resembling apoptosis. In ciliates, 'programmed cell death' is restricted to the degradation of the old (parental) macronucleus and, therefore, should rather be designated 'programmed nuclear death'. Programmed nuclear death is accompanied by morphological features familiar from apoptotic metazoan cells, but there are also essential differences. In Tetrahymena thermophila, the macronucleus undergoes autophagy in the following steps (Akematsu, Pearlman \& Endoh, 2010): (i) loss of nuclear pores in parallel to nuclear condensation; (ii) transformation of the outer nuclear membrane into an autophagosome-like membrane, with glycosylation sites and phosphatidylseryl residues exposed to the cytosolic side; (iii) disintegration of the inner nuclear membrane; (iv) wrapping of mitochondria with a membrane to which lysosomes become attached; $(v)$ stepwise fusion of these two organelles with the modified macronucleus, and finally (vi) autophagosomal digestion of nuclear and mitochondrial contents. This morphological sequence differs from apoptosis in metazoans where nuclei become heavily fragmented. It is unclear why only macronuclei are degraded; this may be due to differential permeation properties of nuclear pores in the macro- and micronuclei (Iwamoto et al., 2009, 2015).

Marginal notes published in the context of work with protozoa provide few indications of molecular regulators of programmed nuclear death in ciliates (Srivastava et al., 2010; Smirlis \& Soteriadou, 2011). In Tetrahymena thermophila this is initiated by an apoptosis-inducing factor and accompanied by formation of a DNA ladder (Lu \& Wolfe, 2001; Akematsu \& Endoh, 2010), as is characteristic of apoptosis in metazoans. Two genes encoding homologs of apoptosis-inducing factor are found in the Tetrahymena thermophila genome (Akematsu \& Endoh, 2010). Knock-out of an apoptosis-inducing factor gene gave ambiguous results (Akematsu et al., 2012); either knock-out was incomplete, only one gene out of several ohnologs was knocked-out (when not very similar), or the effect was compensated by a mechanism yet to be determined. Unexpectedly the nuclease involved is of mitochondrial origin, as found with Tetrahymena thermophila; this enzyme, TMN1 (Tetrahymena mitochondrial nuclease), also occurs in Paramecium tetraurelia (Osada etal., 2014). Probing Tetrahymena extracts with caspase substrates and caspase inhibitors was compatible with the presence of some form of caspase activity (Ejercito \& Wolfe, 2003; González et al., 2008). However, no gene for caspase proper has been found so far (Akematsu et al., 2012). Molecular identification and characterisation of caspase-like proteins, similar to the paracaspase of Dictyostelium discoideum or the metacaspase of Plasmodium falciparum and Arabidopsis thaliana (Uren etal., 2000), would be crucial.

In summary, some gross morphological traits and DNA ladder formation are in common for programmed cell death in metazoans and 'programmed nuclear death' in ciliates where, however, some essential features are either different or still require elucidation.

\section{GONGLUSIONS}

(1) Signalling implies long- and short-range information transfer, to achieve specific targeting and specific responses. Signalling regulates many aspects of subcellular dynamics. In principle, ciliates use similar, although not all, mechanisms for signalling and trafficking as metazoans, including $\mathrm{Ca}^{2+}$ and cyclic nucleotides, protein kinases and phosphatases, etc.

(2) Microtubules serve as long-range signals in different parts of the ciliate cell. Short-range signals include cues for molecular interactions, as is the case with SNARE proteins and small GTPases.

(3) Signalling by $\mathrm{Ca}^{2+}$ is elaborate in ciliates, including different influx and intracellular release channels. A local $\mathrm{Ca}^{2+}$ signal probably is required for vesicle docking and certainly for eventual vesicle/membrane fusion. The signal-transducing $\mathrm{Ca}^{2+}$ sensor remains unidentified in ciliates.

(4) Different, specifically localised influx channels regulate ciliary responses. Depolarisation via channels in the non-ciliary membrane activates voltage-dependent $\mathrm{Ca}^{2+}$ channels in the cilia and thereby ciliary beat reversal. Hyperpolarisation accelerates forward swimming.

(5) $\mathrm{Ca}^{2+}$, cyclic nucleotides and protein phosphorylation cooperate in the regulation of ciliary beating. Ser/Thr phosphorylation prevails over $\mathrm{Tyr}$ phosphorylation. In ciliates, dedicated Tyr-kinases probably do not contribute to signalling beyond a MAPK activity chain. Much work remains to be done.

(6) In ciliates, specific isoforms of CRCs related to $\mathrm{IP}_{3} \mathrm{Rs}$ and RyR-related $\mathrm{Ca}^{2+}$-release channels are localised to specific vesicles undergoing trafficking. This allows for very locally restricted signalling and mutually independent activation of widely different processes. Probably cADPR and NAADP are additional second messengers for activating CRCs in some $\mathrm{Ca}^{2+}$ stores of ciliates.

(7) Stimulated exocytosis is initiated by $\mathrm{Ca}^{2+}$ release from cortical stores (alveolar sacs), superimposed on store-operated $\mathrm{Ca}^{2+}$-entry, $\mathrm{SOC}(\mathrm{E})$. This is an old mechanism conserved in lineages up to man, e.g. in skeletal muscle. Molecules serving for coupling of the store with 
the outside medium are unknown and possibly different in ciliates.

(8) Important aspects still to be elaborated, or even to be detected in ciliates are trimeric GTP-binding proteins and associated receptors, GPCRs being important signal transducers in metazoans. The same is true of organelle-specific targeting and effectors, e.g. of molecular cues mediating organelle specificity of SNAREs and of small GTPases.

(9) The identity and distribution of mechanosensitive channels, established in the plasmalemma, but expected also for food vacuoles and the contractile vacuole complex, have to be settled on the basis of the molecular candidates available from ciliate databases. Also to be scrutinised are details concerning the mechanism of transmission of chemical information to mechanical work in cilia.

(10) Epigenetic signalling, since its discovery in fungi and ciliates, is a particularly rewarding field for future research.

(11) One can now compare the molecular background of signalling in representatives of the two main evolutionary lineages, Uni- and Bikonta (Plattner \& Verkhratsky, 2015). This includes choanoflagellates (Cai et al., 2015) and animals (Cai, 2008; Cai etal., 2015) on the one hand and ciliates (Plattner, 2015b) and plants (Edel \& Kudla, 2015) on the other. Such a comparison reveals the following interesting features.

(12) Some signalling elements have been maintained throughout eukaryotes, unikonts and bikonts. Among them are $\mathrm{Ca}^{2+}$, calmodulin, cyclic nucleotide-activated protein kinases, $\mathrm{H}^{+}$-ATPase/pump, Rab proteins and SNAREs.

(13) Some functions are specific to bikonts. For example, $\mathrm{Ca}^{2+} / \mathrm{CaM}$-activated protein kinase of unikonts is replaced by CDPK in bikonts (Sections III, IX). Also in ciliates 'synaptobrevins' are longins, as in plants (Section IV.2).

(14) Some other functions occurring in ciliates are found in unikonts, while they are reduced or abolished in plants. For instance, the function of calcineurin is greatly reduced in plants, with only the B-subunit being conserved. In plants it serves for the regulation of channel protein phosphorylation to defend against stress (Edel \& Kudla, 2015). By contrast, ciliates express both subunits, A and B, probably with pleiotropic functions (Sections XII.3 and XII.4). Dimeric calcineurin also occurs in unikonts, from myxamoebae to man. Both subunits are used by Dictyostelium discoideum for stress control (Thewes et al., 2014) and, in man, for immune responses as well as for signal transduction and information storage in the central nervous system. Voltage-dependent $\mathrm{Ca}^{2+}$-influx channels are absent from plants, as are $\mathrm{IP}_{3} \mathrm{R}$ and RyR-type GRCs. These are present in ciliates as well as in unikonts up to mammals (Plattner \& Verkhratsky, 2012, 2015).

(15) There are examples of the presence of some signalling pathways in animals and plants, but with fragmentary evidence for ciliates. There are only suggestions for the occurrence of trimeric G-proteins in ciliates, although they are well documented for unikonts, from Dictyostelium discoideum (Manahan etal., 2004) to man, and for 'higher' bikonts, such as plants (Ishida et al., 2014). One may expect their identification also in ciliates. No synaptotagmin has been found in ciliates, in contrast to animals (Section IV.3 and IX.1) and plants (Craxton, 2004). However, ciliates contain synaptotagmin-like proteins, comparable to the 'extended synaptotagmins' of some animal cells, which might serve as a $\mathrm{Ca}^{2+}$ sensor, e.g. during exocytosis.

(16) The use of Tyr phosphorylation for signalling is now attributed not only to metazoans but increasingly also to plants (De la Fuente van Bentem \& Hirt, 2008; Ghelis, 2011) - in contrast to ciliates, where it contributes to the MAPK signalling pathway (Section IX.2).

(17) In summary, in the signalling machinery of ciliates we observe traits in common to all eukaryotes, or in common with either other bikonts or unikonts. For some proteins common precursor molecules are likely to occur in protozoa, e.g. Ins $\mathrm{P}_{3}$ - and $\mathrm{RyRs}$ in ciliates (Plattner \& Verkhratsky, 2012, 2015), whose descendants may have further differentiated in unikonts and been lost in plants. A strict separation of the two lineages on the basis of signalling is not possible. Thus, with regard to signalling, ciliates appear to be at the crossroads between uni- and bikonts. Further investigations may contribute to the ongoing controversies about the validity of deep-rooting phylogeny.

(18) The golden thread running through signalling in ciliates is that crucial pathways appeared early in evolution and have been maintained, with variations on a basic theme, over a billion years.

\section{AGKNOWLEDGEMENTS}

The author was supported by the German Research Council (Deutsche Forschungsgemeinschaft).

\section{REFERENGES}

Adl, S. M., Simpson, A. G., Farmer, M. A., Andersen, R. A., Anderson, O. R., Barta, J. R., Bowser, S. S., Brugerolle, G., Fensome, R. A., FredericQ, S., James, T. Y., Karpov, S., Kugrens, P., Krug, J., Lane, C. E., Lewis, L. A., Lodge, J., Lynn, D. H., Mann, D. G., McCourt, R. M., Mendoza, L., Moestrup, O., Mozley-Standridge, S. E., Nerad, T. A., Shearer, G. A., Smirnov, A. V., Spiegel, F. W. \& Taylor, M. F. (2005). The new higher level classification of eukaryotes with emphasis on the taxonomy of protists. Fournal of Eukaryotic Microbiology 52, 399-451.

Adl, S. M., Simpson, A. G. B., Lane, C. E., Lukes, J., Bass, D., Bowser, S. S., Brown, M. W., Burki, F., Dunthorn, M., Hampl, V., Heiss, A., Hoppenrath, M., Lara, E., le Gall, L., Lynn, D. H., McManus, H., Mitchell, E. A. D., Mozley-Stanridge, S. E., Parfrey, L. W., Pawlowski, J., Rueckert, S., Shadwick, L., Schoch, C. L., Smirnov, A. \& Spiegel, F. W. (2012). The revised classification of eukaryotes. Fournal of Eukaryotic Microbiology 59, 429-493.

Adoutte, A., Delgado, P., Fleury, A., Levilliers, N., Lainé, M. G., Marty, M. C., Boisvieux-Ulrich, E. \& Sandoz, D. (1991). Microtubule diversity in ciliated cells: evidence for its generation by post-translational modification in the axonemes of Paramecium and quail oviduct cells. Biology of the Cell 71, 227-245.

Akematsu, T. \& Endoh, H. (2010). Role of apoptosis-inducing factor (AIF) in programmed nuclear death during conjugation in Tetrahymena thermophila. BMC Cell Biology 11, 13.

Akematsu, T., Kobayashi, T., Osada, E., Fukuda, Y., Endoh, H. \& Pearlman, R. E. (2012). Programmed nuclear death and its relation to apoptosis and autophagy during sexual reproduction in Tetrahymena thermophila. Fapanese Fournal of Protozoology $45,1-15$. 
Akematsu, T., Pearlman, R. E. \& Endoh, H. (2010). Gigantic macroautophagy in programmed nuclear death of Tetrahymena thermophila. Autophagy 6, 901-911.

Allen, R. D. (1974). Food vacuole membrane growth with microtubule-associated membrane transport in Paramecium. Fournal of Cell Biology 63, 904-922.

Allen, R. D. \& FoK, A. K. (2000). Membrane trafficking and processing in Paramecium. International Review of Cytology 198, 277-318.

Allen, R. D., MA, L. \& FoK, A. K. (1993). Acidosomes: recipients of multiple sources of membrane and cargo during development and maturation. Fournal of Cell Science 106, $411-422$.

Allen, R. D. \& Naitoh, Y. (2002). Osmoregulation and contractile vacuoles of protozoa. International Review of Cytology 215, 351-394.

Allen, P. D., Tominaga, T. \& Naitoh, Y. (2009). The contractile vacuole complex and cell volume control in protozoa. In Osmotic and Ionic Regulation: Cells and Animals (ed. D. H. Evans), pp. 69-106. CRC Press, Taylor and Francis Group, Boca Raton.

Anantharaman, V., Abhiman, S., de Souza, R. F. \& Aravind, L. (2011). Comparative genomics uncovers novel structural and functional features of the heterotrimeric GTPase signaling system. Gene 475, 63-78.

Anishinin, A. \& Kung, C. (2013). Stiffened lipid platforms at molecular force foci. Proceedings of the National Academy of Sciences of the United States of America 110, 4886-4892.

Anitei, M. \& Hoflack, B. (2012). Bridging membrane and cytoskeleton dynamics in the secretory and endocytic pathways. Nature Cell Biology 14, 11-19.

AnN, K. S. \& Nelson, D. L. (1995). Protein substrates for cGMP-dependent protein phosphorylation in cilia of wild type and atalanta mutants of Paramecium. Cell Motility and the Cytoskeleton 30, 252-260.

Antony, C. \& CAPDEville, Y. (1989). Uneven distribution of surface-antigens during antigenic variation in Paramecium primaurelia. Fournal of Cell Science 92, 205-215.

Arambasic, M., Sandoval, P. Y., Hoehener, C., Singh, A., Swart, E. C. \& Nowacki, M. (2014). Pdsg1 and Pdsg2, novel proteins involved in developmental genome remodelling in Paramecium. PLoS One 9, e112899.

Arnaiz, O., Goût, J. F., Bétermier, M., Bouhouche, K., Cohen, J., Duret, L., Kapusta, A., Meyer, E. \& Sperling, L. (2010). Gene expression in a paleopolyploid: a transcriptome resource for the ciliate Paramecium tetraurelia. BMC Genomics 11, 547.

Arslanyolu, M. (2007). Cloning and partial characterization of Tetrahymena thermophila mitogen activated protein kinase 3 (TtMPK3) gene. Fournal of Applied Biological Sciences $1,1-11$.

Artalejo, G. R., Henley, J. R., McNiven, M. A. \& Palfrey, H. G. (1995). Rapid endocytosis coupled to exocytosis in adrenal chromaffin cells involves $\mathrm{Ca}^{2+}$, GTP, and dynamin but not clathrin. Proceedings of the National Academy of Sciences of the United States of America 92, 8328-8332.

Arvan, P. \& Castle, D. (1998). Sorting and storage during secretory granule biogenesis: looking backward and looking forward. Biochemical fournal 332, 593-610.

Aubusson-Fleury, A., Bricheux, G., Damaj, R., Lemullois, M., Coffe, G., Donnadieu, F., Koll, F., Vigues, B. \& Bouchard, P. (2013). Epiplasmins and epiplasm in Paramecium: the building of a submembraneous cytoskeleton. Protist 164, $451-469$.

Aubusson-Fleury, A., Lemullois, M., de Loubresse, N. G., Laligne, G., Cohen, J., Rosnet, O., Jerka-Dziadosz, M., Beisson, J. \& Koll, F. (2012). The conserved centrosomal protein FOR20 is required for assembly of the transition zone and basal body docking at the cell surface. Fournal of Cell Science 125, 4395-4404.

Aufderheide, K. J. (1978). Motility events of trichocyst insertion in Paramecium tetraurelia. Fournal of Protozoology 25, 362-365

Aury, J.-M., Jaillon, O., Duret, L., Noel, B., Jubin, C., Porcel, B. M., Ségurens, B., Daubin, V., Anthouard, V., Aiach, N., Arnaiz, O., Billaut, A., Beisson, J., Blanc, I., Bouhouche, K., Camara, F., Duharcourt, S., Guigo, R., Godendeau, D., Katinka, M., Keller, A.-M., Kissmehl, R., Klotz, G., Koll, F., LeMouel, A., Lepère, G., Malinsky, S., Nowacki, M., Nowak, J., Plattner, H., Poulain, J., Ruiz, F., Serrano, V., Zagulski, M., Dessen, P., Bétermier, M., Weissenbach, J., Scarpelli, C., Schächter, V., Sperling, L., Meyer, E., Cohen, J. \& Wincker, P. (2006). Global trends of whole genome duplications revealed by the genome sequence of the ciliate Paramecium tetraurelia. Nature 444, 171-178.

Avraham, R. \& Yarden, Y. (2011). Feedback regulation of EGFR signalling: decision making by early and delayed loops. Nature Reviews Molecular Cell Biology 12, 104-117.

BaKsh, S. \& BURAKofF, S. J. (2000). The role of calcineurin in lymphocyte activation. Seminars in Immunology 12, 405-415.

BaLABAN, R. S. (2009). The role of $\mathrm{Ca}^{2+}$ signaling in the coordination of mitochondrial ATP production with cardiac work. Biochimica et Biophysica Acta 1787, 1334-1341.

Baldauf, S. L., Roger, A. J., Wenk-Siefert, I. \& Doolittlee, W. F. (2000). A kingdom-level phylogeny of eukaryotes based on combined protein data. Science 290, $972-977$.

Baranasic, D., Oppermann, T., Cheaib, M., Cullum, J., Schmidt, H. \& Simon, M. (2014). Genomic characterization of variable surface antigens reveals a telomer position effect as a prerequisite for RNA interference-mediated silencing in Paramecium tetraurelia. mBio 5(6), e01328-14.

Barbrook, A. C., Howe, C. J., Kurniawan, D. P. \& Tarr, S. J. (2010). Organization and expression of organellar genomes. Philosophical Transactions of the Royal Society of London. Series B: Biological Sciences 365, 785-797.
BARFORD, D. (2010). The structure and topology of protein serine/threonine phosphatases. In Handbook of Cell Signaling (Volume 2, eds R. A. Bradshaw and E. A. Dennis), pp. 677-681. Elsevier, New York.

BARRITT, G. J. (1999). Receptor-activated $\mathrm{Ca}^{2+}$ inflow in animal cells: a variety of pathways tailored to meet different intracellular $\mathrm{Ca}^{2+}$ signalling requirements. Biochemical fournal 337, 153-169.

Basten, S. G. \& Giles, R. H. (2013). Functional aspects of primary cilia in signaling, cell cycle and tumorigenesis. Cilia 2,6 .

Beale, G. H. \& Preer, J. R. JR. (2008). Paramecium Genetics and Epigenetics. CRC Press, Boca Raton.

Beisson, J. (2008). Preformed cell structure and cell heredity. Prion 2, 1-8.

Beisson, J. \& Jerka-Dziadosz, M. (1999). Polarities of the centriolar structure: morphogenetic consequences. Biology of the Cell 91, 367-378.

Beisson, J. \& Sonneborn, T. M. (1965). Cytoplasmic inheritance of the organization of the cell cortex in Paramecium aurelia. Proceedings of the National Academy of Science of the United States of America 53, 275-282.

Bell, W. E., Preston, R. R., Yano, J. \& Van Houten, J. L. (2007). Genetic dissection of attractant-induced conductances in Paramecium. Fournal of Experimental Biology 210, 357-365.

Bemm, F., Schwarz, R., Forster, F. \& Schultz, J. (2009). A kinome of 2600 in the ciliate Paramecium tetraurelia. Federation of European Biological Societies Letters $\mathbf{5 8 3}$ $3589-3592$.

Benwakrim, A., Tremoliere, A., Labarre, J. \& Capdeville, Y. (1998). The lipid moiety of the GPI-anchor of the major plasma membrane proteins in Paramecium primaurelia is a ceramide: variation of the amide-linked fatty acid composition as a function of growth temperature. Protist 149, 39-50.

Berridge, M. J. (2006). Calcium microdomains: organization and function. Cell Calcium 40, 405-412.

Berridge, M. J., Bootman, M. D. \& Lipp, P. (1998). Calcium - a life and death signal. Nature $395,645-648$.

Berridge, M. J., Bootman, M. D. \& Roderick, H. L. (2003). Calcium signalling: dynamics, homeostasis and remodelling. Nature Reviewes Molecular Cell Biology 4, $517-529$.

Beyenbach, K. W. \& Wieczorek, H. (2006). The V-type H ${ }^{+}$ATPase: molecular structure and function, physiological roles and regulation. Fournal of Experimental Biology 209, 577-589.

Bezprozvanny, I. (2005). The inositol 1,4,5-trisphosphate receptor. Cell Calcium 38 , 261-272.

Bick, A. G., Calvo, S. E. \& Moотha, V. K. (2012). Evolutionary diversity of the mitochondrial calcium uniporter. Science 336, 886.

Bilinski, M., Plattner, H. \& Matt, H. (1981). Secretory protein decondensation as a distinct, $\mathrm{Ca}^{2+}$-mediated event during the final steps of exocytosis in Paramecium cells. Fournal of Cell Biology 88, 179-188.

BLATT, M. R. (2000). Cellular signaling and volume control in stomatal movements in plants. Annual Review of Cell and Developmental Biology 16, 221-241.

BLoodgood, R. A. (2012). The future of ciliary and flagellar membrane research. Molecular Biology of the Cell 23, 2407-2411.

Blum, J. J., Hayes, A., Jamieson, G. A. \& Vanaman, T. C. (1980). Calmodulin confers calcium sensitivity on ciliary dynein ATPase. Fournal of Cell Biology 87, $386-397$.

Bockaert, J. \& Pin, J. P. (1999). Molecular tinkering of G protein-coupled receptors: an evolutionary success. European Molecular Biology Organisation fournal 18, 1723-1729.

Bonifacino, J. S. (2014). Adaptor proteins involved in polarized sorting. Fournal of Cell Biology 204, 7-17.

Bonini, N. M., Gustin, M. C. \& Nelson, D. L. (1986). Regulation of ciliary motility by membrane potential in Paramecium: a role for cyclic AMP. Cell Motility and the Cytoskeleton 6, 256-272.

Bonini, N. M. \& Nelson, D. L. (1990). Phosphoproteins associated with cyclic nucleotide stimulation of ciliary motility in Paramecium. Fournal of Cell Science 95, 219-230.

Bowman, G. R., Eide, N. C., Morgan, G., Winey, M \& Turkewitz, A. P. (2005). Core formation and the acquisition of fusion competence are linked during secretory granule maturation in Tetrahymena. Traffic 6, 303-323.

Bradford, W., Buckholz, A., Morton, J., Price, C., Jones, A. M. \& Urano, D. (2013). Eukaryotic G protein signaling evolved to require G protein-coupled receptors for activation. Science Signaling 6 , ra37.

BRÄUcker, R., MACHEMER-RöHnisch, S. \& MACHEMER, H. (1994). Graviresponse in Paramecium caudatum and Didinium nasutum examined under varied hypergravity conditions. Fournal of Experimental Biology 197, 271-294.

Brehm, P. \& EcKert, R. (1978). Calcium entry leads to inactivation of calcium channel in Paramecium. Science 202, 1203-1206.

Bright, L. J., Kambesis, N., Nelson, S. B., Jeong, B. \& Turkewitz, A. P. (2010). Comprehensive analysis reveals dynamic and evolutionary plasticity of Rab GTPases and membrane traffic in Tetrahymena thermophila. PLoS Genetics 6, e1001155.

Briguglio, J. S., Kumar, S. \& Turkewitz, A. P. (2013). Lysosomal sorting receptors are essential for secretory granule biogenesis in Tetrahymena. Fournal of Cell Biology 203, 537-550. 
Brown, D., Paunescu, T. G., Breton, S. \& Marshansky, V. (2009). Regulation of the V-ATPase in kidney epithelial cells: dual role in acid-base homeostasis and vesicle trafficking. Fournal of Experimental Biology 212, 1762-1772.

Brunk, C. F., Lee, L. G., Tran, A. B. \& Li, J. (2003). Complete sequence of the mitochondrial genome of Tetrahymena thermophila and comparative methods for identifying highly divergent genes. Nucleic Acids Research 31, 1673-1682.

Buhse, H. E. Jr. (1967). Microstome-macrostome transformation in Tetrahymena vorax strain V2 type $\mathrm{S}$ induced by a transforming principle, stomatin. Foumal of Protozoology 14, 608-613.

Buonanno, F., Anesi, A., Guella, G., Kumar, S., Bharti, D., La Terza, A., Quassinti, L., Bramucci, M. \& Ortenzi, C. (2014). Chemical offense by means of toxicysts in the freshwater ciliate, Coleps hirtus. Fournal of Eukaryotic Microbiology 61, 293-304.

Buonanno, F., Harumoto, T. \& Ortenzi, C. (2013). The defensive function of trichocysts in Paramecium tetraurelia against metazoan predators compared with the chemical defense of two species of toxin-containing ciliates. Zoological Science 30, $255-261$.

Burnstock, G. \& Verkhratsky, A. (2009). Evolutionary origins of the purinergic signalling system. Acta Physiologica (Oxford) 195, 415-447.

CAI, X. (2008). Unicellular $\mathrm{Ca}^{2+}$ signaling 'toolkit' at the origin of metazoa. Molecular Biology and Evolution 25, 1357-1361.

Cai, X., Wang, X., Patel, S. \& Clapham, D. E. (2015). Insights into the early evolution of animal calcium signaling machinery: a unicellular point of view. Cell Calcium 57, 166-173.

Capdeville, Y. (2000). Paramecium GPI proteins: variability of expression and localization. Protist 151, 161-169.

Cavalier-Smith, T. (2010). Deep phylogeny, ancestral groups and the four ages of life. Philosophical Transactions of the Royal Society of London. Series B: Biological Sciences 365, $111-132$.

Cervia, D., Catalani, E., Belardinelli, M. C., Perrotta, C., Picchietti, S, Alimenti, C., Casini, G., Fausto, A. M. \& Vallesi, A. (2013). The protein pheromone Er-1 of the ciliate Euplotes raikovi stimulates human T-cell activity: involvement of interleukin-2 system. Experimental Cell Research 319, 56-67.

Chalker, D. L., Meyer, E. \& Mochizuki, K. (2013). Epigenetics of ciliates. Cold Spring Harbor Perspectives in Biology 5, a017764.

Chalker, D. L. \& YaO, M. C. (2011). DNA elimination in ciliates: transposon domestication and genome surveillance. Annual Review of Genetics 45, 227-246.

Chen, J., Gao, X., Wang, B., Chen, F., Wu, N. \& Zhang, Y. (2014). Proteomic approach to reveal the proteins associated with encystment of the ciliate Euplotes encysticus. PLoS One $\mathbf{9}$, e97362.

Chen, Z. Y., Ieraci, A., Teng, H., Dall, H., Meng, C. X., Herrera, D. G. Nykjaer, A., Hempstead, B. L. \& Lee, F. S. (2005). Sortilin controls intracellular sorting of brain-derived neurotrophic factor to the regulated secretory pathway. Journal of Neuroscience 25, 6156-6166.

Chien, Y. H., Werner, M. E., Stubbs, J., Joens, M. S., Li, J., Chien, S. Fitzpatrick, J. A., Mitchell, B. J. \& Kintner, C. (2013). Bbofl is required to maintain cilia orientation. Development 140, 3468-3477.

Chilcoat, N. D. \& Turkewitz, A. P. (1997). In vivo analysis of the major exocytosis-sensitive phosphoprotein in Tetrahymena. Fournal of Cell Biology 139, $1197-1207$.

Choi, J., Tanaka, K., Cao, Y., Qi, Y., Qiu, J., Liang, Y., Lee, S. Y. \& Stacey, G. (2014). Identification of a plant receptor for extracellular ATP. Science 343, 290-294.

Choksi, S. P., Lauter, G., Swoboda, P. \& Roy, S. (2014). Switching on cilia: transcriptional networks regulating ciliogenesis. Development 141, 1427-1441.

Christensen, S. T., Guerra, C. F., Awan, A., Wheatley, D. N. \& Satir, P. (2003). Insulin receptor-like proteins in Tetrahymena thermophila ciliary membranes. Current Biology 13, R50-R52.

Christensen, K. A., Myers, J. T. \& Swanson, J. A. (2002).pH-dependent regulation of lysosomal calcium in macrophages. Fournal of Cell Science 115, 599-607.

Churamani, D., Hooper, R., Rahman, T., Brailoiu, E. \& Patel, S. (2013). The $\mathrm{N}$-terminal region of two-pore channel 1 regulates trafficking and activation by NAADP. Biochemical fournal 453, 147-151.

Cipriano, D. J., Jung, J., Vivona, S., Fenn, T. D., Brunger, A. T. \& Bryant, Z. (2013). Processive ATP-driven substrate disassembly by the N-ethylmaleimide-sensitive factor (NSF) molecular machine. Fournal of Biological Chemistry 288, 23436-23445.

Clapham, D. E. (2007). Calcium signaling. Cell 131, 1047-1058.

Clark, T. G. \& Forney, J. D. (2003). Free-living and parasitic ciliates. In Antigenic Variation (eds A. Craig and R. Scherf), pp. 375-402. Elsevier, London.

Clark, K. D., Hennessey, T. M. \& Nelson, D. L. (1993). External GTP alters the motility and elicits an oscillating membrane depolarization in Paramecium tetraurelia. Proceedings of the National Academy of Sciences of the United States of America 90, 3782-3786.

Condou, C., Yan, Z., Obsil, T., Huidobro-Toro, J. P. \& Stojilkovic, S. S. (2011). Activation and regulation of purinergic P2X receptor channels. Pharmacological Reviewes 63, 641-683.

Cogoli, A., Tschopp, A. \& Fuchs-Bislin, P. (1984). Circumnutation observed without a significant gravitational force in spaceflight. Science 225, 229-231.
Cole, E. S., Cassidy-Hanley, D., Pinello, J. F., Zeng, H., Hsueh, M., Kolbin, D., Ozzello, C., Giddings, T., Winey, M. \& Clark, T. G. (2014). Function of the male-gamete-specific fusion protein HAP2 in a seven-sexed ciliate. Current Biology 24, 2168-2173.

Coluccio, L. M. E. (2008). Myosin. A Superfamily of Molecular Motors. Springer, Dordrecht.

Coria, A. S., Masseroni, M. L. \& Diaz Anel, A. M. (2014). Regulation of PKD1-mediated Golgi to cell surface trafficking by G $\alpha$ q subunits. Biology of the Cell 106, 30-43.

Coste, B., Mathur, J., Schmidt, M., Earley, T. J., Ranade, S., Petrus, M. J., Dubin, A. E. \& Patapoutian, A. (2010). Piezol and Piezo2 are essential components of distinct mechanically activated cation channels. Science 330, 55-60.

Craxton, M. (2004). Synaptotagmin gene content of the sequenced genomes. BMC Genomics 5, 43 (doi: 10.1186/1471-2164-5-43).

DACKs, J. B. \& Field, M. C. (2007). Evolution of the eukaryotic membrane-trafficking system: origin, tempo and mode. Fournal of Cell Science 120, 2977-2985.

Dalghi, M. G., Fernandez, M. M., Ferreira-Gomes, M., Mangialavori, I. C., Malchiodi, E. L., Strehler, E. E. \& Rossi, J. P. (2013). Plasma membrane calcium ATPase activity is regulated by actin oligomers through direct interaction. Fournal of Biological Chemistry 288, 23380-23393.

De Franceschi, N., Wild, K., Schlacht, A., Dacks, J. B., Sinning, I. \& Filippini, F. (2014). Longin and GAF domains: structural evolution and adaptation to the subcellular trafficking machinery. Traffic 15, 104-121.

De la Fuente van Bentem, S. \& Hirt, H. (2008). Protein tyrosine phosphorylation in plants: more abundant than expected? Trends in Plant Science 14, 71-76.

Dentler, W. L. (1988). Fractionation of Tetrahymena ciliary membranes with Triton S-114 and the identification of a ciliary membrane ATPase. Fournal of Cell Biology 107, 2679-2688.

Denton, R. M. (2009). Regulation of mitochondrial dehydrogenases by calcium ions. Biochimica et Biophysica Acta 1787, 1309-1316.

De Ondarza, J., Symington, S. B., Van Houten, J. L. \& Clark, J. M. (2003). G-protein modulators alter the swimming behavior and calcium influx of Paramecium tetraurelia. Fournal of Eukaryotic Microbiology 50, 349-355.

Dorrell, R. G., Butterfield, E. R., Nisbet, R. E. R. \& Howe, C. J. (2013). Evolution: unveiling early aveolates. Current Biology 23, R1093-R1096.

Duharcourt, S., Lepère, G. \& Meyer, E. (2009). Developmental genome rearrangements in ciliates: a natural genomic subtraction mediated by non-coding transcripts. Trends in Genetics 25, 344-350.

Duret, L., Cohen, J., Jubin, C., Dessen, P., Goût, J.-F., Mousset, S., Aury, J.-M., Jaillon, O., Noël, B., Arnaiz, O., Bétermier, M., Wincker, P. \& Meyer, E. (2008). Analysis of the sequence variability in the macronuclear DNA of Paramecium tetraurelia. Genome Research 18, 585-596.

Eckert, R. \& Brehm, P. (1979). Ionic mechanisms of excitation in Paramecium. Annual Review of Biophysics and Bioengineering 8, 353-383.

Edel, K. H. \& Kudla, J. (2015). Increasing complexity and versatility: how the calcium signaling toolkit was shaped during plant land colonization. Cell Calcium 57, 231-246.

Eisen, J. A., Coyne, R. S., Wu, M., Wu, D., Thiagarajan, M., Wortman, J. R., Badger, J. H., Ren, Q, Amedeo, P., Jones, K. M., Tallon, L. J., Delcher, A. L., Salzberg, S. L., Silva, J. C., Haas, B. J., Majoros, W. H., Farzad, M., Carlton, J. M., Smith, R. K. Jr., Garg, J., Pearlman, R. E., Karrer, K. M. Sun, L., Manning, G., Elde, N. C., Turkewitz, A. P., Asai, D. J., Wilkes, D. E., Wang, Y., Cai, H., Collins, K., Stewart, B. A., Lee, S. R., Wilamowska, K., Weinberg, Z., Ruzzo, W. L., Wloga, D., Gaertig, J., Frankel, J., Tsao, C. C., Gorovsky, M. A., Keeling, P. J., Waller, R. F., Patron, N. J., Cherry, J. M., Stover, N. A., Krieger, C. J., del Toro, C., Ryder, H. F., Williamson, S. C., Barbeau, R. A., Hamilton, E. P. \& Orias, E. (2006). Macronuclear genome sequence of the ciliate Tetrahymena thermophila, a model eukaryote. PLoS Biology 4 , e286.

Ejercito, M. \& Wolfe, J. (2003). Caspase-like activity is required for programmed nuclear elimination during conjugation in Tetrahymena. Fournal of Eukaryotic Microbiology 50, 427-429.

Elde, N. G., Morgan, G., Winey, M., Sperling, L. \& Turkewitz, A. P. (2005). Elucidation of clathrin-mediated endocytosis in Tetrahymena reveals an evolutionarily convergent recruitment of dynamin. PLoS Genetics 1, e52.

El-Haddad, H., Przyborski, J. M., Kraft, L. G., McFadden, G. I., Waller, R. F. \& Gould, S. B. (2013). Characterization of TtALV2, an essential charged repeat motif protein of the Tetrahymena thermophila membrane skeleton. Eukaryotic Cell $\mathbf{1 2}$ 932-940.

Elwess, N. L. \& VAN Houten, J. L. (1997). Cloning and molecular analysis of the plasma membrane $\mathrm{Ca}^{2+}$-ATPase gene in Paramecium tetraurelia. Fournal of Eukaryotic Microbiology 44, 250-257.

Endoh, H. \& Kobayashi, T. (2006). Death harmony played by nucleus and mitochondria. Nuclear apoptosis during conjugation of Tetrahymena. Autophagy 2, $129-131$.

Ernst, I. M., Fliegert, R. \& Guse, A. H. (2013). Adenine dinucleotide second messengers and T-lymphocyte calcium signaling. Frontiers in Immunology 4, 259. 
Erxleben, C., Klauke, N., Flötenmeyer, M., Blanchard, M. P., Braun, C. \& Plattner, H. (1997). Microdomain $\mathrm{Ca}^{2+}$ activation during exocytosis in Paramecium cells. Superposition of local subplasmalemmal calcium store activation by local $\mathrm{Ca}^{2+}$ influx. Fournal of Cell Biology 136, 597-607.

Escoll, P., Rolando, M., Gomez-Valero, L. \& Buchrieser, G. (2013). From amoeba to macrophages: exploring the molecular mechanisms of Legionella pneumophila infection in both hosts. Current Topics in Microbiology and Immunology 376, $1-34$

Esseltine, J. L. \& Scott, J. D. (2013). AKAP signaling complexes: pointing towards the next generation of therapeutic targets? Trends in Pharmacological Sciences 34, 648-655.

Evans, T. C. \& Nelson, D. L. (1989). The cilia of Paramecium tetraurelia contain both $\mathrm{Ca}^{2+}$-dependent and $\mathrm{Ca}^{2+}$-inhibitable calmodulin-binding proteins. Biochemical fournal 259, 385-396.

Farhan, H., Wendeler, M. W., Mitrovic, S., Fava, E., Silberberg, Y., Sharan, R., Zerial, M. \& Hauri, H. P. (2010). MAPK signaling to the early secretory pathway revealed by kinase/phosphatase functional screening. Fournal of Cell Biology 189, 997-1011.

Farrell, A., Thirugnanam, S., Lorestani, A., Dvorin, J. D., Eidell, K. P., Ferguson, D. J., Anderson-White, B. R., Duraisingh, M. T., Marth, G. T. \& Gubbels, M. J. (2012). A DOC2 protein identified by mutational profiling is essential for apicomplexan parasite exocytosis. Science 335, 218-221.

Fasshauer, D., Sutton, R. B., Brunger, A. T. \& Jahn, R. (1998). Conserved structural features of the synaptic fusion complex: SNARE proteins reclassified as Q- and R-SNAREs. Proceedings of the National Academy of Sciences of the United States of America 95, 15781-15786.

FENCHEL, T. (2014). Respiration in heterotrophic unicellular eukaryotic organisms. Protist 165, 485-492.

Fielding, A. B., Schonteich, E., Matheson, J., Wilson, G., Yu, X., Hickson, G. R. X., Srivastava, S., Baldwin, S. A., Prekeris, R. \& Gould, G. W. (2005). Rab11-FIP3 and FIP4 interact with Arf6 and the exocyst to control membrane traffic in cytokinesis. European Molecular Biology Organisation fournal 24, 3389-3399.

Findeisen, F., Rumpf, C. H. \& Minor, D. L. Jr. (2013). Apo states of calmodulin and $\mathrm{CaBP} 1$ control $\mathrm{CaV} 1$ voltage-gated calcium channel function through direct competition for the IQ domain. Fournal of Molecular Biology 425, 3217-3234.

Flannagan, R. S., Jaumouille, V. \& Grinstein, S. (2012). The cell biology of phagocytosis. Annual Review of Pathology 7, 61-98.

Flötenmeyer, M., MomayeZi, M. \& Plattner, H. (1999). Immunolabeling analysis of biosynthetic and degradative pathways of cell surface components (glycocalyx) in Paramecium cells. European Fournal of Cell Biology 78, 67-77.

FoK, A. K. \& Allen, P. D. (1993). Membrane flow in the digestive cycle of Paramecium. In Membrane Traffic in Protozoa (ed. H. Plattner), pp. 311-337. JAI Press, Greenwich, London.

Fok, A. K., Yamauchi, K., Ishihara, A., Aihara, M. S., Ishida, M. \& Allen, R. D. (2002). The vacuolar-atpase of Paramecium multimicronucleatum: gene structure of the $\mathrm{B}$ subunit and the dynamics of the V-ATPase-rich osmoregulatory membranes. foumal of Eukaryotic Microbiology 49, 185-196.

ForGAC, M. (2007). Vacuolar ATPases: rotary proton pumps in physiology and pathophysiology. Nature Reviews Molecular Cell Biology 8, 917-929.

Fountain, S. J., Parkinson, K., Young, M. T., Cao, L., Thompson, C. R. \& NoRTH, R. A. (2007). An intracellular P2X receptor required for osmoregulation in Dictyostelium discoideum. Nature 448, 200-203.

Fraga, D., Sehring, I. M., Kissmehl, R., Reiss, M., Gaines, R., Hinrichsen, R. \& Plattner, H. (2010). Protein phosphatase 2B (PP2B, calcineurin) in Paramecium: partial characterization reveals that two members of the unusually large catalytic subunit family have distinct roles in calcium-dependent processes. Eukaryotic Cell 9, 1049-1063.

Frankel, J. (1973). Dimensions of control of cortical patterns in Euplotes - Role of preexisting structure, clonal life-cycle, and genotype. Fournal of Experimental Zoology 183, $71-94$.

Froissard, M., Keller, A. M., Dedieu, J. C. \& Cohen, J. (2004). Novel secretory vesicle proteins essential for membrane fusion display extracellular-matrix domains. Traffic 5, 493-502.

Fu, L., Miseta, A., Hunton, D., Marchase, R. B. \& Bedwell, D. M. (2000). Los of the major isoform of phosphoglucomutase results in altered calcium homeostasis in Saccharomyces cerevisiae. Fournal of Biological Chemistry 275, 5431-5440.

Fu, Z., Schroeder, M. J., Shabanowitz, J., Kaldis, P., Togawa, K., Rustgi, A. K., Hunt, D. F. \& Sturgill, T. W. (2005). Activation of a nuclear Cdc2-related kinase within a mitogen-activated protein kinase-like TDY motif by autophosphorylation and cyclin-dependent protein kinase-activating kinase. Molecular Cell. Biology 25, 6047-6064.

Fujishima, M. \& Kodama, Y. (2012). Endosymbionts in Paramecium. European fournal of Protistology 48, 124-137.

Galati, D. F., Bonney, S., Kronenberg, Z., Clarissa, C., Yandell, M., Elde, N. C., Jerka-Dziadosz, M., Giddings, T. H., Frankel, J. \& Pearson, G. G. (2014). DisAp-dependent striated fiber elongation is required to organize ciliary arrays. The fournal of Cell Biology 207, 705-715.
Galione, A., Evans, A. M., Ma, J., Parrington, J., Arredouani, A., Cheng, X. \& Zhu, M. X. (2009). The acid test: the discovery of two-pore channels (TPCs) as NAADP-gated endolysosomal $\mathrm{Ca}^{2+}$ release channels. Pflugers Archiv European Fournal of Physiology 458, 869-876.

Garcés, J. A., Hoey, J. G. \& Gavin, R. H. (1995). Putative myosin heavy and light chains in Tetrahymena: co-localization to the basal body-cage complex and association of the heavy chain with skeletal muscle actin filaments in vitro. Fournal of Cell Science 108, 869-881.

Garreau de Loubresse, N. (1993). Early steps of the secretory pathway in Paramecium: ultrastructural, immunochemical, and genetic analysis of trichocyst biogenesis. In Membrane Traffic in Protozoa (ed. H. Plattner), pp. 27-59. JAI Press, Greenwich, London.

Garreau de Loubresse, N., Gautier, M. G. \& Sperling, L. (1994). Immature secretory granules are not acidic in Paramecium: implications for sorting to the regulated pathway. Biology of the Cell 82, 139-147.

Gebauer, M., Watzke, D. \& Machemer, H. (1999). The gravikinetic response of Paramecium is based on orientation-dependent mechanotransduction. Naturwissenschaften 86, 352-356.

Gerashchenko, B. I. (2010). Proliferation of green Paramecium bursaria: a vision through flow cytometry. Cytometry. Part A 77, 111-112.

Geron, E., Schejter, E. D. \& Shilo, B. Z. (2013). Directing exocrine secretory vesicles to the apical membrane by actin cables generated by the formin mDial. Proceedings of the National Academy of Sciences of the United States of America 110, $10652-10657$.

GHELIS, T. (2011). Signal processing by protein tyrosine phosphorylation in plants. Plant Signaling and Behavior 6, 942-951.

Gillespie, P. G. \& Walker, R. G. (2001). Molecular basis of mechanosensory transduction. Nature 413, 194-202.

Gilley, D., Rudman, B. M., Preer, J. R. Jr. \& Polisky, B. (1990). Multilevel regulation of surface antigen gene expression in Paramecium tetraurelia. Molecular Cell. Biology 10, 1538-1544.

Gilligan, D. M. \& Satir, B. H. (1982). Protein phosphorylation/dephosphorylation and stimulus-secretion coupling in wild type and mutant Paramecium. Fournal of Biological Chemistry 257, 13903-13906.

Giordano, F., Saheki, Y., Idevall-Hagren, O., Colombo, S. F., Pirruccello, M., Milosevic, I, Gracheva, E O, Bagriantsey, S, N , Borgese, N \& De Camilli, P. (2013). PI(4,5) $\mathrm{P}_{2}$-dependent and $\mathrm{Ca}^{2+}$-regulated ER-PM interactions mediated by the extended synaptotagmins. Cell 153, 1494-1509.

Glancy, B., Willis, W. T., Chess, D. J. \& Balaban, R. S. (2013). Effect of calcium on the oxidative phosphorylation cascade in skeletal muscle mitochondria Biochemistry 52, 2793-2809.

Godlee, C. \& Kaksonen, M. (2013). Review series: from uncertain beginnings: initiation mechanisms of clathrin-mediated endocytosis. Fournal of Cell Biology 203, $717-725$.

Goldberg, J. M., Manning, G., Liu, A., Fey, P., Pilcher, K. E., Xu, Y. \& Smith, J. L. (2006). The Dictyostelium kinome--analysis of the protein kinases from a simple model organism. PLoS Genetics 2, e38.

GolinsKa, K. (1982). Regulation of ciliary pattern in Dileptus (Ciliata). 1. Sensory cilia and their conversion into locomotor cilia. Fournal of Embryology and Experimental Morphology 68, 99-114.

Gomperts, B. D., Kramer, I. M. \& Tatham, P. E. R. (2009). Signal Transduction. Second Edition 0. Elsevier, London, New York

Gonda, K., Komatsu, M. \& Numata, O. (2000). Calmodulin and $\mathrm{Ca}^{2+} /$ calmodulin-binding proteins are involved in Tetrahymena thermophile phagocytosis. Cell Structure and Function 25, 243-251.

Gonda, K., OAmi, K. \& TAKahashi, M. (2007). Centrin controls the activity of the ciliary reversal-coupled voltage-gated $\mathrm{Ca}^{2+}$ channels $\mathrm{Ca}^{2+}$-dependently. Biochemical and Biophysical Research Communications 362, 170-176.

González, A. M., Díaz, S., Gallego, A. \& Gutiérrez, J. C. (2008). Programmed nuclear death and other apoptotic-like phenomena in ciliated protozoa. In Programmed Cell Death in Protozoa (ed. J. M. Pérez Martín), pp. 143-159. Landes Bioscience and Springer Bioscience + Business Media, Georgetown.

Good, M. C., Zalatan, J. G. \& Lim, W. A. (2011). Scaffold proteins: hubs for controlling the flow of cellular information. Science 332, 680-686.

Goyette, G., Boulais, J., Carruthers, N. J., Landry, C. R., Jutras, I., Duclos, S., Dermine, J. F., Michnick, S. W., LaBoissiere, S., Lajoie, G., Barreiro, L., Thibault, P. \& Desjardins, M. (2012). Proteomic characterization of phagosomal membrane microdomains during phagolysosome biogenesis and evolution. Molecular and Cellular Proteomics 11, 1365-1377.

Gray, E. G. (1978). Synaptic vesicles and microtubules in frog motor endplates. Proceedings of the Royal Society of London B: Biological Sciences 203, 219-227.

Griffiths, G. M., Tsun, A. \& Stinchcombe, J. C. (2010). The immunological synapse: a focal point for endocytosis and exocytosis. Fournal of Cell Biology 189, $399-406$.

Grønlien, H. K., Stock, C., Aimara, M. S., Allen, R. D. \& Naitoh, Y. (2002). Relationship between the membrane potential of the contractile vacuole complex and its osmoregulatory activity in Paramecium multimicronucleatum. Fournal of Experimental Biology 205, 3261-3270. 
Grosshans, B. L., Ortiz, D. \& Novick, P. (2006). Rabs and their effectors: achieving specificity in membrane traffic. Proceedings of the National Academy of Sciences of the United States of America 103, 11821-11827.

Grothe, K., Hanke, C., Momayezi, M., Kissmehl, R., Plattner, H. \& Schultz, J. E. (1998). Functional characterization and localization of protein phosphatase type 2C from Paramecium. Fournal of Biological Chemistry 273, 19167-19172.

Gueron, S. \& Levit-Gurevich, K. (1999). Energetic considerations of ciliary beating and the advantage of metachronal coordination. Proceedings of the National Academy of Sciences of the United States of America 96, 12240-12245.

GuSE, A. H. (2012). Linking NAADP to ion channel activity: a unifying hypothesis. Science Signaling 5, pe18.

Haacke-bell, B. \& Plattner, H. (1987). Secretory lectins contained in trichocyst tips of Paramecium. European Fournal of Cell Biology 44, 1-9.

Haddad, A. \& Turkewitz, A. P. (1997). Analysis of exocytosis mutants indicates close coupling between regulated secretion and transcription activation in Tetrahymena. Proceedings of the National Academy of Sciences of the United States of America 94, $10675-10680$

Hamada, K. \& Miкoshiba, K. (2012). Revisiting channel allostery: a coherent mechanism in $\mathrm{IP}_{3}$ and ryanodine receptors. Science Signaling $\mathbf{5}$, pe24.

Hamasaki, T., Barkalow, K., Richmond, J. \& Satir, P. (1991). cAMP-stimulated phosphorylation of an axonemal polypeptide that copurifies with the $22 \mathrm{~S}$ dynein arm regulates microtubule translocation velocity and swimming speed in Paramecium. Proceedings of the National Academy of Sciences of the United States of America 88, 7918-7922.

Hamill, O. P. \& Martinac, B. (2001). Molecular basis of mechanotransduction in living cells. Physiological Revieres 81, 685-740.

Hardt, M. \& Plattner, H. (2000). Sub-second quenched-flow/X-ray microanalysis shows rapid $\mathrm{Ca}^{2+}$ mobilization from cortical stores paralleled by $\mathrm{Ca}^{2+}$ influx during synchronous exocytosis in Paramecium cells. European fournal of Cell Biology 79 $642-652$.

Harumoto, T. (1994). The role of trichocyst discharge and backward swimming in escaping behavior of Paramecium from Dileptus margaritifer. Fournal of Eukaryotic Microbiology 41, 560-564

Harumoto, T. \& Miyake, A. (1991). Defensive function of trichocysts in Paramecium. Foumal of Experimental Zoology 260, 84-92.

Harumoto, T. \& Miyake, A. (1993). Possible participation of surface antigens of Paramecium in predator-prey interaction. Fournal of Eukaryotic Microbiology 40, 27A.

Hauser, K., Kissmehl, R., Linder, J., Schultz, J. E., Lottspeich, F. \& Plattiner, H. (1997). Identification of isoforms of the exocytosis-sensitive phosphoprotein PP63/parafusin in Paramecium tetraurelia and demonstration of phosphoglucomutase activity. Biochemical foumal 323, 289-296.

Hauser, K., Pavlovic, N., Klauke, N., Geissinger, D. \& Plattner, H. (2000). Green fluorescent protein-tagged sarco(endo)plasmic reticulum $\mathrm{Ca}^{2+}$-ATPase overexpression in Paramecium cells: isoforms, subcellular localization, biogenesis of cortical calcium stores and functional aspects. Molecular Microbiology 37, 773-787.

Hausmann, K. (1978). Extrusive organelles in protists. International Review of Cytology $52,197-276$

HAY, J. C. (2007). Calcium: a fundamental regulator of intracellular membrane fusion? European Molecular Biology Organisation Reports 8, 236-240.

He, D., Fiz-Palacios, O., Fu, C. J., Fehling, J., Tsai, C. C. \& Baldauf, S. L. (2014). An alternative root for the eukaryote tree of life. Current Biology 24 $465-470$.

Heath, R. J. \& InSALL, R. H. (2008). Dictyostelium MEGAPs: F-BAR domain proteins that regulate motility and membrane tubulation in contractile vacuoles. Fournal of Cell Science 121, 1054-1064.

HeMmersbaCh, R. \& BRÄUCKER, R. (2002). Gravity-related behaviour in ciliates and flagellates. Advances in Space Biology and Medicine 8, 59-75.

Hemmersbach, R. \& BraUn, M. (2006). Gravity-sensing and gravity-related signaling pathways in unicellular model systems of protists and plants. Signal Transduction $\mathbf{6}$ $432-442$.

Hemmersbach, R., Voormanns, R., Bromeis, B., Schmidt, N., Rabien, H. \& Ivanova, K. (1998). Comparative studies of the graviresponses of Paramecium and Loxodes. Advances in Space Research 21, 1285-1289.

Hemmersbach, R., Wilczek, M., Stieber, C., Bräucker, R. \& Ivanova, K. (2002). Variable acceleration influences cyclic AMP levels in Paramecium biaurelia. Fournal of Gravitational Physiology 9, P267-268.

Hennessey, T. M. (2005). Responses of the ciliates Tetrahymena and Paramecium to external ATP and GTP. Purinergic Signalling 1, 101-110.

Hergovich, A. (2011). MOB control: reviewing a conserved family of kinase regulators. Cellular Signalling 23, 1433-1440.

Hinrichsen, R. D. \& Blackshear, P. J. (1993). Regulation of peptide-calmodulin complexes by protein kinase $\mathrm{C}$ in vivo. Proceedings of the National Academy of Sciences of the United States of America 90, 1585-1589.

Hinshaw, J. E. (2000). Dynamin and its role in membrane fission. Annual Review of Cell and Developmental Biology 16, 483-519.

Hochstrasser, M. \& Nelson, D. L. (1989). Cyclic AMP-dependent protein kinase in Paramecium tetraurelia. Its purification and the production of monoclonal antibodies against both subunits. Fournal of Biological Chemistry 264, 14510-14518.
Höhne-Zell, B., Knoll, G., Riedel-Gras, U., Hofer, W. \& Plattner, H. (1992). A cortical phosphoprotein ('PP63') sensitive to exocytosis triggering in Paramecium cells. Immunolocalization and quenched-flow correlation of time course of dephosphorylation with membrane fusion. Biochemical fournal 286, 843-849.

Hong, W. \& Lev, S. (2014). Tethering the assembly of SNARE complexes. Trends in Cell Biology 24, 35-43.

Hosein, R, E., Williams, S. A. \& Gavin, R. H. (2005). Directed motility of phagosomes in Tetrahymena thermophila requires actin and Myolp, a novel unconventional myosin. Cell Motility and the Cytoskeleton 61, 49-60.

Howe, C. J. \& Purton, S. (2007). The little genome of apicomplexan plastids: it raison d'etre and a possible explanation for the 'delayed death' phenomenon. Protist 158, $121-133$

Hunter, T. (2014). The genesis of tyrosine phosphorylation. Cold Spring Harbor Perspectives in Biology 6, a020644.

Hurtado-Lorenzo, A., Skinner, M., El Annan, J., Futai, M., Sun-Wada, G. H., Bourgoin, S., Casanova, J., Wildeman, A., Bechoua, S., Ausiello, D. A., Brown, D. \& Marshansky, V. (2006). V-ATPase interacts with ARNO and Arf6 in early endosomes and regulates the protein degradative pathway. Nature Cell Biology 8, 124-136.

Husser, M. R., Hardt, M., Blanchard, M. P., Hentschel, J., Klauke, N. \& Plattner, H. (2004). One-way calcium spill-over during signal transduction in Paramecium cells: from the cell cortex into cilia, but not in the reverse direction. Cell Calcium 36, 349-358.

Hutton, J. G. (1997). Tetrahymena: the key to the genetic analysis of the regulated pathway of polypeptide secretion? Proceedings of the National Academy of Sciences of the United States of America 94, 10490-10492.

INAGAKI, N., ITo, M., NAKANo, T. \& INAGAKI, M. (1994). Spatiotemporal distribution of protein kinase and phosphatase activities. Trends in Biochemical Sciences 19 , $448-452$.

Ishida, M., Aihara, M. S., Allen, R. D. \& FoK, A. K. (2011). The native structure of cytoplasmic dynein at work translocating vesicles in Paramecium. European fournal of Cell Biology 90, 81-92.

Ishida, T., Tabata, R., Yamada, M., Aida, M., Mitsumasu, K., Fujiwara, M., Yamaguchi, K., Shigenobu, S., Higuchi, M., Tsuji, H., Shimamoto, K., Hasebe, M. \& Sawa, S. (2014). Heterotrimeric G proteins control stem cell proliferation through CLAVATA signaling in Arabidopsis. European Molecular Biology Organisation Reports 15, 1202-1209.

Iwamoto, M. \& Allen, R. D. (2004). Uptake and rapid transfer of fluorescent ceramide analogues to acidosomes (late endosomes) in Paramecium. Fournal of Histochemistry and Cytochemistry 52, 557-565.

Imamoto, M., Koujin, T., Osakada, H., Mori, C., Kojidani, T., Matsuda, A., Asakawa, H., Hiraoka, Y. \& Haraguchi, T. (2015). Biased assembly of the nuclear pore complex is required for somatic and germline nuclear differentiation in Tetrahymena. Foumal of Cell Science 128, 1812-1823.

Iwamoto, M., Mori, G., Kojidani, T., Bunai, F., Hori, T., Fukagawa, T., Hiraoka, Y. \& Haraguchi, T. (2009). Two distinct repeat sequences of Nup98 nucleoporins characterize dual nuclei in the binucleaed ciliate Tetrahymena. Current Biology 19, 843-847.

Jacobs, M. E., DeSouza, L. V., Samaranayake, H., Pearlman, R. E., Siu, K. W. \& KLobutcher, L. A. (2006). The Tetrahymena thermophila phagosome proteome. Eukaryotic Cell 5, 1990-2000.

Jaconi, M. E., Lew, D. P., Carpentier, J. L., Magnusson, K. E., SJogren, M. \& Stendahl, O. (1990). Cytosolic free calcium elevation mediates the phagosome-lysosome fusion during phagocytosis in human neutrophils. Foumal of Cell Biology 110, 1555-1564.

Jahn, R. \& Fasshauer, D. (2012). Molecular machines governing exocytosis of synaptic vesicles. Nature 490, 201-207.

JAHN, R. \& SCHELLER, R. H. (2006). SNAREs - engines for membrane fusion. Nature Revieves Molecular Cell Biology 7, 631-643.

Jamora, C., Takizawa, P. A., Zaarour, R. F., Denesvre, C., Faulkner, D. J. \& Malhotra, V. (1997). Regulation of Golgi structure through heterotrimeric G proteins. Cell 91, 617-626.

Jauker, F., Lades, S. \& Nowack, T. (1986). The energy budget of Tetrahymena and the material fluxes into and out of the adenylate pool. Experimental Cell Research 166, $161-170$.

Jerka-Dziadosz, M. \& Frankel, J. (2014). In memorian: Krystyna Golinska (1933-2013). Fournal of Eukaryotic Microbiology 61, 328-332.

Jerka-Dziadosz, M., Koll, F., Wloga, D., Gogendeau, D., Garreau de Loubresse, N., Ruiz, F., Fabczak, S. \& Beisson, J. (2013). A centrin3-dependent, transient, appendage of the mother basal body guides the positioning of the daughter basal body in Paramecium. Protist 164, 352-368.

Jin, T. (2011). GPCR-controlled chemotaxis in Dictyostelium discoideum. Wiley Interdisciplinary Revieres. Systems Biology and Medicine 3, 717-727.

Johnson, L. N. (2009). The regulation of protein phosphorylation. Biochemical Society Transactions $37,627-641$

Juergensmeyer, E. B. (1969). Serotype expression and transformation in Tetrahymena pyriformis. Fournal of Protozoology 16, 344-352. 
Kamer, K. J. \& MoотнA, V. K. (2014). MICU1 and MICU2 play nonredundant roles in the regulation of the mitochondrial calcium uniporter. European Molecular Biology Organisation Reports 15, 299-307.

KAPOOR, P. \& SHEN, X. (2014). Mechanisms of nuclear actin in chromatin-remodeling complexes. Trends in Cell Biology 24, 238-246.

Karunakaran, S., Sasser, T., Rajalekshmi, S. \& Fratti, R. A. (2012). SNAREs, HOPS and regulatory lipids control the dynamics of vacuolar actin during homotypic fusion in S. cerevisiae. Fournal of Cell Science 125, 1683-1692.

Katritch, V., Cherezov, V. \& Stevens, R. C. (2013). Structure-function of the G protein-coupled receptor superfamily. Annual Review of Pharmacology and Toxicology 53, 531-556.

Kawasaki-Nishi, S., Bowers, K., Nishi, T., Forgac, M. \& Stevens, T. H. (2001). The amino-terminal domain of the vacuolar proton-translocating ATPase a subunit controls targeting and in vivo dissociation, and the carboxyl-terminal domain affects coupling of proton transport and ATP hydrolysis. Fournal of Biological Chemistry 276, $47411-47420$.

Kerboeuf, D. \& Cohen, J. (1990). A Ca ${ }^{2+}$ influx associated with exocytosis is specifically abolished in a Paramecium exocytotic mutant. Fournal of Cell Biology 111, $2527-2535$.

Kerboeuf, D., Le Berre, A., Dedieu, J. C. \& Cohen, J. (1993). Calmodulin is essential for assembling links necessary for exocytotic membrane fusion in Paramecium. European Molecular Biology Organisation fournal 12, 3385-3390.

Kersken, H., Momayezi, M., Braun, C. \& Plattner, H. (1986). Filamentous actin in Paramecium cells: functional and structural changes correlated with phalloidin affinity labeling in vivo. Foumal of Histochemistry and Cytochemistry 34, 455-465.

Keryer, G., Davis, F. M., Rao, P. N. \& Beisson, J. (1987). Protein phosphorylation and dynamics of cytoskeletal structures associated with basal bodies in Paramecium. Cell Motility and the Cytoskeleton 8, 44-54.

Khan, A. R. \& Ménétrey, J. (2013). Structural biology of Arf and Rab GTPases' effector recruitment and specificity. Structure 21, 1284-1297.

Kim, S. \& Dynlacht, B. D. (2013). Assembling a primary cilium. Current Opinion in Cell Biology 25, 506-511.

Kim, M. Y., Kuruvilla, H. G., Raghu, S. \& Hennessey, T. M. (1999). ATP reception and chemosensory adaptation in Tetrahymena thermophila. Fournal of Experimental Biology 202, 407-416.

Kim, K., Messinger, L. A. \& NeLson, D. L. (1998). Ca ${ }^{2+}$-dependent protein kinases of Paramecium - cloning provides evidence of a multigene family. European fournal of Biochemistry 251, 605-612.

Kissmehl, R., Froissard, M., Plattner, H., Momayezi, M. \& Cohen, J. (2002). NSF regulates membrane traffic along multiple pathways in Paramecium. Fournal of Cell Science 115, 3935-3946.

Kissmehl, R., Schilde, C., Wassmer, T., Danzer, C., Nuehse, K., Lutter, K. \& Plattner, H. (2007). Molecular identification of 26 syntaxin genes and their assignment to the different trafficking pathways in Paramecium. Trafic 8, 523-542.

Kissmehl, R., Sehring, I. M., Wagner, E. \& Plattner, H. (2004). Immunolocalization of actin in Paramecium cells. Foumal of Histochemistry and Cytochemistry 52, 1543-1559.

Kissmehl, R., Treptau, T., Hofer, H. W. \& Plattner, H. (1996). Protein phosphatase and kinase activities possibly involved in exocytosis regulation in Paramecium tetraurelia. Biochemical Fournal 317, 65-76.

Kissmehl, R., Treptau, T., Kottwitz, B. \& Plattner, H. (1997). Occurrence of a para-nitrophenyl phosphate-phosphatase with calcineurin-like characteristics in Paramecium tetraurelia. Archives of Biochemistry and Biophysics 344, 260-270.

Kiy, T., Vosskühler, C., Rasmussen, L. \& Tiedtke, A. (1993). Three pools of lysosomal enzymes in Tetrahymena thermophila. Experimental Cell Research 205, 286-292.

Klauke, N., Kissmehl, R., Plattner, H., Haga, N. \& Watanabe, T. (1998). An exocytotic mutant of Paramecium caudatum: membrane fusion without secretory contents release. Cell Calcium 23, 349-360.

Klauke, N. \& Plattner, H. (1997). Imaging of $\mathrm{Ca}^{2+}$ transients induced in Paramecium cells by a polyamine secretagogue. Fournal of Cell Science 110, 975-983.

Klauke, N. \& Plattner, H. (2000). "Frustrated exocytosis" - a novel phenomenon: membrane fusion without contents release, followed by detachment and reattachment of dense core vesicles in Paramecium cells. Fournal of Membrane Biology 176, 237-248.

Klobutcher, L. A. \& Herrick, G. (1995). Consensus inverted terminal repeat sequence of Paramecium IESs: resemblance to termini of Tc1-related and Euplotes Tec transposons. Nucleic Acids Research 23, 2006-2013.

Kloepper, T. H., Kienle, C. N. \& Fasshauer, D. (2008). SNAREing the basis of multicellularity: consequences of protein family expansion during evolution. Molecular Biology of the Cell 25, 2055-2068.

Klöppel, C., Müller, A., Marker, S. \& Simon, M. (2009). Two isoforms of eukaryotic phospholipase $\mathrm{C}$ in Paramecium affecting transport and release of GPI-anchored proteins in vivo. European Fournal of Cell Biology 88, 577-592.

Klumpr, S., Cohen, P. \& Schultz, J. E. (1990). Okadaic acid, an inhibitor of protein phosphatase 1 in Paramecium, causes sustained $\mathrm{Ca}^{2+}$-dependent backward swimming in response to depolarizing stimuli. European Molecular Biology Organisation fournal 9, 685-689.
Knochel, M., Kissmehl, R, Wissmann, J. D., Momayezi, M., Hentschel, J., Plattner, H. \& Burgoyne, R. D. (1996). Annexins in Paramecium cells. Involvement in site-specific positioning of secretory organelles. Histochemistry and Cell Biology 105, 269-281.

Knoll, G., Braun, C. \& Plattner, H. (1991a). Quenched flow analysis of exocytosis in Paramecium cells: time course, changes in membrane structure, and calcium requirements revealed after rapid mixing and rapid freezing of intact cells. Foumal of Cell Biology 113, 1295-1304.

Knoll, G., Haacke-bell, B. \& Plattner, H. (1991b). Local trichocyst exocytosis provides an efficient escape mechanism for Paramecium cells. European Foumal of Protistology 27, 381-385.

Knoll, G., Kerboeuf, D. \& Plattner, H. (1992). A rapid calcium influx during exocytosis in Paramecium cells is followed by a rise in cyclic GMP within $1 \mathrm{~s}$. Federation of European Biological Sciences Letters 304, 265-268.

Kodama, M. \& Fujishima, M. (2010). Induction of secondary symbiosis between the ciliate Paramecium and the green alga Chlorella. In Current Research, Technology and Education Topics in Applied Microbiology and Microbial Biotechnology (ed. A. MÉndez-Vilas), pp. 95-102. Formatex Research Center, Badajoz.

Kodama, Y., Suzuki, H., Dohra, H., SugiI, M., Kitazume, T., Yamaguchi, K., Shigenobu, S. \& Fujishima, M. (2014). Comparison of gene expression of Paramecium bursaria with and without Chlorella variabilis symbionts. BMC Genomics 15, 183

Krause, M. \& BrÄUcker, R. (2009). Gravitaxis of Bursaria truncatella: electrophysiological and behavioural analyses of a large ciliate cell. European fournal of Protistology 45, 98-111.

Krause, M., Bräucker, R. \& Hemmersbach, R. (2010). Gravikinesis in Stylonychia mytilus is based on membrane potential changes. Fournal of Experimental Biology 213, $161-171$.

Krishnan, A., Almen, M. S., Fredriksson, R. \& Schioth, H. B. (2012). The origin of GPCRs: identification of mammalian like rhodopsin, adhesion, glutamate and frizzled GPCRs in fungi. PLoS One 7, e29817.

Kudo, S., Muto, Y., Inagaki, M., Hidaka, H. \& Nozawa, Y. (1985a). Interaction of calcium-binding proteins with calmodulin-dependent guanylate cyclase in Tetrahymena plasma membrane. Comparative Biochemistry and Physiology. B 80, 495-498.

Kudo, S., Muto, Y. \& Nozawa, Y. (1985b). Regulation by calcium of hormoneinsensitive adenylate cyclase and calmodulin-dependent guanylate cyclase in Tetrahymena plasma membrane. Comparative Biochemistry and Physiology. B 80, 813-816.

Kuipers, A.J., Middelbeek, J. \& van Leeuwen, F. N. (2012). Mechanoregulation of cytoskeletal dynamics by TRP channels. European fournal of Cell Biology 91, 834-846.

Kumar, S., Briguglio, J. S. \& Turkewitz, A. P. (2014). An aspartyl cathepsin, CTH3, is essential for proprotein processing during secretory granule maturation in Tetrahymena thermophila. Molecular Biology of the Cell 25, 2444-2460.

Kumar, U. \& Saier, M. H. Jr. (2015). Comparative genomic analysis of integral membrane transport proteins in ciliates. Foumal of Eukaryotic Microbiology 62, 167-187.

Kung, C., Martinac, B. \& Sukharev, S. (2010). Mechanosensitive channels in microbes. Annual Review of Microbiology 64, 313-329.

Kung, G., Preston, R. R., Maley, M. E., Ling, K. Y., Kanabrocki, J. A. Seavey, B. R. \& Saimi, Y. (1992). In vivo Paramecium mutants show that calmodulin orchestrates membrane responses to stimuli. Cell Calcium 13, 413-425.

Kung, C. \& Saimi, Y. (1982). The physiological basis of taxes in Paramecium. Annual Review of Physiology 44,519-534.

Kusch, J. \& Heckmann, K. (1992). Isolation of the lembadion-factor, a morphogenetically active signal, that induces Euplotes cells to change from their ovoid form into a larger lateral winged morph. Developmental Genetics 13, 241-246.

Kussmann, M., Hauser, K., Kissmehl, R., Breed, J., Plattner, H. \& RoepstorfF, P. (1999). Comparison of in vivo and in vitro phosphorylation of the exocytosis-sensitive protein PP63/parafusin by differential MALDI mass spectrometric peptide mapping. Biochemistry 38, 7780-7790.

Kutomi, O., Hori, M., Ishida, M., Tominaga, T., Kamachi, H., Koll, F., Cohen, J., Yamada, N. \& Noguchi, M. (2012). Outer dynein arm light chain 1 is essential for controlling the ciliary response to cyclic AMP in Paramecium tetraurelia. Eukaryotic Cell 11, 645-653.

Lacy, P. E., Howell, S. L., Young, D. A. \& Fink, C. J. (1968). New hypothesis of insulin secretion. Nature 219, 1177-1179.

Ladenburger, E. M., Korn, I., Kasielke, N., Wassmer, T. \& Plattner, H. (2006). An Ins $(1,4,5) \mathrm{P}_{3}$ receptor in Paramecium is associated with the osmoregulatory system. Fournal of Cell Science 119, 3705-3717.

Ladenburger, E. M. \& Plattner, H. (2011). Calcium-release channels in Paramecium. Genomic expansion, differential positioning and partial transcriptional elimination. PLoS One 6, e27111.

Ladenburger, E. M. Sehring, I. M., Korn, I \& Plattner, H. (2009). Nove types of $\mathrm{Ca}^{2+}$ release channels participate in the secretory cycle of Paramecium cells. Molecular and Cellular Biology 29, 3605-3622.

Lai, M. M., Hong, J. J., Ruggiero, A. M., Burnett, P. E., Sleprev, V. I., De Camilli, P. \& SNyder, S. H. (1999). The calcineurin-dynamin 1 complex as a calcium sensor for synaptic vesicle endocytosis. Journal of Biological Chemistry 274, 25963-25966. 
Lampert, T. J., Coleman, K. D. \& Hennessey, T. M. (2011). A knockout mutation of a constitutive GPCR in Tetrahymena decreases both G-protein activity and chemoattraction. PLoS One 6, e28022.

Langenick, J., Araki, T., Yamada, Y. \& Williams, J. G. (2008). A Dictyostelium homologue of the metazoan $\mathrm{Cbl}$ proteins regulates STAT signalling. Fournal of Cell Science 121, 3524-3530.

Lanner, J. T., Georgiou, D. K., Joshi, A. D. \& Hamilton, S. L. (2010). Ryanodine receptors: structure, expression, molecular details, and function in calcium release. Cold Spring Harbor Perspectives in Biology 2, a003996.

Lapatsina, L., Brand, J., Poole, K., Daumke, O. \& Lewin, G. R. (2012). Stomatin-domain proteins. European fournal of Cell Biology 91, 240-245.

LASS, S. \& SpAAK, P. (2003). Chemically induced anti-predator defences in plankton: a review. Hydrobiologia 491, 221-239.

LeE, H. C. (2012). Cyclic ADP-ribose and nicotinic acid adenine dinucleotide phosphate (NAADP) as messengers for calcium mobilization. Fournal of Biological Chemistry 287, 31633-31640.

Leeck, C. L. \& Forney, J. D. (1996). The 5' coding region of Paramecium surface antigen genes controls mutually exclusive transcription. Proceedings of the National Academy of Sciences of the United States of America 93, 2838-2843.

LEICK, V. (1992). Chemotactic properties, cellular-binding and uptake of peptides and peptide derivatives - studies with Tetrahymena thermophila. Fournal of Cell Science 103, $565-570$.

Leick, V. \& Lindemose, S. (2007). Chemokinesis by Tetrahymena in response to bacterial oligopeptides. Fournal of Eukaryotic Microbiology 54, 271-274.

Leondaritis, G., Sarri, T., Dafnis, I., Efstathiou, A. \& Galanopoulou, D. (2011). Biochemical and genetic evidence for the presence of multiple phosphatidylinositol- and phosphatidylinositol 4,5-bisphosphate-specific phospholipases C in Tetrahymena. Eukaryotic Cell 10, 412-422.

Leondaritis, G., Siokos, J., Skaripa, I. \& Galanopoulou, D. (2013). Genome-wide analysis of the phosphoinositide kinome from two ciliates reveals novel evolutionary links for phosphoinositide kinases in eukaryotic cells. PLoS One $\mathbf{8}$, e78848.

Levitan, I. B. (1999). It is calmodulin after all! Mediator of the calcium modulation of multiple ion channels. Neuron 22, 645-648.

LI, R. \& Gundersen, G. G. (2008). Beyond polymer polarity: how the cytoskeleton builds a polarized cell. Nature Reviewes Molecular Cell Biology 9, 860-873.

Li, S., Yin, L., Cole, E. S., Udani, R. A. \& Karrer, K. M. (2006). Progeny of germ line knockouts of ASI2, a gene encoding a putative signal transduction receptor in Tetrahymena thermophila, fail to make the transition from sexual reproduction to vegetative growth. Developmental Biology 295, 633-646.

Liebl, D. \& Griffiths, G. (2009). Transient assembly of F-actin by phagosomes delays phagosome fusion with lysosomes in cargo-overloaded macrophages. Fournal of Cell Science 122, 2935-2945.

Lima, W. C., Balestrino, D., Forestier, C. \& Cosson, P. (2014). Two distinct sensing pathways allow recognition of Klebsiella pneumoniae by Dictyostelium amoebae. Cellular Microbiology 16, 311-323.

Linder, J. U., Engel, P., Reimer, A., Krüger, T., Plattner, H., Schultz, A. \& Schultz, J. E. (1999). Guanylyl cyclases with the topology of mammalian adenylyl cyclases and an N-terminal P-type ATPase-like domain in Paramecium, Tetrahymena and Plasmodium. European Molecular Biology Organisation fournal 18, 4222-4232.

Linkner, J., Witte, G., Zhao, H., Junemann, A., Nordholz, B., Runge-Wollmann, P., Lappalainen, P. \& Faix, J. (2014). The inverse BaR domain protein IBARa drives membrane remodeling to control osmoregulation, phagocytosis and cytokinesis. Fournal of Cell Science 127, 1279-1292.

Liv, B. A., Shah, E., Jablonowski, K., Stergachis, A., Engelmann, B. \& Nash, P. D. (2011). The SH2 domain-containing proteins in 21 species establish the provenance and scope of phosphotyrosine signaling in eukaryotes. Science Signaling $\mathbf{4}$, ra83.

López-Montero, I., Monroy, F., Velez, M. \& Devaux, P. F. (2010). Ceramide: from lateral segregation to mechanical stress. Biochimica et Biophysica Acta 1798, $1348-1356$.

Lourido, S. \& Moreno, S. N. J. (2015). The calcium signalling toolkit of the apicomplexan parasites Toxoplasma gondii and Plasmodium spp. Cell Calcium 57, $186-193$.

Lovett, J. L., Marchesini, N., Moreno, S. N. \& Sibley, L. D. (2002). Toxoplasma gondii microneme secretion involves intracellular $\mathrm{Ca}^{2+}$ release from inositol 1,4,5-trisphosphate $\mathrm{IP}_{3} /$ ryanodine-sensitive stores. Foumal of Biological Chemistry 277 , $25870-25876$.

Lu, E. \& Wolfe, J. (2001). Lysosomal enzymes in the macronucleus of Tetrahymena during its apoptosis-like degradation. Cell Death and Differentiation 8, 289-297.

Luca, F. C., Mody, M., Kurischko, C., Roof, D. M., Giddings, T. H. \& Winey, M. (2001). Saccharomyces cerevisiae Moblp is required for cytokinesis and mitotic exit. Molecular and Cellular Biology 21, 6972-6983.

Lumpert, G. J., Glas-Albrecht, R., Eisenmann, E. \& Plattner, H. (1992). Secretory organelles of Paramecium cells (trichocysts) are not remarkably acidic compartments. Fournal of Histochemistry and Cytochemistry 40, 153-160.

Lumpert, C. J., Kersken, H. \& Plattner, H. (1990). Cell surface complexes ('cortices') isolated from Paramecium tetraurelia cells as a model system for analysing exocytosis in vitro in conjunction with microinjection studies. Biochemical fournal 269 $639-645$.

Luporini, P., Alimenti, C., Ortenzi, C. \& Vallesi, A. (2005). Giliate mating types and their specific protein pheromones. Acta Protozoologica 44, 89-101.

Luporini, P., Alimenti, C. \& Vallesi, A. (2014). Ciliate mating types and pheromones. In Cilia and Flagella, Ciliates and Flagellates (eds K. Hausmann and R. RAdEK), pp. 95-118. Schweizerbart Science Publishers, Stuttgart.

Luzio, J. P., Gray, S. R. \& Bright, N. A. (2010). Endosome-lysosome fusion. Biochemical Society Transactions 38, 1413-1416.

Lynn, D. H. (2010). The Ciliated Protozoa. Third Edition 0. Springer-Verlag, Dordrecht, Heidelberg, London, New York.

Machemer, H. (1988a). Electrophysiology. In Paramecium (ed. H.-D. Görtz), pp. 185-215. Springer-Verlag, Berlin, Heidelberg.

Machemer, H. (1988b). Motor control of cilia. In Paramecium (ed. H.-D. GörTz), pp. 216-235. Springer-Verlag, Berlin, Heidelberg.

Machemer, H. (2014). How do protists keep up?. In Cilia and Flagella, Ciliates and Flagellates (eds K. Hausmann and R. RADEK), pp. 133-146. Schweizerbart Science Publishers, Stuttgart.

Machemer, H., BräUcker, R., Machemer-Röhnisch, S., Nagel, U., Neugebauer, D. C. \& Weskamp, M. (1998). The linking of extrinsic stimuli to behaviour: roles of cilia in ciliates. European fournal of Protistology 34, 254-261.

Machemer, H. \& OGura, A. (1979). Ionic conductances of membranes in ciliated and deciliated Paramecium. Fournal of Physiology 296, 49-60.

Machemer, H. \& Teunis, P. F. M. (1996). Sensory motor coupling and motor responses. In Ciliates. Cells and Organisms (eds K. Hausmann and P. C. Bradbury), pp. 379-402. Gustav Fischer Verlag, Stuttgart.

Maihle, N. J., Dedman, J. R., Means, A. R., Chafouleas, J. G. \& Satir, B. H. (1981). Presence and indirect immunofluorescent localization of calmodulin in Paramecium tetraurelia. Fournal of Cell Biology 89, 695-699.

Malkus, P., Jiang, F. \& Schekman, R. (2002). Concentrative sorting of secretory cargo proteins into COPII-coated vesicles. Foumal of Cell Biology 159, 915-921.

Malsam, J. \& Söllner, T. H. (2011). Organization of SNAREs within the Golgi stack. Cold Spring Harbor Perspectives in Biology 3, a005249.

Manahan, C. L., Iglesias, P. A., Long, Y. \& Devreotes, P. N. (2004). Chemoattractant signaling in Dictyostelium discoideum. Annual Review of Cell and Developmental Biology 20, 223-253.

Manning, G., Young, S. L., Miller, W. T. \& Zhai, Y. (2008). The protist, Monosiga brevicollis, has a tyrosine kinase signaling network more elaborate and diverse than found in any known metazoan. Proceedings of the National Academy of Sciences of the United States of America 105, 9674-9679.

Marks, B. \& McMahon, H. T. (1998). Calcium triggers calcineurin-dependent synaptic vesicle recycling in mammalian nerve terminals. Current Biology 8, 740-749.

Marmignon, A., Bischerour, J., Silve, A., Fojcik, C., Dubois, E., Arnaiz, O., Kapusta, A., Malinsky, S. \& Bétermier, M. (2014). Ku-mediated coupling of DNA cleavage and repair during programmed genome rearrangements in the ciliate Paramecium tetraurelia. PLoS Genetics 10(8), e1004552.

Martens, S. \& McMahon, H. T. (2008). Mechanisms of membrane fusion: disparate players and common principles. Nature Revieres Molecular Cell Biology 9, 543-556.

Matsuda, A. \& Forney, J. D. (2005). Analysis of Paramecium tetraurelia A-51 surface antigen gene mutants reveals positive-feedback mechanisms for maintenance of expression and temperature-induced activation. Eukaryotic Cell 4, 1613-1619.

Matsuda, A. \& Forney, J. D. (2006). The SUMO pathway is developmentally regulated and required for programmed DNA elimination in Paramecium tetraurelia. Eukaryotic Cell 5, 806-815.

Matsuda, A., Shieh, A. W., Chalker, D. L. \& Forney, J. D. (2010). The conjugation-specific Die 5 protein is required for development of the somatic nucleus in both Paramecium and Tetrahymena. Eukaryotic Cell 9, 1087-1099.

Matt, H., Bilinski, M. \& Plattner, H. (1978). Adenosinetriphosphate, calcium and temperature requirements for the final steps of exocytosis in Paramecium cells. Journal of Cell Science 32, 67-86.

Mellman, I. (1992). The importance of being acid: the role of acidification in intracellular membrane traffic. Fournal of Experimental Biology 172, 39-45.

Merlini, L., Dudin, O. \& Martin, S. G. (2013). Mate and fuse: how yeast cells do it. Open Biology 3 (doi: 10.1098/rsob.130008).

Miao, W., Xiong, J., Bowen, J., Wang, W., Liu, Y., Braguinets, O., Grigull, J., Pearlman, R. E., Orias, E. \& Gorovsky, M. A. (2009). Microarray analyses of gene expression during the Tetrahymena thermophila life cycle. PLoS One 4, e4429.

Michaelson, D., Ahearn, I., Bergo, M., Young, S. \& Philips, M. (2002). Membrane trafficking of heterotrimeric $\mathrm{G}$ proteins via the endoplasmic reticulum and Golgi. Molecular Biology of the Cell 13, 3294-3302.

Michell, R. H. (2013). Inositol lipids: from an archaeal origin to phosphatidylinositol 3,5-bisphosphate faults in human disease. Federation of European Biological Societies Fournal 280, 6281-6294

Michibata, J., Okazaki, N., Motomura, S., Uda, K., Fujiwara, S. \& Suzuki, T. (2014). Two arginine kinases of Tetrahymena pyriformis: characterization and localization. Comparative Biochemistry and Physiology, Part B 171, 34-41.

Miglietta, L. A. \& Nelson, D. L. (1988). A novel cGMP-dependent protein kinase from Paramecium. Fournal of Biological Chemistry 263, 16096-16105. 
Mim, C. \& Unger, V. M. (2012). Membrane curvature and its generation by BAR proteins. Trends in Biochemical Sciences 37, 526-533.

Mimikakis, J. L., Nelson, D. L. \& Preston, R. R. (1998). Oscillating response to a purine nucleotide disrupted by mutation in Paramecium tetraurelia. Biochemical fournal $330,139-147$.

Min, S. W., Chang, W. P. \& Südhof, T. C. (2007). E-syts, a family of membranous $\mathrm{Ca}^{2+}$-sensor proteins with multiple $\mathrm{C} 2$ domains. Proceedings of the National Academy of Sciences of the United States of America 104, 3823-3828.

Mishra, A., Eathiraj, S., Corvera, S. \& Lambright, D. G. (2010). Structural basis for Rab GTPase recognition and endosome tethering by the $\mathrm{C} 2 \mathrm{H} 2$ zinc finger of early endosomal autoantigen 1 (EEA1). Proceedings of the National Academy of Sciences of the United States of America 107, 10866-10871.

Miwa, I. \& WADA, T. (1995). Light-pulses and injection of Ip3 induce mating ability in Paramecium bursaria. Fournal of Experimental Zoology 272, 338-344.

Mizuno-Yamasaki, E., Rivera-Molina, F. \& Novick, P. (2012). GTPase networks in membrane traffick. Annual Review of Biochemistry 81, 637-659.

Mogami, Y., Yamane, A., Gino, A. \& Baba, S. A. (2004). Bioconvective pattern formation of Tetrahymena under altered gravity. Fournal of Experimental Biology 207, 3349-3359.

Mohamed, I., Husser, M., Sehring, I., Hentschel, J., Hentschel, G. \& Plattner, H. (2003). Refilling of cortical calcium stores in Paramecium cells: in situ analysis in correlation with store-operated calcium influx. Cell Calcium 34, 87-96.

Momayezi, M., Habermann, A. W., Sokolova, J. J., Kissmehl, R. \& Plattner, H. (1993). Ultrastructural and antigenic preservation of a delicate structure by cryopreparation: identification and immunogold localization during biogenesis of a secretory component (membrane-matrix connection) in Paramecium trichocysts. Journal of Histochemistry and Cytochemistry 41, 1669-1677.

Momayezi, M., Kersken, H., Gras, U., Vilmart-Seuwen, J. \& Plattner, H. (1986). Calmodulin in Paramecium tetraurelia: localization from the in vivo to the ultrastructural level. Fournal of Histochemistry and Cytochemistry 34, 1621-1638.

Momayezi, M., Kissmehl, R. \& Plattner, H. (2000). Quantitative immunogold localization of protein phosphatase 2B (calcineurin) in Paramecium cells. Fournal of Histochemistry and Cytochemistry 48, 1269-1281.

Momayezi, M., Lumpert, C. J., Kersken, H., Gras, U., Plattner, H., Krinks, M. H. \& KLEE, C. B. (1987). Exocytosis induction in Paramecium tetraurelia cells by exogenous phosphoprotein phosphatase in vivo and in vitro: possible involvement of calcineurin in exocytotic membrane fusion. Fournal of Cell Biology 105, 181-189.

Momayezi, M., Wloga, D., Kissmehl, R., Plattner, H., Jung, G., Klumpp, S. \& Schultz, J. E. (1996). Immunolocalization of protein phosphatase type 1 in Paramecium cells using antibodies against recombinant protein and peptides. Fournal of Histochemistry and Cytochemistry 44, 891-905.

Mooren, O. L., Galletta, B. J. \& Cooper, J. A. (2012). Roles for actin assembly in endocytosis. Annual Review of Biochemistry 81, 661-686.

Morgan, A. J. \& Galione, A. (2014). Two-pore channels (TPCs): current controversies. Bioessays 36, 173-183.

Müller, S., Diederichs, K., Breed, J., Kissmehl, R., Hauser, K., Plattner, H. \& Welte, W. (2002). Crystal structure analysis of the exocytosis-sensitive phosphoprotein, pp63/parafusin (phosphoglucomutase), from Paramecium reveals significant conformational variability. Fournal of Molecular Biology 315, 141-153.

Munnik, T. \& Testerink, C. (2009). Plant phospholipid signaling: "in a "nutshell". Journal of Lipid Research 50(Suppl), S260-S265.

Nair, S., Guerra, C. \& Satir, P. (1999). A Sec7-related protein in Paramecium. Federation of the American Societies of Experimental Biology fournal 13, 1249-1257.

NaITOH, Y. \& EckerT, R. (1969). Ionic mechanisms controlling behavioral responses of Paramecium to mechanical stimulation. Science 164, 963-965.

Naitoh, Y. \& Kaneko, H. (1972). Reactivated triton-extracted models of Paramecium: modification of ciliary movement by calcium ions. Science 176, 523-524.

NAKAOKA, Y., TANAKa, H. \& OosaWA, F. (1984). $\mathrm{Ca}^{2+}$-dependent regulation of beat frequency of cilia in Paramecium. Fournal of Cell Science 65, 223-231.

Nasir, A. M., Yang, Q., Chalker, D. L. \& Forney, J. D. (2015). SUMOylation is developmentally regulated and required for cell pairing during conjugation in Tetrahymena thermophila. Eukaryotic Cell 14, 170-181.

Neher, E. (1998a). Usefulness and limitations of linear approximations to the understanding of $\mathrm{Ca}^{++}$signals. Cell Calcium 24, 345-357.

NeHER, E. (1998b). Vesicle pools and $\mathrm{Ca}^{2+}$ microdomains: new tools for understanding their roles in neurotransmitter release. Neuron 20, 389-399.

Noguchi, M., Sawada, T. \& Akazawa, T. (2001). ATP-regenerating system in the cilia of Paramecium caudatum. Fournal of Experimental Biology 204, 1063-1071.

Noireaud, J. \& ANDriantsitohaina, R. (2014). Recent insights in the paracrine modulation of cardiomyocyte contractility by cardiac endothelial cells. BioMed Research International 2014 (doi: 10.1155/2014/923805).

Norberg, E., Orrenius, S. \& Zhivotovsky, B. (2010). Mitochondrial regulation of cell deatch: processing of apoptosis-inducing factor (AIF). Biochemical and Biophysical Research Communications 396, 95-100.

Novick, P. \& Zerial, M. (1997). The diversity of Rab proteins in vesicle transport. Current Opinion in Cell Biology 9, 496-504.

Nowacki, M. \& Landweber, L. F. (2009). Epigenetic inheritance in ciliates. Current Opinion in Microbiology 12, 638-643.
Nowacki, M., Shetty, K. \& Landweber, L. F. (2011). RNA-mediated epigenetic programming of genome rearrangements. Annual Review of Genomics and Human Genetics 12, 367-389.

Nowak, J. K., Gromadka, R., Juszczuk, M., Jerka-Dziadosz, M., Maliszewska, K., Mucchielli, M.-H., Gout, J.-F., Arnaiz, O., Agier, N., Tang, T., Aggerbeck, L. P., Cohen, J., Delacroix, H., Sperling, L., Herbert, C. J., Zagulski, M. \& Bétermier, M. (2011). Functional study of genes essential for autogamy and nuclear reorganization in Paramecium. Eukaryotic Cell 10, 363-372.

Ogunbayo, O. A., Zhu, Y., Rossi, D., Sorrentino, V., Ma, J., Zhu, M. X. \& Evans, A. M. (2011). Cyclic adenosine diphosphate ribose activates ryanodine receptors, whereas NAADP activates two-pore domain channels. Fournal of Biological Chemistry 286, 9136-9140.

Ogura, A. \& Machemer, H. (1980). Distribution of mechanoreceptor channels in the Paramecium surface-membrane. Fournal of Comparative Physiology 135, 233-242.

Ohno, S. (1970). Evolution by Gene Duplication. George Allen and Unwin, London.

Oldham, W. M. \& Hamm, H. E. (2008). Heterotrimeric G protein activation by G-protein-coupled receptors. Nature Reviewes Molecular Cell Biology 9, 60-71.

Orci, L., Ravazzola, M., Amherdt, M., Madsen, O., Vassalli, J. D. \& Perrelet, A. (1985). Direct identification of prohormone conversion site in insulin secreting cells. Cell 42, 671-681.

Orias, E., Cervantes, M. D. \& Hamilton, E. P. (2011). Tetrahymena thermophila, a unicellular eukaryote with separate germline and somatic genomes. Research in Microbiology 162, 578-586.

Ortenzi, C., Alimenti, C., Vallfesi, A., Di Pretoro, B., Terza, A. L. \& Luporini, P. (2000). The autocrine mitogenic loop of the ciliate Euplotes raikovi: the pheromone membrane-bound forms are the cell binding sites and potential signaling receptors of soluble pheromones. Molecular Biology of the Cell 11, 1445-1455.

Osada, E., Akematsu, T., Asano, T. \& Endoh, H. (2014). A novel mitochondrial nuclease-associated protein: a major executor of the programmed nuclear death in Tetrahymena thermophila. Biology of the Cell 106, 97-109.

Osinska, M., Wiejak, J., Wypych, E., Bilski, H., Bartosiewicz, R. \& Wyroba, E. (2011). Distinct expression, localization and function of two Rab7 proteins encoded by paralogous genes in a free-living model eukaryote. Acta Biochimica Polonica 58, 597-607.

Osipchuk, Y. \& Cahalan, M. (1992). Cell-to-cell spread of calcium signals mediated by ATP receptors in mast cells. Nature 359, 241-244.

Palade, G. E. (1975). Intracellular aspects of the process of protein synthesis. Science 189, 347-358.

Parfrey, L. W., Lahr, D. J., Knoll, A. H. \& Katz, L. A. (2011). Estimating the timing of early eukaryotric diversification with multigene molecular clocks. Proceedings of the National Academy of Sciences of the United States of America 108, 13624-13629.

Parkinson, K., Baines, A. E., Keller, T., Gruenheit, N., Bragg, L., North, R. A. \& Thompson, C. R. (2014). Calcium-dependent regulation of Rab activation and vesicle fusion by an intracellular P2X ion channel. Nature Cell Biology 16, 87-98.

Passos, A. P. \& García, C. R. (1998). Inositol 1,4,5-trisphosphate induced $\mathrm{Ca}^{2+}$ release from chloroquine-sensitive and -insensitive intracellular stores in the intraerythrocytic stage of the malaria parasite P. chabaudi. Biochemical and Biophysical Research Communications 245, 155-160.

Patel, S. \& CAI, X. (2015). Evolution of acidic $\mathrm{Ca}^{2+}$ stores and their resident $\mathrm{Ca}^{2+}$-permeable channels. Cell Calcium 57, 222-230.

Patel, S. \& Docampo, R. (2009). In with the TRP channels: intracellular functions for TRPM1 and TRPM2. Science Signaling 2, pe69.

Pearson, C. G. (2014). Choosing sides - asymmetric centriole and basal body assembly. Fournal of Cell Science 127, 2803-2810.

Peck, R. K., Swiderski, B. \& Tourmel, A.-M. (1993). Involvement of the trans-Golgi network coated vesicles, vesicle fusion, and secretory product condensation in the biogenesis of Pseudomicrothorax trichocysts. In Membrane Traffic in Protozoa (ed. H. PlattNer), pp. 1-25. JAI Press, Greenwich, London.

Pei, G., Repnik, U., Griffiths, G. \& Gutierrez, M. G. (2014). Identification of an immune-regulated phagosomal Rab cascade in macrophages. Fournal of Cell Science 127, 2071-2082.

Peters-Regehr, T., Kusch, J. \& Heckmann, K. (1997). Primary structure and origin of a predator released protein that induces defensive morphological changes in Euplotes. European Fournal of Protistology 33, 389-395.

Pfeffer, S. R. (2013). Rab GTPase regulation of membrane identity. Current Opinion in Cell Biology 25, 414-419.

Plattner, H. (2010a). How to design a highly organized cell: an unexpectedly high number of widely diversified SNARE proteins positioned at strategic sites in the ciliate, Paramecium tetraurelia. Protist 161, 497-516.

Plattner, H. (2010b). Membrane trafficking in protozoa SNARE proteins, $\mathrm{H}^{+}$-ATPase, actin, and other key players in ciliates. International Review of Cell and Molecular Biology 280, 79-184.

Plattner, H. (2013a). Contractile vacuole complex-its expanding protein inventory. International Review of Cell and Molecular Biology 306, 371-416.

Plattner, H. (2013b). The contractile vacuole complex of protists - new cues to function and biogenesis. Critical Reviews in Microbiology 41, 218-227 (doi: $10.3109 / 1040841 \times 2013.821650)$ 
Plattner, H. (2014). Calcium regulation in the protozoan model, Paramecium tetraurelia. fournal of Eukaryotic Microbiology 61, 95-114.

Plattner, H. (2015a). Calcium signalling in the ciliated protozoan model, Paramecium: strict signal localisation by epigenetically controlled positioning of different $\mathrm{Ca}^{2+}$-channels. Cell Calcium 57, 203-213.

Plattner, H. (2015b). Molecular aspects of calcium signalling at the crossroads of unikont and bikont eukaryotic evolution - the ciliated protozoan Paramecium in focus. Cell Calcium 57, 174-185.

Plattner, H., Braun, C. \& Hentschel, J. (1997). Facilitation of membrane fusion during exocytosis and exocytosis-coupled endocytosis and acceleration of "ghost" detachment in Paramecium by extracellular calcium. A quenched-flow/freeze-fracture analysis. Foumal of Membrane Biology 158, 197-208.

PlattNer, H. \& Kissmehl, R. (2003). Molecular aspects of membrane trafficking in Paramecium. International Review of Cytology 232, 185-216.

Plattner, H. \& Kissmehl, R. (2005). Molecular aspects of rapid, reversible, $\mathrm{Ca}^{2+}$-dependent de-phosphorylation of pp63/parafusin during stimulated exo-endocytosis in Paramecium cells. Cell Calcium 38, 319-327.

PlattNer, H. \& Klauke, N. (2001). Calcium in ciliated protozoa: sources, regulation, and calcium-regulated cell functions. International Review of Cytology 201, 115-208.

Plattner, H., Knoll, G. \& Pape, R. (1993). Synchronization of different steps of the secretory cycle in Paramecium tetraurelia: trichocyst exocytosis, exocytosis-coupled endocytosis and intracellular transport. In Membrane Traffic in Protozoa (ed. H. PlattNeR), pp. 123-148. JAI Press, Greenwich, London.

Plattner, H., Matt, H., Kersken, H., Haacke, B. \& Stürzl, R. (1984). Synchronous exocytosis in Paramecium cells. I. A novel approach. Experimental Cell Research 151, 6-13.

Plattner, H., Sehring, I. M., Mohamed, I. K., Miranda, K., De Souza, W., Billington, R., Genazzani, A. \& Ladenburger, E. M. (2012). Calcium signaling in closely related protozoan groups (Alveolata): non-parasitic ciliates (Paramecium, Tetrahymena) vs. parasitic Apicomplexa (Plasmodium, Toxoplasma). Cell Calcium 51, 351-382.

Plattner, H., Sehring, I. M., Schilde, C. \& Ladenburger, E. M. (2009). Pharmacology of ciliated protozoa--drug (in)sensitivity and experimental drug (ab)use. International Review of Cell and Molecular Biology 273, 163-218.

Plattner, H., Stürzl, R. \& Matt, H. (1985). Synchronous exocytosis in Paramecium cells. IV. Polyamino compounds as potent trigger agents for repeatable trigger-redocking cycles. European Fournal of Cell Biology 36, 32-37.

Plattner, H. \& Verkhratsky, A. (2012). $\mathrm{Ca}^{2+}$ signalling early in evolution - all but primitive. Fournal of Cell Science 126, 2141-2150.

Plattner, H. \& Verkhratsky, A. (2015). The ancient roots of calcium signalling evolutionary tree. Cell Calcium 57, 123-132.

Plattner, H., Westphal, C. \& Tiggemann, R. (1982). Cytoskeleton-secretory vesicle interactions during the docking of secretory vesicles at the cell membrane in Paramecium tetraurelia cells. Fournal of Cell Biology 92, 368-377.

Popoff, V., Adolf, F., Brügger, B. \& Wieland, F. (2011). COPI budding within the Golgi stack. Cold Spring Harbor Perspectives in Biology 3, a005231.

Pouphile, M., Leforttran, M., Plattner, H., Rossignol, M. \& Beisson, J. (1986). Genetic dissection of the morphogenesis of exocytosis sites in Paramecium. Biology of the Cell 56, 151-161.

Preer, J. R. Jr., Preer, L. B. \& Rudman, B. M. (1981). mRNAs for the immobilization antigens of Paramecium. Proceedings of the National Academy of Sciences of the United States of America 78, 6776-6778.

Preer, J. R. Jr., Preer, L. B., Rudman, B. \& Barnett, A. (1987). Molecular biology of the genes for immobilization antigens in Paramecium. Fournal of Protozoology 34 $418-423$.

Preston, R. R., Kink, J. A., Hinrichsen, R. D., Saimi, Y. \& Kung, C. (1991). Calmodulin mutants and $\mathrm{Ca}^{2+}$-dependent channels in Paramecium. Annual Review of Physiology 53, 309-319.

Preston, R. R., Saimi, Y. \& Kung, C. (1992a). Calcium current activated upon hyperpolarization of Paramecium tetraurelia. Fournal of General Physiology 100, 233-251.

Preston, R. R., Saimi, Y. \& Kung, C. (1992b). Calcium-dependent inactivation of the calcium current activated upon hyperpolarization of Paramecium tetraurelia. Fournal of General Physiology 100, 253-268.

Prole, D. L. \& TAylor, C. W. (2011). Identifiction of intracellular and plasma membrane calcium channel homologues in pathogenic parasites. PLoS One 6, e26218.

Prusiner, S. B. (2004). Prion Biology and Diseases. Second Edition. Cold Spring Harbor Laboratories, Cold Spring Harbor.

Pucciarelli, S., Ballarini, P., Sparvoli, D., Barchetta, S., Yu, T., Detrich, H. W. \& Miceli, C. (2012). Distinct functional roles of beta-tubulin isotypes in microtubule arrays of Tetrahymena thermophila, a model single-celled organism. PLoS One 7, e39694.

Quehenberger, O., Prossnitz, E. R., Cavanagh, S. L., Colchrane, G. G. \& YE, R. D. (1993). Multiple domains of the $\mathrm{N}$-formylpeptide receptor are required for high-affinity ligand binding. Construction and analysis of chimeric $\mathrm{N}$-formyl peptide receptors. Fournal of Biological Chemistry 268, 18167-18175.

Ramoino, P., Candiani, S., Pittaluga, A. M., Usai, G., Gallus, L., Ferrando, S., Milanese, M., Faimali, M. \& Bonanno, G. (2014). Pharmacological characterization of NMDA-like receptors in the single-celled organism Paramecium primaurelia. Fournal of Experimental Biology 217, 463-471.

Ramoino, P., Gallus, L., Beltrame, F., Diaspro, A., Fato, M., Rubini, P., Stigliani, S., Bonanno, G. \& Usai, C. (2006). Endocytosis of GABA B receptors modulates membrane excitability in the single-celled organism Paramecium. Fournal of Cell Science 119, 2056-2064.

Rangaraju, V., Calloway, N. \& Ryan, T. A. (2014). Activity-driven local ATP synthesis is required for synaptic function. Cell 156, 825-835.

Rasmussen, M. I. \& Wheatley, D. N. (2007). Purification and characterisation of cell survival factor 1 (TCSF1) from Tetrahymena thermophila. Fournal of Cell Communication and Signaling 1, 185-193.

Reuter, A. T., Stuermer, C. A. \& Plattner, H. (2013). Identification, localization, and functional implications of the microdomain-forming stomatin family in the ciliated protozoan Paramecium tetraurelia. Eukaryotic Cell 12, 529-544.

RiccARDI, D. \& KEMP, P.J. (2012). The calcium-sensing receptor beyond extracellular calcium homeostasis: conception, development, adult physiology, and disease. Annual Review of Physiology 74, 271-297.

Ricci, N. (1996). Ethology of ciliates. In Ciliates, Cells as Organisms (eds K. HausmanN and P. C. BRAdbury), pp. 403-416. Gustav Fischer Verlag, Stuttgart, Jena, New York.

Rizo, J., Chen, X. \& Arac, D. (2006). Unraveling the mechanisms of synaptotagmin and SNARE function in neurotransmitter release. Trends in Cell Biology 16, 339-350. Roberts, A. M. (2010). The mechanics of gravitaxis in Paramecium. Fournal of Experimental Biology 213, 4158-4162.

Rossi, V., Banfield, D. K., Vacca, M., Dietrich, L. E., Ungermann, C., D'Esposito, M., Galli, T. \& Filippini, F. (2004). Longins and their longin domains: regulated SNAREs and multifunctional SNARE regulators. Trends in Biochemical Sciences 29, 682-688.

Rothman, J. E. (2014). The principle of membrane fusion in the cell (Nobel lecture). Angewandte Chemie International Edition 53, 12676-12694.

Rubin, R. (1974). Calcium and the Secretory Process. Plenum Press, New York.

Rubin, R. P. (2012). Calcium and Cellular Secretion. Springer, Heidelberg, New York, London.

Ruiz, F., Beisson, J., Rossier, J. \& Dupuis-Williams, P. (1999). Basal body duplication in Paramecium requires $\gamma$-tubulin. Current Biology 9, 43-46.

Ruiz, F., Garreau de Loubresse, N., Klotz, C., Beisson, J. \& Koll, F. (2005). Centrin deficiency in Paramecium affects the geometry of basal-body duplication. Current Biology 15, 2097-2106.

Ruiz, F., Krzywicka, A., Klotz, G., Keller, A., Cohen, J., Koll, F., Balavoine, G. \& BEISSON, J. (2000). The SM19 gene, required for duplication of basal bodies in Paramecium, encodes a novel tubulin, $\eta$-tubulin. Current Biology 10, 1451-1454.

Ryals, P. E., Bae, S. \& Patterson, C. E. (1999). Evidence for early signaling events in stomatin-induced differentiation of Tetrahymena vorax. Fournal of Eukaryotic Microbiology 46, 77-83.

Saidu, S. P., Weeraratne, S. D., Valentine, M., Delay, R. \& Van Houten, J. L. (2009). Role of plasma membrane calcium ATPases in calcium clearance from olfactory sensory neurons. Chemical Senses 34, 349-358.

Saimi, Y. \& Kung, C. (1987). Behavioral genetics of Paramecium. Annual Review of Genetics 21, 47-65.

SAImi, Y. \& Kung, C. (1994). Ion channel regulation by calmodulin binding. Federation of European Biochemical Societies Letters 350, 155-158.

SAimi, Y. \& Kung, C. (2002). Calmodulin as an ion channel subunit. Annual Review of Physiology 64, 289-311.

Sainsard-Chanet, A. \& Cummings, D. (1988). Mitochondria. In Paramecium (ed. H.-D. GöRTZ), pp. 167-184. Springer-Verlag, Berlin, Heidelberg.

Salathe, M. (2007). Regulation of mammalian ciliary beating. Annual Review of Physiology 69, 401-422.

Samaranayake, H. S., Cowan, A. E. \& Klobutcher, L. A. (2011). Vacuolar protein sorting protein 13A, TtVPS13A, localizes to the Tetrahymena thermophila phagosome membrane and is required for efficient phagocytosis. Eukaryotic Cell 10, 1207-1218.

Samie, M., Wang, X., Zhang, X., Goschka, A., Li, X., Cheng, X., Gregg, E., Azar, M., Zhuo, Y., Garrity, A. G., Gao, Q., Slaugenhaupt, S., Pickel, J., Zolov, S. N., Weisman, L. S., Lenk, G. M., Titus, S., Bryant-Genevier, M., Southall, N., Juan, M., Ferrer, M. \& Xu, H. (2013). A TRP channel in the lysosome regulates large particle phagocytosis via focal exocytosis. Developmental Cell 26, 511-524.

Sancak, Y., Markhard, A. L., Kitami, T., Kovacs-Bogdan, E., Kamer, K. J., Udeshi, N. D., Carr, S. A., Chaudhuri, D., Clapham, D. E., Li, A. A., Calvo, S. E., Goldberger, O. \& Mootha, V. K. (2013). EMRE is an essential component of the mitochondrial calcium uniporter complex. Science 342, 1379-1382.

Satow, Y., Murphy, A. D. \& Kung, C. (1983). The ionic basis of the depolarizing mechanoreceptor potential of Paramecium tetraurelia. Fournal of Experimental Biology 103, 253-264.

Schilde, C., Schönemann, B., Sehring, I. M. \& Plattmer, H. (2010). Distinct subcellular localization of a group of synaptobrevin-like SNAREs in Paramecium tetraurelia and effects of silencing SNARE-specific chaperone NSF. Eukaryotic Cell 9, 288-305. 
Schilde, C., Wassmer, T., Mansfeld, J., Plattner, H. \& Kissmehl, R. (2006). A multigene family encoding R-SNAREs in the ciliate Paramecium tetraurelia. Traffic $7,440-455$.

Schon, E. A., DiMauro, S. \& Hirano, M. (2012). Human mitochondrial DNA: roles of inherited and somatic mutations. Nature Reviewes Genetics 13, 878-890.

Schultz, J. E., Grünemund, R., von Hirschhausen, R. \& Schonefeld, U. (1984). Ionic regulation of cyclic AMP levels in Paramecium tetraurelia in vivo. Federation of European Biochemical Societies Letters 167, 113-116.

Schultz, J. E. \& Klumpr, S. (1991). Calcium-regulated guanylyl cyclases from Paramecium and Tetrahymena. Methods in Enzymology 195, 466-474.

Schultz, J. E. \& Klumpr, S. (1993). Cyclic nucleotides and calcium signaling in Paramecium. Advances in Second Messenger and Phosphoprotein Research 27, 25-46.

Schultz, J. E., Pohl, T. \& Klumpr, S. (1986). Voltage-gated $\mathrm{Ca}^{2+}$ entry into Paramecium linked to intraciliary increase in cyclic-GMP. Nature 322, 271-273.

Schultz, J. E. \& Schönborn, C. (1994). Cyclic AMP formation in Tetrahymena pyriformis is controlled by a $\mathrm{K}^{+}$-conductance. Federation of European Biochemical Societies Letters 356, 322-326.

Schwartz, J. H., Li, G., Yang, Q., Suri, V., Ross, J. J. \& Alexander, E. A. (2007). Role of SNAREs and $\mathrm{H}^{+}$-ATPase in the targeting of proton pump-coated vesicles to collecting duct cell apical membrane. Kidney International 72, 1310-1315.

Sehring, I. M., Klotz, C., Beisson, J. \& Plattner, H. (2009). Rapid downregulation of the $\mathrm{Ca}^{2+}$-signal after exocytosis stimulation in Paramecium cells: essential role of a centrin-rich filamentous cortical network, the infraciliary lattice. Cell Calcium 45, 89-97.

Sehring, I. M., Mansfeld, J., Reiner, C., Wagner, E., Plattner, H. \& Kissmehl, R. (2007a). The actin multigene family of Paramecium tetraurelia. BMC Genomics $\mathbf{8}, 82$.

Sehring, I. M., Reiner, C., Mansfeld, J., Plattner, H. \& Kissmehl, R. (2007b). A broad spectrum of actin paralogs in Paramecium tetraurelia cells display differential localization and function. Fournal of Cell Science 120, 177-190.

Sehring, I. M. \& Plattner, H. (2004). $\mathrm{Ca}^{2+}$ oscillations mediated by exogenous GTP in Paramecium cells: assessment of possible $\mathrm{Ca}^{2+}$ sources. Cell Calcium 36, 409-420.

Sehring, I. M., Reiner, C. \& Plattner, H. (2010). The actin subfamily PtAct4, out of many subfamilies, is differentially localized for specific local functions in Paramecium tetraurelia cells. European Fournal of Cell Biology 89, 509-524.

SEIFERT, R. (2015). How do basic secretagogues activate mast cells? Naunyn Schmiedeberg's Archives of Pharmacology 388, 279-281

Shen, H. M. \& Mizushima, N. (2014). At the end of the autophagic road: an emerging understanding of lysosomal functions in autophagy. Trends in Biochemical Sciences 39, $61-71$.

Shen, H., Pirruccello, M. \& De Camilli, P. (2012). SnapShot: membrane curvature sensors and generators. Cell 150, 1300.e1-e2.

Sherwood, M. W., Prior, I. A., Voronina, S. G., Barrow, S. L., Woodsmith, J. D., Gerasimenko, O. V., Petersen, O. H. \& Tepikin, A. V. (2007). Activation of trypsinogen in large endocytic vacuoles of pancreatic acinar cells. Proceedings of the National Academy of Sciences of the United States of America 104, 5674-5679.

Shiozaki, N., Nakano, K., Kushida, Y., Noguchi, T. Q., Uyeda, T. O., Wloga, D., Dave, D., Vasudevan, K. K., Gaertig, J. \& Numata, O. (2013). ADF/cofilin is not essential but is critically important for actin activities during phagocytosis in Tetrahymena thermophila. Eukaryotic Cell 12, 1080-1086.

Sieber, J. J., Willig, K. I., Kutzner, C., Gerding-Reimers, C., Harke, B., Donnert, G., Rammner, B., Eggeling, C., Hell, S. W., Grubmüller, H. \& LANG, T. (2007). Anatomy and dynamics of a supramolecular membrane protein cluster. Science 317, 1072-1076.

Siegmund, L., Burmester, A., Fischer, M. S. \& Wostemeyer, J. (2013). A model for endosymbiosis: interaction between Tetrahymena pyriformis and Escherichia coli. European Fournal of Protistology 49, 552-563.

Simon, M. C. \& KUSCH, J. (2013). Communicative functions of GPI-anchored surface proteins in unicellular eukaryotes. Critical Revieres in Microbiology 39, 70-78.

Simon, M. \& Plattner, H. (2014). Unicellular eukaryotes as models in cell and molecular biology: critical appraisal of their past and future value. International Review of Cell and Molecular Biology 309, 141-198.

Simon, M. C. \& Schmidt, H. J. (2007). Antigenic variation in ciliates: antigen structure, function, expression. Fournal of Eukaryotic Microbiology 54, 1-7.

SimONS, K. \& GerL, M. J. (2010). Revitalizing membrane rafts: new tools and insights. Nature Reviewes Molecular Cell Biology 11, 688-699.

Singh, D. P., Saudemont, B., Guglielmi, G., Arnaiz, O., Goût, J. F., Prajer, M., Potekhin, A., Przybos, E., Aubusson-Fleury, A., Bhullar, S., Bouhouche, K., Lhuillier-Akakpo, M., Tanty, V., Blugeon, C., Alberti, A., Labadie, K., Aury, J. M., Sperling, L., Duharcourt, S. \& Meyer, E. (2014). Genome-defence small RNAs exapted for epigenetic mating-type inheritance. Nature 509, 447-452.

Skog, S., Tribukait, B. \& Sundius, G. (1982). Energy metabolism and ATP turnover time during the cell cycle of Ehrlich ascites tumour cells. Experimental Cell Research $141,23-29$.

Slabodnick, M. M., Ruby, J. G., Dunn, J. G., Feldman, J. L., DeRisi, J. L. \& Marshall, W. F. (2014). The kinase regulator Mobl acts as a patterning protein for Stentor morphogenesis. PLoS Biology 12(5), e1001861.
Steigh, M. A. (2014). Encounters with cilia. In Cilia and Flagella, Ciliates and Flagellates (eds K. Hausmann and R. RadeK), pp. 121-131. Schweizerbart Science Publishers, Stuttgart.

SMirlis, D. \& Soteriadou, K. (2011). Trypanosomatid apoptosis: 'apoptosis' without the canonical regulators. Virulence 2, 253-256.

Smith, D. G. S., Gawryluk, R. M. R., Spencer, D. F., Pearlman, R. E., Siu, K. W. M. \& Gray, M. W. (2007). Exploring the mitochondrial proteome of the ciliate protozoon Tetrahymena thermophila: direct analysis by tandem mass spectrometry. Fournal of Molecular Biology 374, 837-863.

Smith-Somerville, H. E., Hardman, J. K., Timkovich, R., Ray, W. J., Rose, K. E., Ryals, P. E., Gibbons, S. H. \& Buhse, H. E. Jr. (2000). A complex of iron and nucleic acid catabolites is a signal that triggers differentiation in a freshwater protozoan. Proceedings of the National Academy of Sciences of the United States of America 97, $7325-7330$.

Sogame, Y., Kojima, K., Takeshita, T., Kinoshita, E. \& Matsuoka, T. (2014). Identification of differentially expressed water-insoluble proteins in the encystment process of Colpoda cucullus by two-dimensional electrophoresis and LC-MS/MS analysis. Fournal of Eukaryotic Microbiology 61, 51-60.

Sonneborn, T. M. (1949). Beyond the gene. American Scientist 37, 33-59.

SPERling, L. (2011). Remembrance of things past retrieved from the Paramecium genome. Research in Microbiology 162, 587-597.

Sperling, L., Keryer, G., Ruiz, F. \& Beisson, J. (1991). Cortical morphogenesis in Paramecium: a transcellular wave of protein phosphorylation involved in ciliary rootlet disassembly. Developmental Biology 148, 205-218.

Srivastava, M., Simakov, O., Chapman, J., Fahey, B., Gauthier, M. E., Mitros, T., Richards, G. S., Conaco, C., Dacre, M., Hellsten, U., Larroux, C., Putnam, N. H., Stanke, M., Adamska, M., Darling, A., Degnan, S. M., Oakley, T. H., Plachetzki, D. C., Zhai, Y., Adamski, M., Calcino, A., Cummins, S. F., Goodstein, D. M., Harris, C., Jackson, D. J., Leys, S. P., Shu, S., Woodcroft, B. J., Vervoort, M., Kosik, K. S., Manning, G., Degnan, B. M. \& Rokhsar, D. S. (2010). The Amphimedon queenslandica genome and the evolution of animal complexity. Nature 466, 720-726.

STEiner, D. F. (1998). The proprotein convertases. Current Opinion in Chemical Biology 2, 37-39.

Stemm-Wolf, A. J., Meehl, J. B. \& Winey, M. (2013). Sfr13, a member of a large family of asymmetrically localized Sfil-repeat proteins, is important for basal body separation and stability in Tetrahymena thermophila. Fournal of Cell Science $\mathbf{1 2 6}$ $1659-1671$.

Stemm-Wolf, A. J., Morgan, G., Giddings, T. H.Jr., White, E. A., Marchione, R., McDonald, H. B. \& Winey, M. (2005). Basal body duplication and maintenance require one member of the Tetrahymena thermophila centrin gene family. Molecular Biology of the Cell 16, 3606-3619.

Stenmark, H. (2012). The Rabs: a family at the root of metazoan evolution. BMC Biology 10, 68.

Stock, C., Grønlien, H. K. \& Allen, R. D. (2002). The ionic composition of the contractile vacuole fluid of Paramecium mirrors ion transport across the plasma membrane. European fournal of Cell Biology 81, 505-515.

Stock, G., Krüppel, U. T., Key, G. \& Lueken, W. (1999). Sexual behaviour in Euplotes raikovi is accompanied by pheromone-induced modifications of ionic currents. Fournal of Experimental Biology 202, 475-483.

Strotmann, R., Schröck, K., Böselt, I., Stäubert, C., Russ, A \& SchöneberG, T. (2011). Evolution of GPCR: change and continuity. Molecular and Cellular Endocrinology 331, 170-178.

SüDHoF, T. G. (2013). Neurotransmitter release: the last millisecond in the life of a synaptic vesicle. Neuron $\mathbf{8 0}, 675-690$.

SüDHOF, T. C. (2014). The molecular machinery of neurotransmitter release (Nobel lecture). Angewandte Chemie International Edition 53, 12696-12717.

Suga, H., Torruella, G., Burger, G., Brown, M. W. \& Ruiz-Trillo, I. (2014). Earliest holozoan expansion of phosphotyrosine signaling. Molecular Biology of Evolution 31, 517-528.

Sugiura, M. \& Harumoto, T. (2001). Identification, characterization, and complete amino acid sequence of the conjugation-inducing glycoprotein (blepharmone) in the ciliate Blepharisma japonicum. Proceedings of the National Academy of Sciences of the United States of America 98, 14446-14451.

Sugiura, M., Shiotani, H., Suzaki, T. \& Harumoto, T. (2010). Behavioural changes induced by the conjugation-inducing pheromones, gamone 1 and 2 , in the ciliate Blepharisma japonicum. European fournal of Protistology 46, 143-149.

Sung, C. H. \& LERoux, M. R. (2013). The roles of evolutionarily conserved functional modules in cilia-related trafficking. Nature Cell Biology 15, 1387-1397.

Surmacz, L., Wiejak, J. \& Wyroba, E. (2006). Cloning of two genes encoding Rab7 in Paramecium. Acta Biochimica Polonica 53, 149-156.

Sutton, R. B., Fasshauer, D., Jahn, R. \& Brunger, A. T. (1998). Crystal structure of a SNARE complex involved in synaptic exocytosis at $2.4 \AA$ resolution. Nature 395, $347-353$.

Swart, E. C., Wilkes, G. D., Sandoval, P. Y., Arambasic, M., Sperling, L. \& NowACKI, M. (2014). Genome-wide analysis of genetic and epigenetic control of programmed DNA deletion. Nucleic Acids Research 42, 8970-8983. 
Tамм, S. L. (2014). Ctenophores and termites - systems for motility. In Cilia and Flagella, Ciliates and Flagellates (eds K. Hausmann and R. RadeK), pp. 147-171. Schweizerbart Science Publishers, Stuttgart.

Tani, T., Allen, R. D. \& Naitoh, Y. (2001). Cellular membranes that undergo cyclic changes in tension: direct measurement of force generation by an in vitro contractile vacuole of Paramecium multimicronucleatum. Fournal of Cell Science 114, 785-795.

Thewes, S., Schubert, S. K., Park, K. \& Mutzel, R. (2014). Stress and development in Dictyostelium discoideum: the involvement of the catalytic A subunit. fournal of Basic Microbiology 54, 607-613.

Thomas, P., Lee, A. K., Wong, J. G. \& Almers, W. (1994). A triggered mechanism retrieves membrane in seconds after $\mathrm{Ca}^{2+}$-stimulated exocytosis in single pituitary cells. Fournal of Cell Biology 124, 667-675.

Tian, M., Chen, X., Xiong, Q., Xiong, J., Xiao, G., Ge, F., Yang, F. \& Miao, W. (2014). Phosphoproteomic analysis of protein phosphorylation networks in Tetrahymena thermophila, a model single-celled organism. Molecular and Cellular Proteomics 13, 503-519.

Tiedtke, A., Hunseler, P., Florin-Christensen, J. \& Florin-Christensen, M. (1988). Exocytosis, endocytosis and membrane recycling in Tetrahymena thermophila. Fournal of Cell Science 89, 515-520.

Tomaru, A. (2002). Encystment-inducing factors in the ciliate Euplotes elegans. Zoological Science 19, 741-746.

Tominaga, T. \& Allen, R. (1998). Electrophysiology of the in situ contractile vacuole complex of Paramecium reveals its membrane dynamics and electrogenic site during osmoregulatory activity. Fournal of Experimental Biology 201, 451-460.

Treisman, R. (2013). Shedding light on nuclear actin dynamics and function. Trends in Biochemical Sciences 38, 376-377.

Underhill, D. M. \& Goodridge, H. S. (2012). Information processing during phagocytosis. Nature Reviews Immunology 12, 492-502.

Uren, A. G., O’Rourke, K., Aravind, L., Pisabarro, M. T., Seshagiri, S., Koonin, E. V. \& Dixit, V. M. (2000). Identification of paracaspases and metacaspases: two ancient families of caspase-like proteins, one of which plays a key role in MALT lymphoma. Molecular Cell 6, 961-967.

Valentine, M. S., Rajendran, A., Yano, J., Weeraratne, S. D., Beisson, J., Cohen, J., Koll, F. \& Van Houten, J. (2012). Paramecium BBS genes are key to presence of channels in cilia. Cilia $\mathbf{1}, 16$.

Vallesi, A., Ballarini, P., Di Pretoro, B., Alimenti, C., Miceli, G. \& Luporini, P. (2005). Autocrine, mitogenic pheromone receptor loop of the ciliate Euplotes raikovi: pheromone-induced receptor internalization. Eukaryotic Cell 4, 1221-1227.

Vallesi, A., Di Pretoro, B., Ballarini, P., Apone, F. \& Luporini, P. (2010). A novel protein kinase from the ciliate Euplotes raikovi with close structural identity to the mammalian intestinal and male-germ cell kinases: characterization and functional implications in the autocrine pheromone signaling loop. Protist 161, 250-263.

Vallesi, A., Giuseppe, G. D., Dini, F. \& Luporini, P. (2008). Pheromone evolution in the protozoan ciliate, Euplotes: the ability to synthesize diffusible forms is ancestral and secondarily lost. Molecular Phylogenetics and Evolution 47, 439-442.

Van Houten, J. (1998). Chemosensory transduction in Paramecium. European Fournal of Protistology 34, 301-307.

Vayssié, L., Skouri, F., Sperling, L. \& Cohen, J. (2000). Molecular genetics of regulated secretion in Paramecium. Biochimie 82, 269-288.

Verbsky, J. W. \& Turkewitz, A. P. (1998). Proteolytic processing and $\mathrm{Ca}^{2+}$-binding activity of dense-core vesicle polypeptides in Tetrahymena. Molecular Biology of the Cell 9, 497-511.

Verkhratsky, A. \& Burnstock, G. (2014). Biology of purinergic signalling: its ancient evolutionary roots, its omnipresence and its multiple functional significance. BioEssays 36, 697-705.

Vetter, D., Kissmehl, R., Treptau, T., Hauser, K., Kellermann, J. \& Plattner, H. (2003). Molecular identification of a calcium-inhibited catalytic subunit of casein kinase type 2 from Paramecium tetraurelia. Eukaryotic Cell 2 , $1220-1233$.

Vicinanza, M., D’Angelo, G., Di Campli, A. \& De Matteis, M. A. (2008). Function and dysfunction of the PI system in membrane trafficking. European Molecular Biology Organisation fournal 27, 2457-2470.

Vilmart-Seuwen, J., Kersken, H., Stürzl, R. \& Plattner, H. (1986). ATP keeps exocytosis sites in a primed state but is not required for membrane fusion: an analysis with Paramecium cells in vivo and in vitro. Fournal of Cell Biology 103, 1279-1288.

Vogt, A. \& Mochizuki, K. (2013). A domesticated PiggyBac transposase interacts with heterochromatin and catalyzes reproducible DNA elimination in Tetrahymena. PLoS Genetics 9(12), e1004032.

WAlczaK, C. E., GAYeK, S. \& OHI, R. (2013). Microtubule-depolymerizing kinesins. Annual Review of Cell and Developmental Biology 29, 417-441.

WALCZAK, C. E. \& Nelson, D. L. (1993). In vitro phosphorylation of ciliary dyneins by protein kinases from Paramecium. Fournal of Cell Science 106, 1369-1376.

Wassenberg, J. J., Clark, K. D. \& Nelson, D. L. (1997). Effect of SERCA pump inhibitors on chemoresponses in Paramecium. Fournal of Eukaryotic Microbiology 44 $574-581$.

Wassmer, T., Froissard, M., Plattner, H., Kissmehl, R. \& Cohen, J. (2005). The vacuolar proton-ATPase plays a major role in several membrane-bounded organelles in Paramecium. Fournal of Cell Science 118, 2813-2825.
Wassmer, T., Kissmehl, R., Cohen, J. \& Plattner, H. (2006). Seventeen a-subunit isoforms of Paramecium V-ATPase provide high specialization in localization and function. Molecular Biology of the Cell 17, 917-930.

Wassmer, T., Sehring, I. M., Kissmehl, R. \& Plattner, H. (2009). The V-ATPase in Paramecium: functional specialization by multiple gene isoforms. Pflugers Archiv European fournal of Physiology 457, 599-607.

Watanabe, A., Kurasawa, Y., Watanabe, Y. \& Numata, O. (1998). A new Tetrahymena actin-binding protein is localized in the division furrow. Fournal of Biochemistry 123, 607-613.

Watanabe, S., Rost, B. R., Pérez, M. C., Davis, M. W., Kielczynski, B. S., Rosenmund, D. \& Jorgensen, E. M. (2013). Ultrafast endocytosis at mouse hippocampal synapses. Nature 504, 242-247.

Weber, J. H., Vishnyakov, A., Намbach, K., Schultz, A., Schultz, J. E. \& Linder, J. U. (2004). Adenylyl cyclases from Plasmodium, Paramecium and Tetrahymena are novel ion channel/enzyme fusion proteins. Cellular Signalling 16, 115-125.

Welz, T., Wellbourne-Wood, J. \& Kerkhoff, E. (2014). Orchestration of cell surface proteins by Rab11. Trends in Cell Biology 24, 407-415.

Whiteheart, S. W., Schraw, T. \& Matveeva, E. A. (2001). N-ethylmaleimide sensitive factor (NSF) structure and function. International Review of Cytology 207, $71-112$.

WicksteAd, B. \& GULL, K. (2006). A "holistic" kinesin phylogeny reveals new kinesin families and predicts protein functions. Molecular Biology of the Cell 17, 1734-1743.

Williams, K. (1997). Interactions of polyamines with ion channels. Biochemical fournal 325, 289-297.

WLOGA, D. \& FrankEL,J. (2012). From molecules to morphology: cellular organization of Tetrahymena thermophila. Methods in Cell Biology 109, 83-140.

Wloga, D. \& Gaertig, J. (2010). Post-translational modifications of microtubules. Fournal of Cell Science 123, 3447-3455.

Wloga, D., Rogowski, K., Sharma, N., Van Dijk, J., Janke, G., Eddé, B., Bré, M. H., Levilliers, N., Redeker, V., Duan, J., Gorovsky, M. A., Jerka-Dziadosz, M. \& GAertiG, J. (2008). Glutamylation on alpha-tubulin is not essential but affects the assembly and functions of a subset of microtubules in Tetrahymena thermophila. Eukaryotic Cell 7, 1362-1372.

Wright, M. V. \& VAN Houten, J. L. (1990). Characterization of a putative $\mathrm{Ca}^{2+}$-transporting $\mathrm{Ca}^{2+}$-ATPase in the pellicles of Paramecium tetraurelia. Biochimica et Biophysica Acta 1029, 241-251.

Wu, L. G., Hamid, E., Shin, W. \& Chiang, H. C. (2014). Exocytosis and endocytosis: modes, functions, and coupling mechanisms. Annual Review of Physiology 76, 301-331.

Yang, W. Q., Braun, C., Plattner, H., Purvee, J. \& Van Houten, J. L. (1997). Cyclic nucleotides in glutamate chemosensory signal transduction of Paramecium. Journal of Cell Science 110, 2567-2572.

Yano, J., Rachochy, V. \& Van Houten, J. L. (2003). Glycosyl phosphatidylinositol-anchored proteins in chemosensory signaling: antisense manipulation of Paramecium tetraurelia PIG-A gene expression. Eukaryotic Cell 2, 1211-1219.

Yano, J., Rajendran, A., Valentine, M. S., Saha, M., Ballif, B. A. \& Van Houten, J. L. (2013). Proteomic analysis of the cilia membrane of Paramecium tetraurelia. Fournal of Proteomics 78, 113-122.

Yildiz, M. T. \& ArsLanyolu, M. (2014). In silico identification and characterization of the MAPK family members of unicellular model eukaryote Tetrahymena thermophila. European Fournal of Protistology 50, 538-550.

Ying, G., Avasthi, P., Irwin, M., Gerstner, C. D., Frederick, J. M., Lucero, M. T. \& BAEHR, W. (2014). Centrin 2 is required for mouse olfactory ciliary trafficking and development of ependymal cilia planar polarity. Fournal of Neuroscience 34, 6377-6388.

ZACKrofF, R. V. \& HufNagel, L. A. (2002). Induction of anti-actin drug resistance in Tetrahymena. Foumal of Eukaryotic Microbiology 49, 475-477.

Zampese, E. \& Pizzo, P. (2012). Intracellular organelles in the saga of $\mathrm{Ca}^{2+}$ homeostasis: different molecules for different purposes? Cellular and Molecular Life Sciences 69, 1077-1104.

Zerial, M. \& McBride, H. (2001). Rab proteins as membrane organizers. Nature Revieves Molecular Cell Biology 2, 107-117.

Zhang, C., GaO, S., Molascon, A. J., Wang, Z., Gorovsky, M. A., Liu, Y. \& ANDREws, P. C. (2014). Bioinformatic and proteomic analysis of bulk histones reveals PTM crosstalk and chromatin features. Fournal of Proteome Research 13, 3330-3337.

Zieseniss, E. \& Plattner, H. (1985). Synchronous exocytosis in Paramecium cells involves very rapid $(\leq 1 \mathrm{~s})$, reversible dephosphorylation of a $65-\mathrm{kD}$ phosphoprotein in exocytosis-competent strains. Fournal of Cell Biology 101, 2028-2035.

Zito, K. \& Scheuss, V. (2009). NMDA receptor function and physiological modulation. In Encyclopedia of Neuroscience (Volume 6, ed. L. R. SQUiRE), pp. 1157-1164. Academic Press, Oxford.

Zmasek, C. M. \& Godzik, A. (2013). Evolution of the animal apoptois network. Cold Spring Harbor Perspectives in Biology 5, a008649.

Zweifel, E., Smith, J., Romero, D., Giddings, T. H., Winey, M., Honts, J., Dahlseid, J., Schneider, B. \& Cole, E. S. (2009). Nested genes CDA12 and CDA13 encode proteins associated with membrane trafficking in the ciliate Tetrahymena thermophila. Eukaryotic Cell 8, 899-912. 


\section{APPENDIX:}

\section{BIOLOGIGAL AND TEGHNIGAL TERMS: GLOS- SARY, AND ABBREVIATIONS USED}

AAA-ATPase: triple-A ATPase: a chaperone (protein mediating correct conformation of another protein). For example NSF is a chaperone specific for SNARE proteins, occurring in protozoa, animals and plants; ADF: actin depolymerising factor; AED: aminoethyldextrane: secretagogue stimulating trichocyst exocytosis in Paramecium spp; Alveolar sacs: flat, sac-like cortical $\mathrm{Ca}^{2+}$ stores closely attached to the cell membrane in ciliates and other Alveolata; AP: adaptor protein: APs mediate self-assembly of coat proteins on vesicles budding from the cell membrane or from specific organelles. Found in protozoa, animals and plants; ARNO: ADP [adenosine diphosphate]-ribosylation factor nucleotide site opener, a guanosine nucleotide exchange factor; ASI2: signal transducer and activator of transcription 2 protein; BAR: Bin-amphiphysin-Rvs; bbof1: basal body orientation factor 1; BBS: Beadle-Bardet Syndrome: molecular defect of ciliary function in man, with an ortholog of BBS-protein in ciliates; $\mathbf{C} 2$ domain: protein domain with B-barrel conformational motif and an extending loop appropriate for low-capacity/high-sensitivity $\mathrm{Ca}^{2+}$ binding. Found in protozoa, animals and plants; $\left[\mathbf{C a}^{2+}\right]_{\mathbf{i}}$ : intracellular free (dissolved, not protein-bound) $\mathrm{Ca}^{2+}$ concentration; $\left[\mathbf{C a}^{2+}\right]_{0}$ : extracellular $\mathrm{Ca}^{2+}$ concentration; $\mathrm{Ca}^{2+}$-ATPase: $\mathrm{Ca}^{2+}$ pump for primary active $\mathrm{Ca}^{2+}$ transport, forming a phosphorylated intermediate (in contrast to $\mathrm{V}-\mathrm{H}^{+}$-ATPase). Found in protozoa, animals and plants; cADPR: cyclic adenosine diphosphoribose. Second messenger; cAMP: cyclic adenosine monophosphate (AMP); CaBP: $\mathrm{Ca}^{2+}$-binding protein: high capacity/low affinity (in cytosol and $\mathrm{Ca}^{2+}$ storage organelles) or low capacity/high affinity (in cytosol). Found in all kingdoms; CaM: calmodulin: $\mathrm{Ca}^{2+}$-binding protein with four EF-hand motifs (each usually made of 12 amino acids) enabling $\mathrm{Ca}^{2+}$ binding with low capacity/high affinity. Found in all kingdoms; CaM-BP: calmodulin-binding protein; CDA11, 12: cell division (protein) Al1, 12; Cdk: cyclin-dependent kinase; GDPK: $\mathrm{Ca}^{2+}$-dependent protein kinase: a protein kinase with integrated calmodulin-like motif, specific to plants and some protozoa such as ciliates; Contractile vacuole complex: osmoregulatory organelle with rhythmic pulsations, serving for the extrusion of an excess of water (and ions) in some freshwater protozoa; COP: coatamer protein: ubiquitous non-clathrin-type polymeric protein assembly mediating vesicle budding, e.g. from Golgi apparatus; CRC: $\mathrm{Ca}^{2+}$-release channel: see $\mathrm{IP}_{3} \mathrm{R}, \mathrm{RyR}$ and RyR-LP; recently identified in protozoa (ciliates and trypanosomatids), but absent from plants; DAG: diacyl glycerol: protein kinase C-activating product formed (together with inositol 1,4,5-trisphosphate) by phospholipase C-mediated cleavage of phosphatidyl inositol 4,5-bisphosphate; occurrence not established for ciliates; DIE: defective in excision; DisAp: disorientation protein mutant A; DOG2: double C2-like domain containing protein 2; EDX: energy-dispersive X-ray microanalysis: electron microscopic analysis of local element composition by element-specific X-ray fluorescence generated by the electron beam used for image formation. Chemical signals can be assigned to specific subcellular structures, such as $\mathrm{Ca}^{2+}$ stores. The method can be applied to cells rapidly frozen under defined functional states (quenched-flow/rapid freezing). EDX allows for quantitation, e.g. measuring changes in total $\mathrm{Ca}^{2+}$ content in an organelle during stimulation; EEA1: early endosome antigen 1; EF-hand motif: helix-loop-helix motif of CaBPs for high affinity $\mathrm{Ca}^{2+}$ binding; EMRE: essential MCU regulator; ER: endoplasmic reticulum; Er-1: Euplotes raikovi pheromone; Erk: extracellular signal-regulated kinase; FIPs: family of RabII-interacting proteins; fMLP: formyl-methionyl-leucyl-phenylalanine; FOR20: FOP (FGFR1 oncogene partner)-related protein of $20 \mathrm{kDa}$ size; GAP: GTPase-activating protein: activator of small (monomeric) GTPases ('G-proteins'); GDI: guanosine nucleotide dissociation inhibitor: inhibits GTP/GDP exchange on small GTPases; GEF: guanosine nucleotide exchange factor: stimulates GTP/GDP exchange on small GTPases, i.e. monomeric guanosine trisphosphate (GTP) binding proteins with GTPase (GTP hydrolysing) function; GFP: green fluorescent protein; GPCR: G-protein-coupled receptor: receptors mainly on cell surface coupled to trimeric G-proteins (with $\alpha$-, $\beta$ - and $\gamma$-subunits); occurrence in ciliates likely, but not definitely established; GPI-anchor: glycosyl phosphatidylinositol-anchor: attachment of a protein to a membrane via a carboxyterminal anchor formed by glycosyl residues complemented by a phosphate and an inositol group. Found in protozoa, animals and plants; G-proteins: proteins binding guanosine nucleotides. Specifically GTPase molecules [small (monomeric) G-proteins involved, e.g. in vesicle trafficking] and trimeric G-proteins (involved in signalling from the cell membrane); Grlp: granule lattice protein; Grtp: granule tip protein; GTPase: small monomeric G-proteins involved, e.g. in vesicle trafficking. Found in protozoa, animals and plants; HAP2: a transcriptional activator; IES: internal eliminated sequence: defined stretches of micronuclear DNA (probably derived from old transposons) occurring in ciliates; they are eliminated under epigenetic control, i.e. by scanning RNA (scnRNA) during formation of a new macronuclear genome from a postmitotic micronuclear genome; IFT: intraflagellar transport: this also includes intraciliary transport of macromolecule aggregates; in protozoa and animals; ILF: infraciliary lattice filaments; $\mathbf{I n s} \mathbf{P}_{3}$ : inositol 1,4,5-trisphosphate $\left(\operatorname{Ins}_{3}\right)$ : formed (together with diacylglycerol) by phospholipase C-mediated cleavage of phosphatidyl inositol 4,5-bisphosphate; activates $\mathrm{InsP}_{3}$ receptors (CRCs). $\mathrm{InsP}_{3}$ effects reported from all kingdoms, but formation in plants is unlikely; $\mathbf{I P}_{3} \mathbf{R}$ : inositol 1,4,5-trisphosphate receptor: $\mathrm{Ins}_{3}$-receptor-type CRCs; established from protozoa onwards, including ciliates and trypanosomatids, and animals; expected also for choanoflagellates; kif: kinesin family; -LP: -like protein: expressing similarity, e.g. between calmodulin and calmodulin-like sequences in some proteins; MALDI: matrix-assisted laser desorption/ionisation: mass spectrometric analysis of 
biomolecules, based on binding to a support matrix, laser irradiation for fragmentation and desorption from the matrix and ionisation, coupled to acceleration of ionised fragments for mass recording in a spectrometer. Phosphorylation sites in proteins can thus be determined; MAPK: mitogen-activated protein kinase: protein kinase activated during mitosis and some other functions; probably in all eukaryotic kingdoms, but little studied in ciliates; MGU: mitochondrial calcium uniporter; MEK: MAP and ERK kinase $(\mathrm{ERK}=$ extracellular-signal regulated kinase): protein kinase involved in intracellular signalling after MAPK activation; probably in all eukaryotic kingdoms, but little studied in ciliates; MICU: mitochondrial calcium uptake; MOB1: Mps One Binder; mtA: name of a promotor; Mucocysts: dense core-secretory organelles of Tetrahymena spp; Myo: myosin; NAADP: nicotinic acid adenine dinucleotidephosphate. Second messenger; NFAT: nuclear factor for activation of T-cells; NHEJ: non-homologous end-joining; NIMA: never in mitosis A; NMDA: N-methyl-D-aspartate; NSF: N-ethylmaleimide sensitive factor: SNARE-specific chaperone (see AAA-ATPase) serving for proper conformational arrangement of SNARE proteins. Found in all kingdoms; Pdsg: Paramecium protein involved in development of the somatic genome; PIns $\mathbf{P}_{2}$ : phosphatidylinositol 4,5-bisphosphate; PKA: cAMP-activated protein kinase A: activated by cyclic $3^{\prime}, 5^{\prime}$-adenosinemonophosphate. Found in all kingdoms; PKC: protein kinase $\mathrm{C}$ : activated by diacyl glycerol; present in animals, but not identified at a molecular level in plants, ciliates and other protozoa; PKG: cGMP-activated protein kinase: activated by cyclic $3^{\prime}, 5^{\prime}$-guanosinemonophosphate. Found in all kingdoms; PLG: phospholipase C; PMCA: plasma membrane $\mathrm{Ca}^{2+}$-ATPase: $\mathrm{Ca}^{2+}$ pump in the cell membrane. Found in all kingdoms; PP1: protein phosphatase 1; PP2B: protein phosphatase 2B: dimeric protein identical with calcineurin. Described in Dictyostelium and Paramecium spp., also occurs in animals whereas in plants only subunit $\mathrm{B}$ is found; PrP: prion protein; PtAct, PtCRC, PtSyb, PtSyx, PtSNARE etc.: $P t$ designates proteins from $P$. tetraurelia, such as actin etc. Subfamilies are indicated with roman numbers and individual paralogs (including ohnologs) by Arabic numbers; e.g. PtCRC-II-1 and -2 indicate specific $\mathrm{Ca}^{2+}$-release channels; Rab: 'Ras-related in brain': small GTPase family invoved in directing intracellular vesicle traffic in all kingdoms; Rho: $\rho$ factor, a GTPase; RyR: ryanodine receptor: type of $\mathrm{Ca}^{2+}$-release channel previously characterised by its response to the plant toxin ryanodine; typical for sarcoplasmic reticulum in muscle cells, but also occurring in many other animal cells. Not established in plant cells. A prototype occurs in ciliates; RyR-LP: ryanodine receptor-like protein: a ryanodine receptor-type $\mathrm{Ca}^{2+}$-release channel found in Paramecium spp; scnRNA: scanning RNA: recognises internal eliminated sequences (IES), i.e. defined stretches of micronuclear DNA (probably derived from old transposons) in ciliates, to be eliminated under epigenetic control during formation of a new macronuclear genome; Sec7: transport protein 7: a guanine nucleotide exchange factor, relevant for secretion; SERCA: sarcoplasmic/endoplasmic reticulum $\mathrm{Ca}^{2+}$-ATPase: $\mathrm{Ca}^{2+}$ pump in the sarcoplasmic reticulum of muscle cells and in the endoplasmic reticulum of protozoa, plants and animals; SH2: Src homology domain 2; SNAP-25: synaptosomal associated protein of $25 \mathrm{kDa}$ size; SNAREs: soluble N-ethylmaleimide sensitive factor (NSF) attachment protein receptors: single-span membrane proteins of specific types distributed over membranes undergoing interaction (docking) for final fusion; see scheme in Fig. 1. The differentiation between $t-S N A R E s(t=$ target $)$ and $\mathrm{v}$-SNAREs $(\mathrm{v}=$ vesicle) may not reflect the real topology. Therefore, alternative designations are Q-SNAREs and R-SNARE, for t- and v-SNAREs, respectively, according to the predominant 'central' amino acid in the SNARE domain. Found in all kingdoms; $\mathbf{S O C}(\mathbf{E})$ : store-operated $\mathrm{Ca}^{2+}$ (entry): also called capacitative $\mathrm{Ca}^{2+}$ influx. SOC (E) superimposes mobilisation of $\mathrm{Ca}^{2+}$ from a cortical store as a first step. Found from Paramecium to man, but probably not in plants; SPFH: stomatin, prohibitin, flotillin and HflK/C superfamily; Spt6: transcription activation factor; Src: name derived from sarcoma; a non-receptor tyrosine kinase family; Stat: transcription activation factor; SUMO: small ubiquitin-like modifier; Syb: synaptobrevin: v-/R-SNARE (see SNARE), e.g. on trafficking vesicles. Universal distribution, although as 'longin' forms in ciliates and plants; Syx: syntaxin: t-/Q-SNARE (see SNARE), e.g. at the cell membrane and on trafficking vesicles. Found in all kingdoms; TMN1: Tetrahymena mitochondrial nuclease; tmp: trichocyst matrix protein; TPG: two-pore channel: cation $\left(\mathrm{Ca}^{2+}\right)$ channels with dimeric design of a split voltage-gated $\mathrm{Ca}^{2+} / \mathrm{Na}^{+}$ channel, typically occurring in acidic compartments. Found in acidic compartments of plants and animals, whereas only indirect evidence is available from ciliates; Trichocysts: dense core-secretory organelles of Paramecium spp; TRP: transient receptor potential channels; t-SNARE/Q-SNAREs: see SNAREs; VPS: vacuolar protein sorting protein; vsAG: variant surface antigens: GPI-anchored glycoprotein components of the ciliate cell surface, with mutually exclusive expression under epigenetic control. Found in all kingdoms; v-SNARE/R-SNARE: see SNAREs. 